\title{
Partial Identification of Economic Mobility: With an Application to the United States
}

\author{
Daniel L. Millimet \\ Southern Methodist University \& IZA \\ Hao $\mathrm{Li}^{*}$ \\ Nanjing Audit University \\ Punarjit Roychowdhury \\ Indian Institute of Management, Indore
}

January 3, 2019

\begin{abstract}
The economic mobility of individuals and households is of fundamental interest. While many measures of economic mobility exist, reliance on transition matrices remains pervasive due to simplicity and ease of interpretation. However, estimation of transition matrices is complicated by the well-acknowledged problem of measurement error in self-reported and even administrative data. Existing methods of addressing measurement error are complex, rely on numerous strong assumptions, and often require data from more than two periods. In this paper, we investigate what can be learned about economic mobility as measured via transition matrices while formally accounting for measurement error in a reasonably transparent manner. To do so, we develop a nonparametric partial identification approach to bound transition probabilities under various assumptions on the measurement error and mobility processes. This approach is applied to panel data from the United States to explore short-run mobility before and after the Great Recession.
\end{abstract}

JEL: C18, D31, I32

Keywords: Partial Identification, Measurement Error, Mobility, Transition Matrices, Poverty

*The authors are grateful for helpful comments from the editor, Rajeev Dehejia, two anonymous referees, Hao Dong, Elira Kuka, Essie Maasoumi, Xun Tang, and conference participants at Texas Econometrics Camp XXII and the LACEA-LAMES 2018 Annual Meeting. Corresponding author: Hao Li, Department of Economics, Nanjing Audit University, Nanjing, Jiangsu, China. E-mail: lihao@nau.edu.cn. 


\section{Introduction}

There has been substantial interest of late in intra- and inter-generational mobility. Dang et al. (2014, p. 112) state that mobility "is currently at the forefront of policy debates around the world." Within the popular press, it has been noted that "social mobility ... has become a major focus of political discussion, academic research and popular outrage in the years since the global financial crisis."11 In this paper, we study economic mobility while accounting for measurement error in income data. Specifically, we offer a new approach to addressing measurement error in the estimation of transition matrices.

Measurement error in income data is known to be pervasive, even in administrative data. In survey data, measurement error arises for two main reasons: misreporting (particularly with retrospective data) and imputation of missing data (Jäntti and Jenkins 2015). It is now taken as given that self-reported income in survey data contain significant measurement error, and that the measurement error is nonclassical in the sense that it is mean-reverting and serially correlated (Bound et al. 2001; Kapteyn and Ypma 2007; Gottschalk and Huynh 2010). Compounding matters, Meyer et al. (2015) find that both problems - nonresponse and accuracy conditional on answering - are worsening over time. In administrative data, measurement error arises for three main reasons: misreporting (tax evasion or filing errors), conceptual differences between the desired and available income measures, and processing errors (Bound et al. 2001; Kapteyn and Ypma 2007; Pavlopoulos et al. 2012; Meyer et al. 2015). Even if administrative data are entirely accurate, they are only available in a handful of developed countries.

However, existing studies of mobility either ignore the issue or utilize complex solutions that invoke strong (and often non-transparent) identification assumptions and have data requirements that are quite limiting. The most frequent response to measurement error in the empirical literature on mobility is to mention it as a caveat (Dragoset and Fields 2006). While the usual assumption is that measurement error will bias measures of mobility upward, the complexity of mobility measures along with the nonclassical nature of the measurement

\footnotetext{
${ }^{1}$ See Washington Post (October 6, 2016) at https://www.washingtonpost.com/news/wonk/wp/ 2016/10/06/striking-new-research-on-inequality-whatever-you-thought-its-worse/?utm_term= $.83 d 37 c 53195 b$.
} 
error makes the direction of any bias uncertain. Glewwe (2012, p. 239) states that "all indices of relative mobility tend to exaggerate mobility if income is measured with error," yet others offer a different opinion. Dragoset and Fields (2006, p. 1) contend that "very little is known about the degree to which earnings mobility estimates are affected by measurement error." Gottschalk and Huynh (2010, p. 302) note that "the impact of nonclassical measurement error on mobility is less clear since mobility measures are based on the joint distribution of reported earnings in two periods."

Our approach to the analysis of mobility given measurement error in income data concentrates on the partial identification of transition matrices. We provide informative bounds on the transition probabilities under minimal assumptions concerning the measurement error process and a variety of nonparametric assumptions on income dynamics. To our knowledge, this is the first study to extend the literature on partial identification to the study of transition matrices (see, e.g., Horowitz and Manski 1995; Manski and Pepper 2000) ${ }^{2}$ Within this environment, we first derive sharp bounds on transition probabilities under minimal assumptions on the measurement error process. We then show how the bounds may be narrowed by imposing more structure via shape restrictions, level set restrictions that relate transition probabilities across observations with different attributes (Manski 1990; Lechner 1999), and monotonicity restrictions that assume monotonic relationships between the true income and certain observed covariates (Manski and Pepper 2000).

In contrast to existing approaches to address measurement error in studies of mobility (discussed in Section 2), our approach has several distinct advantages. First, the assumptions invoked to obtain a given set of the bounds are transparent, easily understood by a wide audience, and easy to impose or not impose depending on the particular context. Moreover, bounds on the elements of transition matrices extend naturally to bounds on mobility measures derived from transition matrices. Second, our approach only requires data at two points in time. Third, our approach is easy to implement (through our creation of a generic

\footnotetext{
${ }^{2}$ In closely related work, Vikström et al. (2018) study the partial identification of treatment effects where the outcomes are conditional transition probabilities. In their setup, measurement error is not considered. Rather, point identification fails even under randomized treatment assignment as treatment assignment is not guaranteed to be independent of potential outcomes in future periods conditional on intermediate outcomes. Our approach is also similar to Molinari (2008); she studies the partial identification of the distribution of a discrete variable that is observed with error.
} 
Stata command) $!^{3}$ Fourth, our approach extends easily to applications other than income, such as dynamics related to consumption, wealth, occupational status, labor force status, health, student achievement, etc.

The primary drawback to our approach is the lack of point identification. Two responses are in order. First, our approach should be viewed as a complement to, not a replacement for, existing approaches. Indeed, one usefulness of our approach is to provide bounds with which point estimates derived via alternative estimation techniques may be compared. Second, many existing approaches to deal with measurement error in mobility studies end up producing bounds even though the solutions are not couched as a partial identification approach (e.g., Dang et al. 2014; Lee et al. 2017). This arises due to an inability to identify all parameters in some structural model of observed and actual incomes.

Perhaps a secondary drawback of our approach is the focus on transition matrices to capture mobility. Such matrices have the disadvantage of not providing a scalar measure of mobility, simplifying spatial and temporal comparisons of mobility. While there is merit to this critique, there are several responses. First, transition matrices are an obvious starting point in the measurement of mobility. Jäntti and Jenkins (2015, p. 822) argue that, when measuring mobility across two points in time, "the bivariate joint distribution of income contains all the information there is about mobility, so a natural way to begin is by summarizing the joint distribution in tabular or graphical form." Second, transition matrices are easily understood by policymakers and the general public and thus are frequently referenced within these domains. Third, transition matrices allow one to examine mobility at different parts of the income distribution (Lee et al. 2017). Finally, bounds on (scalar) measures of mobility derived from the elements of transition matrices are easily obtained from our approach.

We illustrate our approach with an examination of intragenerational mobility in the United States using data from the Survey of Income and Program Participation (SIPP). Specifically, we examine mobility over two four-year periods, 2004 to 2008 and 2008 to 2012. Understanding mobility patterns in the US is important as there is convincing evidence that income inequality has been increasing in the US.4 However, the welfare impact of this

\footnotetext{
${ }^{3}$ Available at http://faculty.smu.edu/millimet/code.html

${ }^{4}$ The level of income inequality in the US has followed a U-shaped pattern over the past century (Picketty and Saez 2003; Kopczuk et al. 2010; Atkinson and Bourguignon 2015).
} 
rise depends crucially on the level of economic mobility. Shorrocks (1978, p. 1013) argues that "evidence on inequality of incomes or wealth cannot be satisfactorily evaluated without knowing, for example, how many of the less affluent will move up the distribution later in life." More recently, Kopczuk et al. (2010, p. 91-2) conclude that "a comprehensive analysis of disparity requires studying both inequality and mobility" as "annual earnings inequality might substantially exaggerate the extent of true economic disparity among individuals."

Our analysis of US mobility yields some striking results. First, we show that relatively small amounts of measurement error leads to bounds that can be quite wide in the absence of other information or restrictions. Second, the restrictions considered contain significant identifying power as the bounds can be severely narrowed. Third, allowing for misclassification errors in up to $10 \%$ of the sample, we find that the probability of being in (out of) poverty in 2008 conditional on being in poverty in 2004 is at least $35 \%$ (27\%) under our most restrictive set of assumptions. The probability of being in (out of) poverty in 2012 conditional on being in poverty in 2008 is at least $36 \%(25 \%)$ under our most restrictive set of assumptions. Finally, the probability of being in poverty in 2008 conditional on not being in poverty in 2004 is at least $2 \%$ and no more than $11 \%$ under our most restrictive set of assumptions. The probability of being in poverty in 2012 conditional on not being in poverty in 2008 is at least $4 \%$ and no more than $13 \%$ under our most restrictive set of assumptions.

The rest of the paper is organized as follows. Section 2 provides a brief review of existing approaches to address measurement error in studies of mobility. Section 3 presents our partial identification approach. Section 4 contains the empirical application. Section 5 concludes.

\section{Literature Review}

Burkhauser and Couch (2009) and Jäntti and Jenkins (2015) provide excellent reviews of the numerous mobility measures. Bound et al. (2001) and Meyer et al. (2015) offer excellent surveys regarding measurement error in microeconomic data. Tamer (2010), Bontemps and Magnac (2018), and Ho and Rosen (2017) provide in depth reviews of the recent literature on partial identification $5^{5}$ Here, we focus on approaches that have been taken to address (or

\footnotetext{
${ }^{5}$ Within the partial identification literature, our analysis is most closely related to Molinari (2008), who posits a direct misclassification approach in order to bound the distribution of a discrete variable in the
} 
not address) measurement error in analyses of economic mobility. We identify three general approaches in the existing literature: (i) ignore it, (ii) ad hoc data approaches, and (iii) structural approaches. In the interest of brevity, we relegate much of the discussion of the prior literature to Appendix A. Here, we discuss only those methods most comparable to our approach. These methods fall within the third category and utilize structural models to simulate error-free income. Armed with the simulated data, any mobility measure may be computed, including transition matrices. Clearly, the validity of this approach rests on the quality of the simulated error-free data. Obtaining simulated values of error-free data is not trivial and typically relies on complex models invoking a number of fairly opaque assumptions.

Studies pursuing this strategy include McGarry (1995), Glewwe and Dang (2011), Pavlopoulos et al. (2012), Dang et al. (2014), and Lee et al. (2017). McGarry (1995) posits a variance components model to isolate the portion of observed income that represents measurement error. Upon simulating error-free income, conditional staying probabilities for the poor are examined. The results indicate substantially less mobility in the simulated data. However, the model defines measurement error as the individual-level, time-varying, serially uncorrelated component of income. Thus, all time-varying idiosyncratic sources of income variation are removed. Moreover, the individual-level, time-varying, serially correlated component of income is not considered measurement error. Finally, parametric distributional assumptions are required for identification in practice.

Glewwe and Dang (2011) begin with the assumption that log income follows an AR(1) process. The authors then combine OLS and IV estimates of the forward and reverse regressions, along with assumptions about the variance components of the model, to simulate error-free income. The simulated data are then used to assess income growth across the distribution. As in McGarry (1995), the results suggest substantial bias from measurement error. However, as in McGarry (1995), identification of error-free income relies on strong assumptions for identification, such as serially uncorrelated measurement error, particular functional forms, and valid instrumental variables.

presence of misclassification errors, and studies of partial identification of treament effects under nonrandom selection and misclassification of treatment assignment (e.g., Kreider and Pepper 2007, 2008; Gundersen and Kreider 2008, 2009; Kreider et al. 2012). 
Pavlopoulos et al. (2012) build on Rendtel et al. (1998) and specify a mixed latent Markov model to examine error-free transitions between low pay, high pay, and nonemployment. The model requires data from at least three periods, as well as requires perhaps strong assumptions concerning unobserved heterogeneity and initial conditions. In addition, serial correlation in measurement error is difficult to address and extending the model to more than three states is problematic. Nonetheless, the results align with the preceding studies in that mobility is dampened once measurement error is addressed.

Dang et al. (2014) consider the measurement of mobility using pseudo-panel data. Since the same individuals are not observed in multiple periods, the authors posit a static model of income using only time invariant covariates available in all periods. The model estimates, along with various assumptions concerning how unobserved determinants of income are correlated over time, are used to bound measures of a two-by-two poverty transition matrix. This approach implicitly addresses measurement error through the imputation process as missing data can be considered an extreme form of measurement error. However, measurement error in observed incomes used to estimate the static model and compute the poverty transition matrix is not addressed. Moreover, it is not clear how one could extend the method to estimate more disaggregate transition matrices.

Finally, Lee et al. (2017) estimates a complex model based on an AR(1) model of consumption dynamics with time invariant and time-varying sources of measurement error to simulate error-free consumption and estimate transition matrices. Consistent with the preceding studies, significantly less mobility is found in the simulated data. While the authors' model has some advantages compared to earlier attempts to simulate error-free outcomes, these advantages come at a cost of increased complexity, decreased transparency of the identifying assumptions, and a need for four periods of data. In addition, bounds are obtained as not all parameters required for the simulations are identified.

In summary, the literature on addressing measurement error in studies of mobility has witnessed significant recent growth. However, there remains much scope for additional work. While simulation-based methods allow for estimation of transition matrices, these methods are complex, lack transparency, rely on strong functional form and distributional assumptions, and often require more than two years of data. Moreover, the common reliance in 
the majority of the simulation approaches on an $\mathrm{AR}(1)$ model of income or consumption dynamics is worrisome. Lee et al. (2017, p. 38) acknowledge that "this model is not so much derived from a well-developed theory, but it is a convenient reduced-form model." Finally, the reliance on precise assumptions concerning the nature of the variance components is unappealing in light of Kapteyn and Ympa's (2007, p. 535) finding that "substantive conclusions may be affected quite a bit by changes in assumptions on the nature of error in survey and administrative data."

Our proposed approach complements these existing approaches. However, in contrast to simulation approaches, which often end up with bounds on transition probabilities, we set out to estimate bounds from the beginning, making it transparent exactly how the bounds are affected by each assumption one may wish to impose. Furthermore, the assumptions imposed to narrow the bounds are optional and much easier for non-experts to comprehend.

\section{Model}

\subsection{Setup}

Let $y_{i t}^{*}$, denote the true income for observation $i, i=1, \ldots, N$, in period $t, t=0,1$. An observation may refer to an individual or household observed at two points in time in the case of intragenerational mobility or a parent-child pair observed at two points in time in the case of intergenerational mobility. Further, let $F_{0,1}\left(y_{0}^{*}, y_{1}^{*}\right)$ denote the joint (bivariate) cumulative distribution function $(\mathrm{CDF})$, where $y_{t}^{*} \equiv\left[y_{1 t}^{*} \cdots y_{N t}^{*}\right]$.

While movement through the distribution from an initial period, 0, to a subsequent period, 1 , is completely captured by $F_{0,1}\left(y_{0}^{*}, y_{1}^{*}\right)$, this is not practical. A $K \times K$ transition matrix, $P_{0,1}^{*}$, summarizes this joint distribution and is given by

$$
P_{0,1}^{*}=\left[\begin{array}{cccc}
p_{11}^{*} & \cdots & \cdots & p_{1 K}^{*} \\
\vdots & \ddots & & \vdots \\
\vdots & & \ddots & \vdots \\
p_{K 1}^{*} & \cdots & \cdots & p_{K K}^{*}
\end{array}\right]
$$


Elements of this matrix have the following form

$$
\begin{aligned}
p_{k l}^{*} & =\frac{\operatorname{Pr}\left(\zeta_{k-1}^{0} \leq y_{0}^{*}<\zeta_{k}^{0}, \zeta_{l-1}^{1} \leq y_{1}^{*}<\zeta_{l}^{1}\right)}{\operatorname{Pr}\left(\zeta_{k-1}^{0} \leq y_{0}^{*}<\zeta_{k}^{0}\right)} \\
& =\frac{\operatorname{Pr}\left(y_{0}^{*} \in k, y_{1}^{*} \in l\right)}{\operatorname{Pr}\left(y_{0}^{*} \in k\right)} \quad k, l=1, \ldots, K,
\end{aligned}
$$

where the $\zeta$ s are cutoff points between the $K$ partitions such that $0=\zeta_{0}^{t}<\zeta_{1}^{t}<\zeta_{2}^{t}<$ $\left.\cdots<\zeta_{K-1}^{t}<\zeta_{K}^{t}<\infty, t=0,1\right]$ Thus, $p_{k l}^{*}$ is a conditional probability. A complete lack of mobility implies $p_{k l}^{*}$ equals unity if $k=l$ and zero otherwise..$^{7}$ Finally, we can define conditional transition matrices, conditioned upon $X=x$, where $X$ denotes a vector of observed attributes. Denote the conditional transition matrix as $P_{0,1}^{*}(x)$, with elements given by

$$
\begin{aligned}
p_{k l}^{*}(x) & =\frac{\operatorname{Pr}\left(\zeta_{k-1}^{0} \leq y_{0}^{*}<\zeta_{k}^{0}, \zeta_{l-1}^{1} \leq y_{1}^{*}<\zeta_{l}^{1} \mid X=x\right)}{\operatorname{Pr}\left(\zeta_{k-1}^{0} \leq y_{0}^{*}<\zeta_{k}^{0} \mid X=x\right)} \\
& =\frac{\operatorname{Pr}\left(y_{0}^{*} \in k, y_{1}^{*} \in l \mid X=x\right)}{\operatorname{Pr}\left(y_{0}^{*} \in k \mid X=x\right)} \quad k, l=1, \ldots, K .
\end{aligned}
$$

Implicit in this definition is the assumption that $X$ includes only time invariant attributes $:^{8}$

For clarity, throughout the paper we consider two types of transition matrices: (i) those with equal-sized partitions and (ii) those with unequal-sized partitions. With equal-sized partitions, the $\zeta$ s are chosen such that each partition contains $1 / K$ of the population. For example, equal-sized partitions with $K=5$ correspond to a quintile transition matrix. In this case, the rows and columns of $P_{0,1}^{*}$ sum to one and mobility is necessarily zero-sum (i.e., if an observation is misclassified in the upward direction, there must be at least one observation misclassified in the downward direction). With unequal-sized partitions, only the rows of $P_{0,1}^{*}$ sum to one and mobility is not zero-sum. For example, we shall consider the case of a $2 \times 2$ poverty transition matrix, where $\zeta_{1}^{t}$ is the poverty line in period $t$.

\footnotetext{
${ }^{6}$ For example, if $K=5$, then the cutoff points might correspond to quintiles within the two marginal distributions of $y_{0}^{*}$ and $y_{1}^{*}$.

${ }^{7}$ In contrast, 'perfect' mobility may be characterized by origin-destination independence, implying $p_{k l}^{*}=$ $1 / K$ for all $k, l$, or by complete rank reversal, implying $p_{k l}^{*}=1$ if $k+l=K+1$ and zero otherwise. See Jäntti and Jenkins (2015) for discussion.

${ }^{8}$ Note, while the probabilities are conditional on $X$, the cutoff points $\zeta$ are not. Thus, we are capturing movements within the overall distribution among those with $X=x$.
} 
Given the definition of $P_{0,1}^{*}$ or $P_{0,1}^{*}(x)$, our objective is to learn something about its elements. With a random sample $\left\{y_{i t}^{*}, x_{i}\right\}$ and a choice of $K$ and the $\zeta$ s, the transition probabilities are point identified as they are functions of nonparametrically estimable quantities. The corresponding plug-in estimator is consistent. However, as stated previously, ample evidence indicates that income is measured with error. Let $y_{i t}$ denote the observed income for observation $i$ in period $t$. With data $\left\{y_{i t}, x_{i}\right\}$ and a choice of $K$ and the $\zeta$ s, the empirical transition probabilities are inconsistent for $p_{k l}^{*}$ and $p_{k l}^{*}(x)$.

With access only to data containing measurement error, our goal is to bound the probabilities given in (2) and (3). The relationships between the true partitions of $\left\{y_{i t}^{*}\right\}_{t=0}^{1}$ and the observed partitions of $\left\{y_{i t}\right\}_{t=0}^{1}$ are characterized by the following joint probabilities:

$$
\theta_{(k, l)}^{\left(k^{\prime}-k, l^{\prime}-l\right)}=\operatorname{Pr}\left(y_{0} \in k^{\prime}, y_{1} \in l^{\prime}, y_{0}^{*} \in k, y_{1}^{*} \in l\right)
$$

While conditional misclassification probabilities are more intuitive, these joint probabilities are easier to work with (e.g., Kreider et al. 2012).

In (4) the subscript $(k, l)$ indexes the true partitions in period 0 and 1 and the superscript $\left(k^{\prime}-k, l^{\prime}-l\right)$ indicates the degree of misclassification given by the differences between the observed partitions $k^{\prime}$ and $l^{\prime}$ and true partitions $k$ and $l$. If $k^{\prime}-k, l^{\prime}-l>0$, then there is upward misclassification in both periods. If $k^{\prime}-k, l^{\prime}-l<0$, then there is downward misclassification in both periods. If $k^{\prime}-k$ and $l^{\prime}-l$ are of different signs, then the direction of misclassification changes across periods. $\theta_{(k, l)}^{(0,0)}$ represents the probability of no misclassification in either period for an observation with true income in partitions $k$ and $l ! 9$

\footnotetext{
${ }^{9} \theta_{(k, l)}^{(0,0)}$ may be strictly positive even though income is misreported in either or both periods (i.e., $y_{i t} \neq y_{i t}^{*}$ for at least some $i$ and $t$ ) as long as the misreporting is not so severe as to invalidate the observed partitions (i.e., $k^{\prime}=k$ and $l^{\prime}=l$ regardless). Throughout the paper, we use the term measurement error to refer to errors in observed income $\left(y_{i t} \neq y_{i t}^{*}\right)$ and misclassification to refer to errors in the observed partitions $\left(k^{\prime} \neq k\right.$ and/or $l^{\prime} \neq l$ ).
} 
With this notation, we can now rewrite the elements of $P_{0,1}^{*}$ as

$$
\begin{aligned}
& p_{k l}^{*}=\frac{\operatorname{Pr}\left(y_{0}^{*} \in k, y_{1}^{*} \in l\right)}{\operatorname{Pr}\left(y_{0}^{*} \in k\right)} \\
& \operatorname{Pr}\left(y_{0} \in k, y_{1} \in l\right)+\sum_{k^{\prime}, l^{\prime}=1,2, \ldots, K} \theta_{(k, l)}^{\left(k^{\prime}-k, l^{\prime}-l\right)}-\sum_{k^{\prime}, l^{\prime}=1,2, \ldots, K} \theta_{\left(k^{\prime}, l^{\prime}\right)}^{\left(k-k^{\prime}, l-l^{\prime}\right)}
\end{aligned}
$$

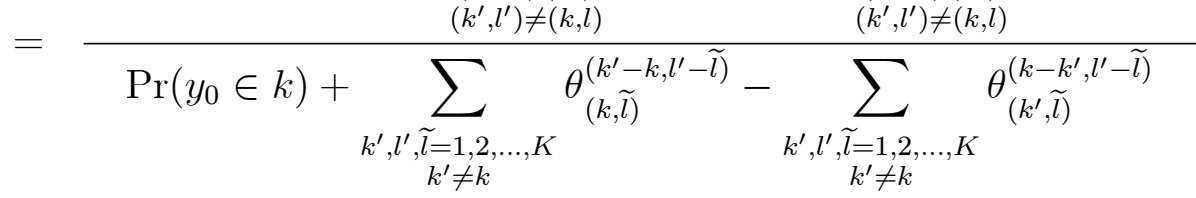

$$
\begin{aligned}
& \equiv \frac{r_{k l}+Q_{1, k l}-Q_{2, k l}}{p_{k}+Q_{3, k}-Q_{4, k}} \\
& =K\left(r_{k l}+Q_{1, k l}-Q_{2, k l}\right) \text {, }
\end{aligned}
$$

where the final line holds only in the case of equal-sized partitions. ${ }^{10} Q_{1, k l}$ measures the proportion of false negatives associated with partition $k l$ (i.e., the probability of being misclassified conditional on $k l$ being the true partition). $Q_{2, k l}$ measures the proportion of false positives associated with partition $k l$ (i.e., the probability of being misclassified conditional on $k l$ being the observed partition). Similarly, $Q_{3, k}$ and $Q_{4, k}$ measure the proportion of false negatives and positives associated with partition $k$, respectively.

The transition probabilities in (5) and (6) are not identified from the data alone. The data identify $r_{k l}$ and $p_{k}$ (and, hence, $p_{k l} \equiv r_{k l} / p_{k}$ ), but not the misclassification parameters, $\theta$. One can compute sharp bounds by searching across the unknown misclassification parameters. There are $K^{2}\left(K^{2}-1\right)$ misclassification parameters in $P_{0,1}^{*}$. However, several constraints must hold (see Appendix B). Even with these constraints, obtaining informative bounds on the transition probabilities is not possible without further restrictions. Section 3.2 considers assumptions on the $\theta$ s. Section 3.3 considers restrictions on the underlying mobility process.

Prior to continuing, it is worth relating our framework to the direct misclassification approach posited in Molinari (2008). Let $R^{*}$ denote a $K^{2} \times 1$ vector of the stacked elements of $P_{0,1}^{*}$, given by

$$
R^{*} \equiv\left[\begin{array}{llllllllll}
p_{11}^{*} & \cdots & p_{1 K}^{*} & p_{21}^{*} & \cdots & p_{2 K}^{*} & \cdots & p_{K 1}^{*} & \cdots & p_{K K}^{*}
\end{array}\right]^{\prime} .
$$

\footnotetext{
${ }^{10}$ The expression in $\sqrt{5}$ is identical to that in Gundersen and Kreider (2008, p. 368) when $K=2$.
} 
One can similarly define a $K^{2} \times 1$ vector, $R$, of observed conditional transition probabilities, given by

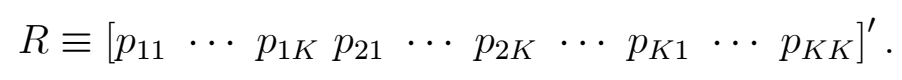

The direct misclassification approach introduces a $K^{2} \times K^{2}$ matrix of conditional misclassification probabilities, $\Pi$, such that

$$
R=\Pi R^{*}
$$

where the representative element of $\Pi, \pi_{c d}$, is given by

$$
\pi_{c d} \equiv \frac{\operatorname{Pr}\left(y_{0} \in k^{\prime}, y_{1} \in l^{\prime} \mid y_{0}^{*} \in k, y_{1}^{*} \in l\right)}{\operatorname{Pr}\left(y_{0} \in k^{\prime} \mid y_{0}^{*} \in k\right)}, \quad c, d=1, \ldots, K^{2}
$$

with $c=\left(k^{\prime}-1\right) K+l^{\prime}$ and $d=(k-1) K+l$.

This setup is identical to Molinari (2008) with the exception that the probabilities in $R^{*}$ and $R$ represent conditional transition probabilities. Molinari (2008) proceeds to derive sharp bounds given various assumptions on $\Pi$ using a nonlinear programming approach. The assumptions concerning the joint misclassification probabilities given in (4) that we consider in Section 3.2 can be written in terms of restrictions on $\Pi$. However, it is not obvious if the additional restrictions on the underlying mobility process, $R^{*}$, considered in Section 3.3 are amenable to this framework. Moreover, the estimation approach in Molianari (2008) becomes computationally challenging as the dimensionality of $R^{*}$ gets large (above 13 elements). Our code accommodates up to $5 \times 5$ transition matrices.

\subsection{Misclassification}

\subsubsection{Assumptions}

Allowing for measurement error, we obtain bounds on the elements of $P_{0,1}^{*}$, given in $(5) \cdot{ }^{11}$ We consider the following misclassification assumptions.

Assumption 1 (Classification-Preserving Measurement Error). Misreporting does not alter an observation's partition in the income distribution in either period. Formally, $\sum_{k, l} \theta_{k l}^{00}=1$

\footnotetext{
${ }^{11}$ In the interest of brevity, we focus attention from here primarily on the unconditional transition matrix. We return to the conditional transition matrix in Section 3.3.
} 
or, equivalently,

$$
\sum_{\substack{k, k^{\prime}, l, l^{\prime}=1,2, \ldots, K \\\left(k^{\prime}, l^{\prime}\right) \neq(k, l)}} \theta_{(k, l)}^{\left(k^{\prime}-k, l^{\prime}-l\right)}=0 .
$$

Assumption 2 (Maximum Misclassification Rate).

(i) (Arbitrary Misclassification) The total misclassification rate in the data is bounded from above by $Q \in(0,1)$. Formally, $1-\sum_{k, l} \theta_{k l}^{00} \leq Q$ or, equivalently,

$$
\sum_{\substack{k, k^{\prime}, l, l^{\prime}=1,2, \ldots, K \\\left(k^{\prime}, l^{\prime}\right) \neq(k, l)}} \theta_{(k, l)}^{\left(k^{\prime}-k, l^{\prime}-l\right)} \leq Q
$$

(ii) (Uniform Misclassification) The total misclassification rate in the data is bounded from above by $Q \in(0,1)$ and is uniformly distributed across partitions. Formally,

$$
\begin{aligned}
\sum_{\substack{k, k^{\prime}, l, l^{\prime}=1,2, \ldots, K \\
\left(k^{\prime}, l^{\prime}\right) \neq(k, l)}} \theta_{(k, l)}^{\left(k^{\prime}-k, l^{\prime}-l\right)} & \leq Q \\
\sum_{\substack{k^{\prime}, l, l^{\prime}=1,2, \ldots, K \\
\left(k^{\prime}, l^{\prime}\right) \neq(k, l)}} \theta_{(k, l)}^{\left(k^{\prime}-k, l^{\prime}-l\right)} & \leq \frac{Q}{K} \quad \forall k \\
\sum_{\substack{k^{\prime}, l, l^{\prime}=1,2, \ldots, K \\
\left(k^{\prime}, l^{\prime}\right) \neq(k, l)}} \theta_{(k, l)}^{\left(k^{\prime}-k, l^{\prime}-l\right)} & \leq \frac{Q}{K} \quad \forall l .
\end{aligned}
$$

Assumption 1 is quite strong, but is simply used as a benchmark. Under this assumption, measurement error is allowed as long as it does not cause observations to be classified into incorrect partitions. With equal-sized partitions, this could occur if measurement error is rank-preserving. Formally, defining $F_{t}\left(y_{i t}\right)$ and $F_{t}^{*}\left(y_{i t}^{*}\right), t=0,1$, as the marginal CDFs of observed and true income in each period, then the measurement error is rank-preserving if $F_{t}\left(y_{i t}\right)=F_{t}^{*}\left(y_{i t}^{*}\right) \forall i, t$. This is similar to Heckman et al.'s (1997) rank invariance assumption in the context of the distribution of potential outcomes in a treatment effects framework. With unequal-sized partitions, rank-preserving measurement error is not sufficient to ensure Assumption 1 holds ${ }^{12}$ Assumption 2 places restrictions on the total amount of misclassifica-

\footnotetext{
${ }^{12}$ For example, if $P_{0,1}^{*}$ is a $2 \times 2$ poverty transition matrix and all individuals over-report their income by a constant amount, then rank preservation will hold. However, some individuals may now be incorrectly classified as above the poverty line. Instead, Assumption 1 allows measurement error to be unrestricted as
} 
tion allowed in the data. As we discuss below, the amount of misclassification is dependent on the choice of $K$. As such, one could express $Q$ as $Q(K)$; we dispense with this for expositional purposes ${ }^{13}$

For the case of equal-sized partitions, misclassification is necessarily zero-sum; upward misclassification of some observations implies downward misclassification of others. Thus, even if measurement error in income is uni-directional, misclassification errors must be bidirectional. However, for the case of unequal-sized partitions, this need not be the case. In such cases, we also consider adding the following assumption.

Assumption 3 (Uni-Directional Misclassification). Misclassification occurs strictly in the upward direction. Formally,

$$
\begin{aligned}
& \theta_{(k, l)}^{\left(k^{\prime}-k, l^{\prime}-l\right)}=0 \forall k^{\prime}<k \\
& \theta_{(k, l)}^{\left(k^{\prime}-k, l^{\prime}-l\right)}=0 \forall l^{\prime}<l .
\end{aligned}
$$

Assumption 3 rules out the possibility of any false positives (negatives) occurring in the worst (best) partition. Note, this assumption is consistent with mean-reverting measurement error as long as the negative measurement errors for observations with high income are not sufficient to lead to misclassification. For example, if $P_{0,1}^{*}$ is a $2 \times 2$ poverty transition matrix, long as true poverty status is observed for all observations.

${ }^{13}$ As suggested by an anonymous reviewer, two additional restrictions might also be considered in conjuction with Assumption 2. First, one might impose independence between the misclassification probabilities in the initial and terminal periods. This implies that the misclassification probabilities

$$
\theta_{(k, l)}^{\left(k^{\prime}-k, l^{\prime}-l\right)}=\operatorname{Pr}\left(y_{0} \in k^{\prime}, y_{1} \in l^{\prime}, y_{0}^{*} \in k, y_{1}^{*} \in l\right)
$$

simplify to

$$
\theta_{(k, l)}^{\left(k^{\prime}-k, l^{\prime}-l\right)}=\alpha_{k}^{k^{\prime}-k} \bullet \beta_{l}^{l^{\prime}-l},
$$

where $\alpha_{k}^{k^{\prime}-k}\left(\beta_{l}^{l^{\prime}-l}\right)$ is the probability of being observed in partition $k^{\prime}\left(l^{\prime}\right)$ in the initial (terminal) period when the true partition is $k(l)$. This resriction reduces the number of misclassification parameters from $K^{2}\left(K^{2}-1\right)$ to $2 K(K-1)$. Second, one might wish to assume the misclassification probabilities are time invariant, impliying $\alpha_{k}^{k^{\prime}-k}=\beta_{k}^{k^{\prime}-k} \forall k$. This restriction further reduces the number of misclassification parameters to $K(K-1)$. Both assumptions are quite strong. The former restriction requires that individuals' misclassification probabilities are independent of their income history. However, one might suspect different misreporting propensities, say, for an individual who finds him/herself in poverty for the first time versus someone who has been in poverty throughout his/her lifetime. The latter restriction assumes that data accuracy and other sources of measurement error such as stigma are constant over the analysis period. In the interest of brevity, we leave the consideration of such restrictions to future work. 
Assumption 3 permits observations with true incomes exceeding the poverty threshold to underreport income, but not to a degree whereby they are misclassified as in poverty. This assumption may not hold, for instance, if some households above the poverty threshold report incomes below the poverty threshold in an attempt to qualify for means-tested transfers. Such violations seem plausible in administrative data as responses may have consequences for safety net eligibility; uni-directional errors are more likely to arise in survey data.

\subsubsection{Bounds}

Classification-Preserving Measurement Error (Assumption 1) Under Assumption 1 the sampling process identifies the transition probabilities despite the presence of measurement error, yielding the following proposition.

Proposition 1. Under Assumption 1 the transition probabilities are nonparametrically identified by

$$
\begin{aligned}
p_{k l}^{*} & =\frac{\operatorname{Pr}\left(y_{0} \in k, y_{1} \in l\right)}{\operatorname{Pr}\left(y_{0} \in k\right)} \\
& =\frac{\mathrm{E}\left[I\left(y_{0} \in k, y_{1} \in l\right)\right]}{\mathrm{E}\left[I\left(y_{0} \in k\right)\right]},
\end{aligned}
$$

where $\mathrm{E}[\cdot]$ is the expectation operator and $I(\cdot)$ is the indicator function. Proof: See Appendix C.

Estimation proceeds by replacing the terms with their sample analogs, given by

$$
\begin{aligned}
\widehat{p}_{k l} & =\frac{\sum_{i} \mathrm{I}\left(y_{0 i} \in k, y_{1 i} \in l\right)}{\sum_{i} \mathrm{I}\left(y_{0 i} \in k\right)} \\
& =\frac{K}{N} \sum_{i} \mathrm{I}\left(y_{0 i} \in k, y_{1 i} \in l\right),
\end{aligned}
$$

where the last line follows in the case of equal-sized partitions.

Maximum Misclassification Rate (Assumption 2) Under Assumption 2 with $Q>0$, the transition probabilities are no longer nonparametrically identified. We have the following propositions. 
Proposition 2. Consider a transition matrix, $P_{0,1}^{*}$, with equal-sized partitions. The transition probabilities are bounded sharply by

$$
\begin{aligned}
p_{k l}^{*} \in & {\left[\max \left\{K\left(r_{k l}-\widetilde{Q}\right), 1-\sum_{\substack{l^{\prime}=1,2, \ldots, K \\
l^{\prime} \neq l}} U B_{k l^{\prime}}, 1-\sum_{\substack{k^{\prime}=1,2, \ldots, K \\
k^{\prime} \neq k}} U B_{k^{\prime}}, 0\right\},\right.} \\
& \min \left\{K\left(r_{k l}+\widetilde{Q}\right), 1-\sum_{\substack{l^{\prime}=1,2, \ldots, K \\
l^{\prime} \neq l}} L B_{k l^{\prime}}, 1-\sum_{\substack{k^{\prime}=1,2, \ldots, K \\
k^{\prime} \neq k}} L B_{k^{\prime} l}, 1\right\},
\end{aligned}
$$

where $L B_{k l} \equiv \max \left\{K\left(r_{k l}-\widetilde{Q}\right), 0\right\}, U B_{k l} \equiv \min \left\{K\left(r_{k l}+\widetilde{Q}\right), 1\right\}$, and $\widetilde{Q}=Q / 2$ under Assumption 2(i) and $\widetilde{Q}=Q / K$ under Assumption 2(ii). Proof: See Appendix C.

Proposition 3. Consider a transition matrix, $P_{0,1}^{*}$, with unequal-sized partitions. Under Assumption 2(i), the transition probabilities are bounded sharply by

$$
p_{k l}^{*} \in\left[\max \left\{\frac{r_{k l}-Q}{p_{k}}, 1-\sum_{\substack{l^{\prime}=1,2, \ldots, K \\ l^{\prime} \neq l}} U B_{k l^{\prime}}, 0\right\}, \min \left\{\frac{r_{k l}+Q}{p_{k}}, 1-\sum_{\substack{l^{\prime}=1,2, \ldots, K \\ l^{\prime} \neq l}} L B_{k l^{\prime}}, 1\right\}\right],
$$

where $L B_{k l} \equiv\left(r_{k l}-Q\right) / p_{k}$ and $U B_{k l} \equiv\left(r_{k l}+Q\right) / p_{k}$. Under Assumption 2(ii), the transition probabilities are bounded sharply by

$$
\begin{aligned}
p_{k l}^{*} \in & {\left[\max \left\{\frac{r_{k l}-Q / K}{p_{k}}, 1-\sum_{\substack{l^{\prime}=1,2, \ldots, K \\
l^{\prime} \neq l}} U B_{k l^{\prime}}, 0\right\},\right.} \\
& \left.\min \left\{\frac{r_{k l}+Q / K}{p_{k}-\min \left\{Q / K, p_{k}\right\}}, 1-\sum_{\substack{l^{\prime}=1,2, \ldots, K \\
l^{\prime} \neq l}} L B_{k l^{\prime}}, 1\right\}\right],
\end{aligned}
$$

where $L B_{k l} \equiv \max \left\{\left(r_{k l}-Q / K\right) / p_{k}\right\}$ and $U B_{k l} \equiv \min \left\{\left(r_{k l}+Q / K\right) /\left(p_{k}-\min \left\{Q / K, p_{k}\right\}\right), 1\right\}$. Proof: See Appendix C.

Estimation of the bounds in Propositions 2 and 3 proceeds by replacing $r_{k l}$ and $p_{k}$ with their sample analogs and then verifying that the required conditions are met.

Uni-Directional Misclassification (Assumption 3) For simplicity, we only consider Assumption 3 in the case of a $2 \times 2$ transition matrix. We have the following proposition. 
Proposition 4. Under Assumption 3, the four elements of a $2 \times 2$ transition matrix with unequal-sized partitions are bounded sharply by

$$
\begin{aligned}
& p_{11}^{*} \in\left[\max \left\{\frac{r_{11}}{p_{1}+\min \left\{\widetilde{Q}, 1-p_{1}\right\}}, 1-U B_{12}, 0\right\}, \min \left\{\frac{r_{11}+\widetilde{Q}}{p_{1}}, 1-L B_{12}, 1\right\}\right] \\
& p_{12}^{*} \in\left[\max \left\{\frac{r_{12}-\widetilde{Q}}{p_{1}}, 1-U B_{11}, 0\right\}, \min \left\{\frac{r_{12}+\min \left\{\widetilde{Q}, 1-p_{1}\right\}}{p_{1}+\min \left\{\widetilde{Q}, 1-p_{1}\right\}}, 1-L B_{11}, 1\right\}\right] \\
& p_{21}^{*} \in\left[\max \left\{\frac{r_{21}-\min \left\{\widetilde{Q}, p_{2}\right\}}{p_{2}-\min \left\{\widetilde{Q}, p_{2}\right\}}, 1-U B_{22}, 0\right\}, \min \left\{\frac{r_{21}+\widetilde{Q}}{p_{2}-\widetilde{Q}}, 1-L B_{22}, 1\right\}\right] \\
& p_{22}^{*} \in\left[\max \left\{\frac{r_{22}-\widetilde{Q}}{p_{2}}, 1-U B_{21}, 0\right\}, \min \left\{\frac{r_{22}}{p_{2}-\min \left\{\widetilde{Q}, p_{2}\right\}}, 1-L B_{21}, 1\right\}\right],
\end{aligned}
$$

where $L B_{k l}$ and $U B_{k l}$ denote the lower and upper bounds of $p_{k l}^{*}$, respectively. Under Assumption 2(i), $\widetilde{Q}=Q$ and $\widetilde{\widetilde{Q}}=0$. Under Assumption 2(ii), $\widetilde{Q}=Q / 2$ and $\widetilde{\widetilde{Q}}=\min \left\{\widetilde{Q}, p_{2}\right\}$. Proof: See Appendix $C$.

Estimation of the bounds are straightforward using the appropriate sample analogs and then verifying that the required conditions are met.

\subsection{Restrictions}

Propositions 244 provide bounds on transition probabilities considering only restrictions on the misclassification process. Here, we explore the identifying power of incorporating restrictions on the mobility process. The restrictions may be imposed alone or in combination.

\subsubsection{Shape Restrictions}

Shape restrictions place inequality constraints on the population transition probabilities ${ }^{14}$ Here, we consider imposing shape restrictions assuming that large transitions are less likely than smaller ones.

Assumption 4 (Shape Restrictions). The transition probabilities are weakly decreasing in the size of the transition. Formally, $p_{k l}^{*}$ is weakly decreasing in $|k-l|$, the absolute difference between $k$ and $l$.

\footnotetext{
${ }^{14}$ See Chetverikov et al. (2018) for a recent review of the use of shape restrictions in economics.
} 
This assumption implies that within each row or each column of the transition matrix, the diagonal element (i.e., the conditional staying probability) is the largest. The remaining elements decline weakly monotonically moving away from the diagonal element. This assumption, which may be plausible if large jumps in income are less common than small ones, leads to the following proposition.

Proposition 5. Denote the bounds on $p_{k l}^{*}$ under some combination of Assumptions 2 and 3 as

$$
p_{k l}^{*} \in\left[L B_{k l}, U B_{k l}\right] .
$$

Adding Assumption 4 implies the following sharp bounds:

$$
\begin{aligned}
& p_{k l}^{*} \in\left[\max \left\{\sup _{l^{\prime}=1, \ldots, K} L B_{k l^{\prime}}, \sup _{k^{\prime}=1, \ldots, K} L B_{k^{\prime} l}\right\}, U B_{k l}\right] \text { if } k=l \\
& p_{k l}^{*} \in\left[\max \left\{\sup _{l^{\prime} \geq l} L B_{k l^{\prime}}, \sup _{k^{\prime} \leq k} L B_{k^{\prime} l}\right\}, \min \left\{\inf _{k \leq l^{\prime} \leq l} U B_{k l^{\prime}}, \inf _{k \leq k^{\prime} \leq l} U B_{k^{\prime} l}\right\}\right] \text { if } k<l \\
& p_{k l}^{*} \in\left[\max \left\{\sup _{l^{\prime} \leq l} L B_{k l^{\prime}}, \sup _{k^{\prime} \geq k} L B_{k^{\prime} l}\right\}, \min \left\{\inf _{l \leq l^{\prime} \leq k} U B_{k l^{\prime}}, \inf _{l \leq k^{\prime} \leq k} U B_{k^{\prime} l}\right\}\right] \text { if } k>l .
\end{aligned}
$$

Proof: See Appendix C.

Estimation is straightforward given estimates of the preliminary bounds, $L B_{k l}$ and $U B_{k l}$.

\subsubsection{Level Set Restrictions}

Level set restrictions place equality constraints on population transition probabilities across observations with different observed attributes (Manski 1990; Lechner 1999).

Assumption 5 (Level Set Restrictions). The conditional transition probabilities, given in (3), are constant across a range of conditioning values. Formally, $p_{k l}^{*}(x)$ is constant for all $x \in \mathcal{A}_{x} \subset \mathcal{R}_{m}$, where $x$ is an m-dimensional vector.

For instance, if $x$ denotes the age of an individual in years, one might wish to assume that $p_{k l}^{*}(z)$ is constant for all $z$ within a fixed window around $x$. 
From (3) and (5), we have

$$
\begin{aligned}
& \operatorname{Pr}\left(y_{0} \in k, y_{1} \in l \mid X=x\right)+\sum_{\substack{k^{\prime}, l^{\prime}=1,2, \ldots, K \\
\left(k^{\prime}, l^{\prime}\right) \neq(h, l)}} \theta_{(k, l)}^{\left(k^{\prime}-k, l^{\prime}-l\right)}(x)-\sum_{\substack{k^{\prime}, l^{\prime}=1,2, \ldots, K \\
\left(k^{\prime}, l^{\prime}\right) \neq(l, l)}} \theta_{\left(k^{\prime}, l^{\prime}\right)}^{\left(k-k^{\prime}, l-l^{\prime}\right)}(x)
\end{aligned}
$$

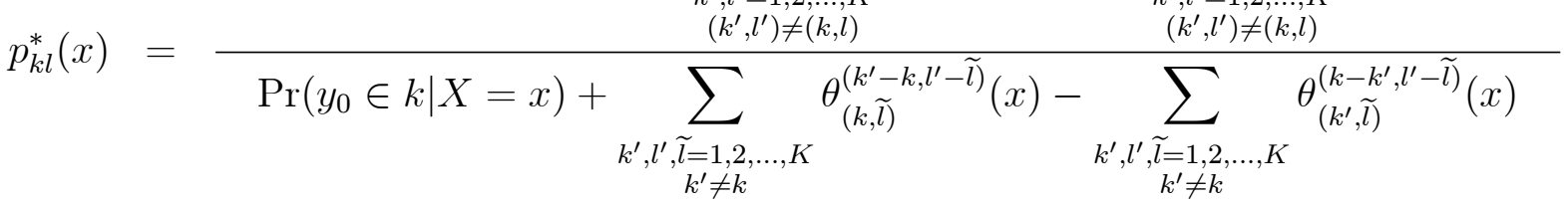

$$
\begin{aligned}
& \equiv \frac{r_{k l}(x)+Q_{1, k l}(x)-Q_{2, k l}(x)}{p_{k}(x)+Q_{3, k}(x)-Q_{4, k}(x)}
\end{aligned}
$$

where now $Q_{j, .}(x), j=1, \ldots, 4$, represent the proportions of false positives and negatives conditional on $x$. As such, we also consider the following assumption regarding the conditional misclassification probabilities.

Assumption 6 (Independence). Misclassification rates are independent of the observed attributes of observations, $x$. Formally,

$$
\theta_{(k, l)}^{\left(k^{\prime}-k, l^{\prime}-l\right)}(x)=\theta_{(k, l)}^{\left(k^{\prime}-k, l^{\prime}-l\right)}, \forall k, k^{\prime}, l, l^{\prime}, x
$$

The plausibility of Assumption 6 depends on one's conjectures concerning the measurement error process. However, two points are important to bear in mind. First, the misclassification probabilities, $\theta_{(k, l)}^{\left(k^{\prime}-k, l^{\prime}-l\right)}$, are specific to a pair of true and observed partitions. As a result, even if misclassification is more likely at certain parts of the income distribution and $x$ is correlated with income, this does not necessarily invalidate Assumption 6. Second, Assumption 6 does not imply that misclassification rates are independent of all individual attributes, only those included in the variables used to define the level set restrictions. This leads to the following proposition.

Proposition 6. Denote the bounds for $p_{k l}^{*}(x)$ under some combination of Assumptions $2 / 4$ and 6 as

$$
p_{k l}^{*}(x) \in[L B(x), U B(x)] .
$$

Adding Assumption 5 implies the following sharp bounds on the conditional transition probabilities:

$$
p_{k l}^{*}(x) \in\left[\sup _{z \in \mathcal{A}_{x}} L B(z), \inf _{z \in \mathcal{A}_{x}} U B(z)\right] .
$$

Assuming $X$ is discrete, sharp bounds on the unconditional transition probabilities are given 
as

$$
p_{k l}^{*} \in\left[\sum_{x} \operatorname{Pr}(X=x)\left(\sup _{z \in \mathcal{A}_{x}} L B(z)\right), \sum_{x} \operatorname{Pr}(X=x)\left(\inf _{z \in \mathcal{A}_{x}} U B(z)\right)\right] .
$$

Proof: See Manski and Pepper (2000).

To operationalize Proposition 6, bounds on the conditional transition probabilities in (8) must be obtained. This is done in the following corollaries.

Corollary 6.1. Consider a transition matrix, $P_{0,1}^{*}$, with equal- or unequal-sized partitions. Under Assumption 2(i), $p_{k l}^{*}(x)$ is bounded sharply by

$p_{k l}^{*}(x) \in\left[\max \left\{\frac{r_{k l}(x)-\ddot{Q}}{p_{k}(x)}, 1-\sum_{\substack{l^{\prime}=1,2, \ldots, K \\ l^{\prime} \neq l}} U B_{k l^{\prime}}(x), 0\right\}, \min \left\{\frac{r_{k l}(x)+\ddot{Q}}{p_{k}(x)}, 1-\sum_{\substack{l^{\prime}=1,2, \ldots, K \\ l^{\prime} \neq l}} L B_{k l^{\prime}}(x), 1\right\}\right]$

where $L B_{k l}(x) \equiv \max \left\{\left(r_{k l}(x)-\ddot{Q}\right) / p_{k}(x)\right\}, U B_{k l} \equiv \min \left\{\left(r_{k l}(x)+\ddot{Q}\right) / p_{k}(x), 1\right\}$,

$$
\ddot{Q}=\left\{\begin{array}{cl}
\widetilde{Q} & \text { under Assumption } 6 \\
\widetilde{Q} / \operatorname{Pr}(X=x) & \text { otherwise }
\end{array}\right.
$$

and

$$
\widetilde{Q}=\left\{\begin{array}{cl}
Q / 2 & \text { for equal-sized partitions } \\
Q & \text { for unequal-sized partitions }
\end{array}\right.
$$

Proof: See Appendix C.

Corollary 6.2. Consider a transition matrix, $P_{0,1}^{*}$, with equal- or unequal-sized partitions. Under Assumption 2(ii), $p_{k l}^{*}(x)$ is bounded sharply by

$$
\begin{aligned}
p_{k l}^{*}(x) \in & {\left[\max \left\{\frac{r_{k l}(x)-\widetilde{Q}}{p_{k}(x)}, 1-\sum_{\substack{l^{\prime}=1,2, \ldots, K \\
l^{\prime} \neq l}} U B_{k l^{\prime}}(x), 0\right\},\right.} \\
& \left.\min \left\{\frac{r_{k l}(x)+\widetilde{Q}}{p_{k}(x)-\min \left\{\widetilde{Q}, p_{k}(x)\right\}}, 1-\sum_{\substack{l^{\prime}=1,2, \ldots, K \\
l^{\prime} \neq l}} L B_{k l^{\prime}}(x), 1\right\}\right]
\end{aligned}
$$

where $L B_{k l}(x) \equiv \max \left\{\left(r_{k l}(x)-\widetilde{Q}\right) / p_{k}(x)\right\}, U B_{k l} \equiv \min \left\{\left(r_{k l}(x)+\widetilde{Q}\right) /\left(p_{k}(x)-\min \left\{\widetilde{Q}, p_{k}(x)\right\}\right), 1\right\}$, and

$$
\widetilde{Q}=\left\{\begin{array}{cl}
Q / K & \text { under Assumption } 6 \\
Q / K \operatorname{Pr}(X=x) & \text { otherwise }
\end{array}\right.
$$

Proof: See Appendix C. 
Corollary 6.3. Consider a $2 \times 2$ transition matrix, $P_{0,1}^{*}$, with unequal-sized partitions. Under Assumption 3, the four elements are bounded sharply by

$$
\begin{aligned}
& p_{11}^{*}(x) \in \max \left\{\frac{r_{11}(x)}{\min \left\{p_{1}(x)+\widetilde{Q}, 1\right\}}, 1-U B_{12}(x), 0\right\}, \min \left\{\frac{r_{11}(x)+\widetilde{Q}}{p_{1}(x)}, 1-L B_{12}(x), 1\right\} \\
& p_{12}^{*}(x) \in \max \left\{\frac{r_{12}(x)-\widetilde{Q}}{p_{1}(x)}, 1-U B_{11}(x), 0\right\}, \min \left\{\frac{r_{12}(x)+\min \left\{\widetilde{Q}, 1-p_{1}(x)\right\}}{p_{1}(x)+\min \left\{\widetilde{Q}, 1-p_{1}(x)\right\}}, 1-L B_{11}(x), 1\right\} \\
& p_{21}^{*}(x) \in \max \left\{\frac{r_{21}(x)-\min \left\{\widetilde{Q}, p_{2}(x)\right\}}{p_{2}(x)-\min \left\{\widetilde{Q}, p_{2}(x)\right\}}, 1-U B_{22}(x), 0\right\}, \min \left\{\frac{r_{21}(x)+\widetilde{Q}}{p_{2}(x)-\widetilde{\widetilde{Q}}}, 1-L B_{22}(x), 1\right\} \\
& p_{22}^{*}(x) \in \max \left\{\frac{r_{22}(x)-\widetilde{Q}}{p_{2}(x)}, 1-U B_{21}(x), 0\right\}, \min \left\{\frac{r_{22}(x)}{p_{2}(x)-\min \left\{\widetilde{Q}, p_{2}(x)\right\}}, 1-L B_{21}(x), 1\right\}
\end{aligned}
$$

where $L B_{k l}(x)$ and $U B_{k l}(x)$ denote the lower and upper bounds of $p_{k l}^{*}(x)$, respectively,

$$
\widetilde{\widetilde{Q}}=\left\{\begin{array}{cl}
0 & \text { under Assumption } 2(i) \\
\min \left\{\widetilde{Q}, p_{2}(x)\right\} & \text { under Assumption } 2(i i)
\end{array}\right.
$$

and

$$
\widetilde{Q}=\left\{\begin{array}{cl}
Q & \text { under Assumptions } 2(i) \text { and } 6 \\
Q / \operatorname{Pr}(X=x) & \text { under Assumption } 2(i) \\
Q / 2 & \text { under Assumptions } 2(i i) \text { and } 6 \\
Q / 2 \operatorname{Pr}(X=x) & \text { under Assumption } 2(i i)
\end{array}\right.
$$

Proof: See Appendix $C$.

Under Corollaries 6.1, 6.2, and 6.3, estimation of the bounds for $p_{k l}^{*}(x)$ are straightforward using the appropriate sample analogs and minimizing (maximizing) the lower (upper) bound subject to the appropriate constraints. Upon obtaining bounds for $p_{k l}^{*}(x)$, sharp bounds for the conditional and unconditional transition probabilities are given in (9) and (10) 15

Before continuing, it is worth pointing out a special case of level set restrictions when the conditioning variable, $x$, represents time. For example, one might separately bound transition matrices from $t=0 \rightarrow 1$ and $t=1 \rightarrow 2$ and then impose the restriction that mobility is constant across the two time periods. Here, the level set restriction is identical to a stationarity assumption about the Markov process governing the outcome variable. This is formalized in the following assumption and proposition.

\footnotetext{
${ }^{15}$ Note, there is no assurance that the bounds under Assumption 5 , but without Assumption 6, will be narrower than the corresponding bounds without Assumption 5 .
} 
Assumption 7 (Stationarity). The transition matrix is constant across two consecutive periods. Formally,

$$
P_{t, t+1}^{*}=P_{t+1, t+2}^{*}
$$

Proposition 7. Let $p_{k l}^{*}(t, t+1)$ represent the elements of $P_{t, t+1}^{*}$. Denote the bounds for $p_{k l}^{*}(t, t+1)$ under some combination of Assumptions 20 6 as

$$
p_{k l}^{*}(t, t+1) \in[L B(t, t+1), U B(t, t+1)] .
$$

Define the elements and corresponding bounds similarly for $P_{t+1, t+2}^{*}$. Adding Assumption 7 implies the following sharp bounds on the elements of $P^{*}=P_{t, t+1}^{*}=P_{t+1, t+2}^{*}$

$$
p_{k l}^{*} \in[\max \{L B(t, t+1), L B(t+1, t+2)\}, \min \{U B(t, t+1), U B(t+1, t+2)\}],
$$

where $p_{k l}^{*}$ refers to the elements of $P^{*}$. Proof: Follows directly from Proposition 6 ,

\subsubsection{Monotonicity Assumptions}

Monotonicity restrictions place inequality constraints on population transition probabilities across observations with different observed attributes (Manski and Pepper 2000; Chetverikov et al. 2018).

Assumption 8 (Monotonicity). The conditional probability of upward mobility is weakly increasing in a vector of attributes, $u$, and the conditional probability of downward mobility is weakly decreasing in the same vector of attributes. Formally, if $u_{2} \geq u_{1}$, then

$$
\begin{aligned}
p_{11}^{*}\left(u_{1}\right) & \geq p_{11}^{*}\left(u_{2}\right) \\
p_{K K}^{*}\left(u_{1}\right) & \leq p_{K K}^{*}\left(u_{2}\right) \\
p_{k l}^{*}\left(u_{1}\right) & \leq p_{k l}^{*}\left(u_{2}\right) \quad \forall l>k \\
p_{k l}^{*}\left(u_{1}\right) & \geq p_{k l}^{*}\left(u_{2}\right) \quad \forall l<k .
\end{aligned}
$$

For instance, if $u$ denotes the education of an individual, one might wish to assume that the probability of upward (downward) mobility is no lower (higher) for individuals with more education. Note, the monotonicity assumption provides no information on the conditional staying probabilities, $p_{k k}^{*}(u)$, for $k=2, \ldots, K-1$.

This leads to the following proposition. 
Proposition 8. Denote the bounds for $p_{k l}^{*}(u)$ under some combination of Assumptions $2[6$ as

$$
p_{k l}^{*}(u) \in[L B(u), U B(u)] .
$$

Adding Assumption 8 implies the following sharp bounds on the conditional transition probabilities

$$
\begin{aligned}
p_{11}^{*}(u) & \in\left[\sup _{u \leq u_{1}} L B\left(u_{1}\right), \inf _{u_{2} \leq u} U B\left(u_{2}\right)\right] \\
p_{K K}^{*}(u) & \in\left[\sup _{u_{1} \leq u} L B\left(u_{1}\right), \inf _{u \leq u_{2}} U B\left(u_{2}\right)\right] \\
p_{k l}^{*}(u) & \in\left[\sup _{u_{1} \leq u} L B\left(u_{1}\right), \inf _{u \leq u_{2}} U B\left(u_{2}\right)\right] \quad \forall l>k \\
p_{k l}^{*}(u) & \in\left[\sup _{u \leq u_{1}} L B\left(u_{1}\right), \inf _{u_{2} \leq u} U B\left(u_{2}\right)\right] \quad \forall l<k
\end{aligned}
$$

Assuming $U$ is discrete, sharp bounds on the unconditional transition probabilities are given as

$$
p_{k l}^{*} \in\left[\sum_{u} \operatorname{Pr}(U=u)\left(\sup _{u_{1} \leq u} L B\left(u_{1}\right)\right), \sum_{u} \operatorname{Pr}(U=u)\left(\inf _{u_{1} \geq u} U B\left(u_{1}\right)\right)\right] .
$$

Proof: This is a simple extension of Manski and Pepper (2000, Proposition 1 and Corollary 1).

\subsection{Summary Mobility Measures}

Several scalar measures of mobility considered in the literature are derived directly from the elements of the transition matrices. The Prais (1955) measure of mobility captures the expected exit time from partition $k$ and is given by

$$
\frac{1}{1-p_{k k}^{*}}, k=1, \ldots, K
$$

Bradbury (2016) defines measures of upward and downward mobility that account for the size of the partitions. The upward mobility measure is given by

$$
U M=\frac{K}{K-1}\left(1-p_{11}^{*}\right)
$$


downward mobility is given by

$$
D M=\frac{K}{K-1}\left(1-p_{K K}^{*}\right)
$$

Mobility is decreasing in the value of the Prais measure; increasing in the remaining two measures. The measures in (11)-(13) can be sharply bounded in a straightforward manner using sharp bounds on the individual conditional staying probabilities since each measure depends on only one element from the transition matrix.

\subsection{Properties}

\subsubsection{Bias Correction}

In most of the cases considered here, estimates of the bounds are obtained via plug-in estimators relying on infima and suprema. Such estimators are biased in finite samples, producing bounds that are too narrow (Kreider and Pepper 2008). To circumvent this issue, a bootstrap bias correction is typically used in the literature on partial identification. Denote the plug-in estimators of the lower and upper bounds under some set of the preceding assumptions as $\widehat{L B}$ and $\widehat{U B}$, respectively. The bootstrap bias corrected estimates are given by

$$
\begin{aligned}
& \widehat{L B}_{c}=2 \widehat{L B}-\mathrm{E}^{*}[\widehat{L B}] \\
& \widehat{U B}_{c}=2 \widehat{U B}-\mathrm{E}^{*}[\widehat{U B}]
\end{aligned}
$$

where $\widehat{L B}_{c}$ and $\widehat{U B}_{c}$ denote the bootstrap bias corrected estimates and $\mathrm{E}^{*}[\cdot]$ denotes the expectation operator with respect to the bootstrap distribution. See Kreider and Pepper (2008) and the references therein. However, there is an added complication here. Because

\footnotetext{
${ }^{16} \mathrm{~A}$ fourth measure derived from the transition matrix is the Immobility Ratio, attributable to Shorrocks (1978). The measure is given by

$$
I R=\frac{K-\operatorname{tr}\left(P_{0,1}^{*}\right)}{K-1},
$$

where $\operatorname{tr}(\cdot)$ denotes the trace of a matrix. Since the trace is a function of multiple elements of the matrix one from each row and column - bounds on $I R$ using the upper and lower bounds on the diagonal elements of the trace under Assumption 2(i)] are not sharp. They are sharp under Assumption 2(ii)] Future work may wish to consider sharp bounds on $I R$ under arbitrary errors.
} 
we are estimating bounds on probabilities, the upper (lower) bound is constrained by one (zero). It is well known that the traditional bootstrap does not work for parameters at or near the boundary of the parameter space (Andrews 2000). Instead, we employ subsampling, using replicate samples with N/2 observations (Andrews and Guggenberger 2009; MartínezMuñoz and Suáreza 2010) ${ }^{17}$

\subsubsection{Inference}

A substantial body of literature exists on inference in partial identification models. Early work focused on confidence regions for the identified set (Stoye 2009). Imbens and Manski (2004) instead derive confidence regions for the partially identified parameter of interest. Here, inference is handled via subsampling and the Imbens-Manski (2004) correction to obtain $90 \%$ confidence intervals (CIs) ${ }^{18}$ As with the bias correction, we set the size of the replicate samples to $N / 2$.

Some comments on this choice is necessary as there has been much recent work on inference in partially identified models; Bontemps and Magnac (2017), Canay and Shaikh (2017), and Ho and Rosen (2017) provide excellent reviews. For instance, intersection bounds, (conditional) moment inequality, and random set theory and Bayesian approaches are also used for estimation and inference in partial identification models. When a single parameter is being bounded, the endpoints of the bounds are asymptotically normal, and the sample is randomly drawn from an infinite population, then the approach in Imbens and Manski (2004) or Stoye (2009) is applicable and straightforward. However, when the endpoints are obtained via intersection bounds, as in the case of level set or monotonicity restrictions, then methods such as those provided in Chernozhukov et al. (2007) or Chernozhukov et al.

\footnotetext{
${ }^{17}$ We employ sub-sampling (without replacement) rather than an $m$-bootstrap (with replacement), where $m<N$, as sub-sampling is valid under weaker assumptions (Horowitz 2001). Noneless, our Stata code allows for both options. Moreover, we set $m=N / 2$ as it is unlikely that an optimal, data-driven choice of $m$ is available (or computationally feasible in the present context). Politis et al. (1999, p. 61) state that "subsampling has some asymptotic validity across a broad range of choices for the subsample size" as long as $m / N \rightarrow 0$ and $m \rightarrow \infty$ as $N \rightarrow \infty$. Martínez-Muñoz and Suáreza (2010, p. 143) note that setting $m=N / 2$ is "typical."

${ }^{18}$ Since a $K \times K$ transition matrix entails the estimation of $K(K-1)$ free parameters, one might be concerned with issues related to multiple hypothesis testing depending on the nature of the hypotheses being considered. While not considered here, our code does allow for a Bonferonni correction if one so chooses.
} 
(2013) are available depending on whether the conditioning variable is discrete or continuous. However, we do not pursue such approaches here for two reasons. First, it is not clear how to convert all the restrictions we wish to consider into a set of (conditional) moments. Second, in the case of our level set or monotonicity restrictions, the method in Chernozhukov et al. (2013) seems applicable if one is interested in bounds and confidence regions for the conditional transition probabilities, $p_{k l}^{*}(x)$ and $p_{k l}^{*}(u)$. However, as we are ultimately interested in bounds for the unconditional transition probabilities, $p_{k l}^{*}$, which are weighted averages of the bounds on the conditional transition probabilities, application of this method is not obvious.

\section{U.S. Mobility}

\subsection{Data}

To assess US intragenerational mobility, we use panel data from the Survey of Income and Program Participation (SIPP). Collected by the US Census Bureau, SIPP is a rotating, nationally representative longitudinal survey of households. Begun in 1984, SIPP collects detailed income data as well as data on a host of other economic and demographic attributes. Households in the SIPP are surveyed over a multi-year period ranging from two and a half years to four years. Then, a new sample of households are drawn. The sample sizes range from approximately 14,000 to 52,000 households. Here, we use the 2004 and 2008 panels to examine mobility leading up to the Great Recession and during the early recovery period. For the 2004 panel, the initial period is November 2003 and the terminal period is October 2007. For the 2008 panel, the initial period is June 2008 and the terminal period is September 2012. Thus, we investigate household-level income dynamics over two separate four-year windows. We also assess mobility pooling the two panels.

For the analysis, the outcome variable is derived from total monthly household income (variable THTOTINC). This includes income from all household members and sources: labor market earnings, pensions, social security income, interest dividends, and other income sources. When analyzing the $2 \times 2$ poverty matrix, we determine poverty status for each household in each period by comparing income with the SIPP-reported poverty threshold 
for the household (variable RHPOV). When analyzing general mobility, we estimate $3 \times 3$ matrices based on terciles of the income distribution in each period. However, to adjust for household composition, we construct three different measures of so-called equivalized household income ${ }^{19}$ Adjusting income for household size when drawing welfare or policy conclusions is known to be crucial (e.g., Chiappori 2016). In our baseline analysis, we use OECD equivalized household income (OECD 1982) ${ }^{20}$ As alternatives, we also construct OECD-modified equivalized household income (Haagenars et al. 1994) and per capita household income ${ }^{21}$ Specifically, the OECD (OECD-modified) equivalence scale assigns a value of one to the first household member, 0.7 (0.5) to each additional adult, and of $0.5(0.3)$ to each child. In contrast, the per capita measure assigns a value of one to all household members. In the interest of brevity, results based on these alternative equivalence scales are relegated to Appendix E.

In constructing our estimation sample, we use only the initial and terminal wave for each panel. The sample, by necessity, must be balanced. Households with any invalid or missing information on the relevant variables are excluded. Finally, we restrict the sample to households where the head is between 25 and 65 years old in the initial period. The sample size for the 2004 panel is 7,834 and for the 2008 panel is 16,006 Summary statistics are presented in Table 1.

When assessing the two panels separately and imposing level set restrictions, we use age of the household head in the initial period. Specifically, we group households into tenyear age bins $(25-34, \ldots, 55-65)$ and impose the restriction that mobility is constant across adjacent bins. For example, we tighten the bounds on mobility for households where the

\footnotetext{
${ }^{19}$ There is no need to adjust income for household size when estimating the poverty transition matrix since the poverty threshold already accounts for differences in household composition.

${ }^{20}$ OECD equivalized household income for an individual household is defined as $Y / N$, where $Y$ is total household income, $N=1+0.7(A-1)+0.5 C$, and $A(C)$ is the total number of adults (children) in the household.

${ }^{21}$ OECD-modified equivalized household income for an individual household is defined as $Y / N$, where $Y$ is total household income, $N=1+0.5(A-1)+0.3 C$, and $A(C)$ is the total number of adults (children) in the household.

${ }^{22}$ The 2004 panel contains 10,503 households observed in the initial and terminal periods. Two observations are dropped due to negative household income. The remainder are dropped because the household head is outside the 25-65 year old age range. The 2008 panel panel contains 21,616 households observed in the initial and terminal periods. 88 observations are dropped due to negative or missing household income. The remainder are dropped because the household head is outside the 25-65 year old age range.
} 
head is, say, 35-44 by assuming that mobility is constant across households where the head is 25-34, 35-44, and 45-54. When pooling the two panels and imposing level set restrictions, we combine the age of household head restriction used in the case of separate panels with a stationarity assumption that mobility is constant across the two panels. For example, we tighten the bounds on mobility for households where the head is, say, 35-44 in the initial period of the 2004 panel by assuming that mobility is constant across households where the head is 25-34, 35-44, and 45-54 in the 2004 and 2008 panels.

When imposing the monotonicity restrictions, we use the education of the household head in the initial period. Here, households are grouped into three bins (high school graduate and below, some college but less than a four-year degree, and at least a four-year college degree).

\subsection{Results}

\subsubsection{Poverty Transition Matrix}

Results for the $2 \times 2$ poverty transition matrix are presented in Tables $2-4$ Overall, the observed poverty rate declined from $11.8 \%$ to $10.7 \%$ in the first panel (November 2003 to October 2007) and held constant at 12.6\% in the second panel (June 2008 to September 2012); see Table 1. Turning to mobility, under the baseline assumption of ClassificationPreserving Measurement Error (Table 2, Panel I) the probability of a household remaining in poverty across the initial and terminal periods in the first (second) SIPP panel is 0.448 (0.462), while the probability of remaining out of poverty is $0.939(0.923){ }^{24}$ Thus, observed transitions out of (into) poverty are higher in the first (second) SIPP panel (transition out of poverty: 0.552 versus 0.538 ; transitions into poverty: 0.061 versus 0.077 ). This is not surprising since the second SIPP panel spans the end of the Great Recession and the early part of the recovery.

\footnotetext{
${ }^{23}$ In all cases, we use 25 replicate samples for the subsampling bias correction and 100 replicate samples to construct 90\% Imbens-Manski (2004) confidence intervals via subsampling using $m=N / 2$ without replacement. For brevity, we do not report bounds based on all possible combinations of restrictions. Unreported results are available upon request.

${ }^{24}$ Throughout the analysis, poverty status is measured only at the initial and terminal period. Thus, for example, "remaining in poverty" does not mean a household is necessarily in poverty continuously over the four-year period. For expositional purposes, however, we describe the results in terms of remaining in or out of poverty.
} 
Misclassification Assumptions Panels II and III in Table 2 allow for misclassification, but impose arbitrary (Assumption 2(i)] and uniform (Assumption 2(ii) errors, respectively. The assumed maximum misclassification rate is $10 \%(Q=0.10)$. The rationale for this choice is discussed in Appendix D; we also explore sensitivity to this choice below. In Panel II the bounds are nearly uninformative on the mobility of households in poverty in the initial period in both SIPP panels. Thus, a relatively small amount of arbitrary misclassification results, in the absence of other information, in an inability to say anything about the four-year mobility rates of households initially in poverty. This arises because the maximum allowable misclassification rate is nearly as large as the fraction of the sample reported to be in poverty in the initial period. For households initially above the poverty line, more can be learned even in the presence of an arbitrary $10 \%$ misclassification rate as this includes the majority of the sample. First, the probability of remaining out of poverty four years later is at least 0.825 (0.808) in the first (second) SIPP panel. ${ }^{25}$ Second, the probability of being in poverty despite not being in poverty four year prior is at most 0.175 (0.192) in the first (second) SIPP panel. For the second SIPP panel, this provides a useful upper bound on the transition rate into poverty around the time of the Great Recession.

In Panel III the bounds are more informative. Thus, the assumption of uniform errors has some identifying power. Under this assumption, the probability of escaping poverty is at least $0.130(0.142)$ in the first (second) SIPP panel. The probability of remaining out of poverty is at least $0.882(0.865)$ in the first (second) SIPP panel. Conversely, the probability of being in poverty despite not being in poverty four year prior is at most $0.118(0.135)$ in the first (second) SIPP panel. This is about a six percentage point decline relative to Panel II. Finally, in both panels we are able to rule out the possibility (at the $90 \%$ confidence level) that no households move into poverty over the four year period; the probability of transitioning from out of poverty in the initial period to in poverty in the terminal period is at least 0.005 (0.020) in the first (second) SIPP panel.

Panels IV and $\mathrm{V}$ in Table 2 add the assumption that misclassification is only in the upward direction (Assumption 3). This assumption has no identifying power on the transition

\footnotetext{
${ }^{25}$ Throughout the discussion of the results, unless otherwise noted, we focus on the point estimates for simplicity. The confidence intervals are generally not much wider than the point estimates of the bounds.
} 
probabilities for households above the poverty line in the initial period. However, it is useful in tightening the bounds on the transition probabilities for households in poverty in the initial period. With arbitrary and uni-directional misclassification (Assumptions $2(i)$ and 3), bounds on the probability of remaining in poverty four years later are $[0.243,1.000]$ in the first SIPP panel and $[0.258,1.000]$ in the second SIPP panel. Under uniform and uni-directional misclassification (Assumptions 2(ii) and 3), bounds on the probability of remaining in poverty four years later are further tightened to $[0.315,0.870]$ in the first SIPP panel and $[0.331,0.858]$ in the second SIPP panel. While the assumptions of uniform and uni-directional misclassification certainly tighten the bounds, the width of the bounds under the assumption of a $10 \%$ misclassification rate makes it clear than even relatively small amounts of misclassification add considerable uncertainty to estimates of poverty mobility in a (relatively) low poverty environment. That said, one still learns that the four-year poverty persistence rate is at least 0.315 (0.331) in the first (second) SIPP panel under the strictest assumptions (Panel V).

In all cases, there is little advantage to pooling the panels as the bounds do not substantively differ across the two panels.

Level Set Restrictions Table 3 imposes different combinations of Assumptions 2,7. For the separate SIPP panels, level set restrictions are based on the age of the household head in the initial period. For the pooled panels, level set restrictions (Assumption 5) based on the age of the household head are imposed within each panel and stationarity (Assumption 7) is imposed across the panels. In Panel I, the level set restrictions are not combined with shape restrictions (Assumption 4). In Panel II, Assumption 4 is added to the level set restrictions. Assumption 4 corresponds to the restriction that households are more likely to maintain the same poverty status over the four-year period than change status. With each panel, we present results based on different types of misclassification errors based on Assumptions 2 -3.

Several findings stand out. First, under arbitrary and independent misclassification errors (Assumptions 2( $i)$ and 6), Panels IA and IIA reveal that the level set and shape restrictions have little identifying power. There is some tightening of the lower bounds relative to Panel II in Table 2, but it is modest. 
Second, under uniform and independent misclassification errors (Assumptions 2(ii) and 6), Panels IB and IIB reveal that the level set and shape restrictions have some identifying power. For example, bounds on the probability of remaining in poverty over the fouryear period in the first SIPP panel under uniform errors alone are $[0.026,0.870]$ (Table 2, Panel III), under level set restrictions with independent errors are [0.099, 0.822] (Table 3, Panel IB), and under level set and shape restrictions with independent errors is $[0.175,0.822]$ (Table 3, Panel IIB). In addition, if we utilize the pooled panels and impose the stationarity assumption, the bounds are further tightened to [0.196, 0.823] (Table 3, Panel IIB). Under these assumptions, at least 1 in 5 impoverished households in the initial period remain in poverty four years later. Similarly, bounds on the probability of escaping poverty over the four-year period in the first SIPP panel under uniform errors alone are [0.130, 0.974] (Table 2, Panel III), under level set restrictions with independent errors are [0.178, 0.901] (Table 3, Panel IB), and under level set and shape restrictions with independent errors is [0.178, 0.825] (Table 3, Panel IIB). In addition, if we utilize the pooled panels and impose the stationarity assumption, the bounds are further tightened to [0.177, 0.804] (Table 3, Panel IIB). Thus, we also find under these assumptions that at least 1 in 5 impoverished households in the initial period are out of poverty four years later.

Third, adding the assumption of uni-directional misclassification errors has additional identifying power on the transition probabilities for households below the poverty line in the initial period. Now the bounds on the probability of remaining in poverty over the fouryear period in the first SIPP Panel are [0.345, 0.822] (Table 3, Panel IIC), implying that at least 3 in 10 impoverished households in the initial period remain in poverty four years later. Finally, adding the stationarity assumption modestly tightens the bounds further; bounds on the probability of remaining in poverty over the four-year period under uniform, independent, and uni-directional errors are [0.357, 0.823] (Table 3, Panels IIC). Furthermore, under the strongest set of assumptions (Table 3, Panel IIC, using the pooled panels), we obtain bounds on the probability of escaping poverty four years later to be $[0.177,0.643]$ and on the probability of entering into poverty to be $[0.030,0.115]$. Knowledge of the minimum probability of escaping poverty and maximum probability of entering into poverty are useful policy parameters and the bounds appear narrow enough to be useful. 
Monotonicity Restriction Table 4 is similar to Table 3, but adds Assumption 8, The monotonicity restriction requires upward mobility to be weakly increasing in the household head's education level in the initial period. The monotonicity assumption has some identifying power. First, under arbitrary and independent misclassification errors (Assumptions $2(i)$ and 6), Panels IA and IIA reveal wide bounds, but now exclude the endpoints of zero and one in some instances.

Second, under our strongest set of assumptions, bounds on the probability of remaining in poverty over the four-year period are [0.357, 0.723] (Table 4, Panel IIC, using the pooled panels), in contrast to bounds of $[0.357,0.823]$ without monotonicity (Table 3, Panels IIC, using the pooled panels). Similarly, monotonicity tightens the bounds on the probability of escaping poverty over the four-year period from $[0.177,0.643]$ to $[0.277,0.643]$. Finally, monotonicity tightens the bounds on the probability of entering poverty over the four-year period from $[0.030,0.115]$ to $[0.032,0.113]$.

Sensitivity to $Q$ To explore the sensitivity of the bounds to the choice of $Q$, we re-estimate the bounds for several values of $Q$ ranging from 0 to 0.20 . For the sake of computational time, we focus on the point estimates of the bounds, not the confidence regions. Select results using the pooled sample are presented in Figures E1-E3 in Appendix E. There are three primary takeaways. First, the bounds are much wider for the transition probabilities for households in poverty in the initial period since only about $10 \%$ of the sample reports being in poverty in any period. Thus, small amount of measurement error can be extremely consequential when estimating poverty transitions in (relatively) low poverty environments. Second, the restrictions have more identifying power for these same transition probabilities. Consequently, despite the width of the bounds on these parameters, perhaps reasonable restrictions can be used to make the bounds markedly tighter. Finally, the lower (upper) bound for the probability of remaining in (escaping from) poverty is less sensitive to $Q$ in an absolute sense than the upper (lower) bound under our strictest set of restrictions. For instance, if we increase $Q$ from 0.10 to 0.20 , the lower bound on the probability of remaining in poverty over the sample period falls only from 0.36 to 0.28 . The corresponding change in the upper bound on the probability of escaping from poverty increases from 0.64 to 0.72 . 
However, the same increase in $Q$ raises the upper bound on the probability of remaining in poverty over the sample period from 0.72 to 0.98 ; the corresponding change in the lower bound on the probability of escaping from poverty declines from 0.28 to 0.02 . Thus, changes in $Q$ does not have the same impact on facets of the information that can be learned from our partial identification approach.

\subsubsection{Tercile Transition Matrix}

Results for the $3 \times 3$ tercile transition matrix based on OECD equivalized household income are presented in Tables 5-7. These tables are analogous to Tables 2-4 except we no longer consider the assumption of uni-directional misclassification since now any upward misclassification must induce downward misclassification as well. Results based on alternative equivalence scales are reported in Appendix E, Tables E1-E8.

Under the baseline assumption of Classification-Preserving Measurement Error (Table 5, Panel I) the conditional staying probabilities in the first (second) SIPP panel are 0.683, 0.533 , and $0.692(0.685,0.538$, and 0.685$)$ for terciles 1,2 , and 3 , respectively. Thus, the observed four-year conditional staying probabilities do not vary much across the two panels. Furthermore, we find that the probability of observing larger movements in the income distribution are less likely than smaller movements. For example, pooling the two panels together, the probability of moving from the first to second tercile is 0.245 and the first to third tercile is 0.071 . Similarly, the probability of moving from the third to second tercile is 0.217 and the third to first tercile is 0.095 .

Misclassification Assumptions Panels II and III in Table 5 allow for misclassification, but impose Assumption 2(i) and 2(ii), respectively. The assumed maximum misclassification rate is $20 \%(Q=0.20)$. The rationale for this choice is discussed in Appendix D; we also explore sensitivity to this choice below. Under arbitrary misclassification (Assumption 2(i)], the width of the bounds is $0.6(=K Q)$ unless the bounds include one of the boundaries. Under uniform misclassification (Assumption 2(ii)], the width is $0.4(=2 Q)$ unless the bounds hit one of the boundaries. Thus, the bounds are guaranteed to be at least somewhat informative only in the latter case. Uniform misclassification is reasonable if misclassification 
is equally likely in the upward and downward directions. With mean-reverting measurement error in income, this may be plausible.

In the first SIPP panel, we find that the bounds on the conditional staying probabilities are $[0.383,0.983],[0.233,0.833]$, and $[0.392,0.992]$ across terciles 1,2 , and 3 under arbitrary misclassification. The bounds tighten to [0.483, 0.883], [0.333, 0.733], and [0.492, 0.892] under uniform misclassification. Similar bounds arise in the second and pooled panels. Bounds on the off-diagonal elements, while generally lower as one moves further from the diagonal, cannot rule out the possibility that large movements in the income distribution are more likely than smaller movements (conditional on changing terciles). Moreover, bounds on the off-diagonal provide a useful upper bound on the probability of large income changes. For example, the probability of moving from tercile 1 to tercile 3 (tercile 3 to tercile 1 ) in the first SIPP panel under uniform misclassification is no greater than $0.271(0.287)$.

Level Set Restrictions Table 6 allows for misclassification, but imposes different combinations of Assumptions 2,7$]^{26}$ Because of the similarity of the results across the two SIPP panels in Table 5, we focus on the results for the pooled sample where the stationarity restriction (Assumption 7) is imposed. In Panel I, the level set restrictions are not combined with shape restrictions (Assumption 4). In Panel II, shape restrictions are imposed on top of the level set restrictions. This assumption corresponds to the restriction that households are more likely to make smaller movements in the income distribution than larger movements.

Several findings stand out. First, under arbitrary and independent misclassification errors (Assumptions 2( $i)$ and 6), Panels IA and IIA reveal that the level set restrictions have some identifying power. The shape restrictions do not add new information. As stated previously, the bounds under arbitrary errors in Table 5 have a width of 0.6 unless the boundary comes into play. After imposing the level set restrictions, the width of the bounds on the conditional staying probabilities falls to around 0.5. Thus, while still wide, there is some information in the level set restrictions. Second, under uniform and independent misclassification errors (Assumptions 2(ii) and 6), Panels IB and IIB reveal that the level set restrictions continue to have some identifying power. The shape restrictions continue to add no new information.

\footnotetext{
${ }^{26}$ For brevity, not all combinations are presented. Full results are available upon request.
} 
The bounds under uniform errors in Table 5 have a width of 0.4 unless the boundary comes into play. After imposing the level set restrictions, the width of the bounds on the conditional staying probabilities falls to around 0.3 . For example, bounds on the probability of remaining in the bottom tercile over the four-year period in the pooled sample under uniform errors alone are [0.485, 0.885] (Table 5, Panel III), but under level set restrictions with independent errors are [0.530,0.817] (Table 6, Panel IB); corresponding bounds on the probability of remaining in the top tercile tighten from [0.488, 0.888] (Table 5, Panel III) to [0.531, 0.850] (Table 6, Panel IB). Finally, bounds on the immediate off-diagonal elements exclude zero under the assumption of uniform and independent errors with the level set restrictions. Thus, we can rule out the possibility of no mobility to adjacent partitions.

Monotonicity Restriction Table 7 adds the monotonicity assumption. In general, the monotonicity assumption has only modest identifying power under either arbitrary or uniform, independent errors. For instance, the bounds on the probability of remaining in the bottom tercile across the initial and terminal periods in the pooled sample tighten from $[0.445,0.900]$ to $[0.445,0.893]$ under arbitrary, independent errors (Panel IA in Table 6 and 7). The corresponding bounds for the top tercile tighten from $[0.531,0.850]$ to $[0.531,0.820]$. However, the monotonicity assumption does help tighten the bounds on the probabilities of large income jumps. Specifically, the bounds on the probability of moving from the bottom to the top tercile in the pooled sample tighten from $[0.000,0.221]$ to $[0.000,0.129]$ under uniform, independent errors (Panel IIB in Table 6 and 7). The corresponding bounds on the probability of moving from the top to the bottom tercile tighten from $[0.000,0.274]$ to $[0.000,0.201]$. Knowledge of the maximum probability of large changes in position within the income distribution are useful policy parameters and, as with the poverty transition matrices, the bounds appear narrow enough to be useful.

Summary Mobility Measures Bounds on the summary mobility measures are reported in Table 8.27 Generally speaking, three conclusions can be drawn by this exercise. First, relative to the baseline assumption of Classification-Preserving Measurement Error, one can

\footnotetext{
${ }^{27}$ For brevity, Table 8 displays only the $90 \%$ confidence intervals and not the point estimates of the bounds. In addition, only the results for the individual panels are provided. All results are available upon request.
} 
assess the dramatic increase in uncertainty once misclassification rates of $20 \%$ are allowed. For example, the $90 \%$ confidence interval for the measure of upward mobility in the first SIPP panel is $[0.458,0.494]$ under classification-preserving measurement error. Under the assumption of arbitrary errors (with $Q=0.20$ ), the confidence interval is [0.012,0.940]. Second, our strictest set of assumptions - uniform, independent errors under level set, shape, and monotonicity restrictions - can tighten these bounds. Under these assumptions, the $90 \%$ confidence interval for the measure of upward mobility in the first SIPP panel is tightened to $[0.215,0.732]$. Finally, the bounds differ very little across the two SIPP panels. Thus, allowing for misclassification, there is no evidence that mobility changed across the two panels.

Sensitivity to $Q$ To explore the sensitivity of the bounds to the choice of $Q$, we reestimate the bounds for several values of $Q$ ranging from 0 to 0.40 . Point estimates of the bounds under select combinations of restrictions using the pooled sample are presented in Figures E4-E5 in Appendix E. There are three primary insights. First, the bounds are essentially linear in $Q$ except under the strictest set of restrictions shown (Assumptions 2(ii), 6. 5, 7, and 8). In these cases, the assumption of uniform misclassification (Assumption 2(ii) has significant identifying power over the assumption of arbitrary misclassification (Assumption 2(i) ); adding the level set and stationarity restrictions (Assumptions 5 and 7 ) further shrinks many of the bounds. Second, upon adding the monotonicity restriction to the previous assumptions, we find that the bounds may exclude the transition probability observed in the data. For example, the bounds for $p_{13}^{*}$ when $Q=0.10$ are $[0.00,0.05]$ despite the fact that the observed probability, $p_{13}$, is 0.07 . This arises, in this instance, because the monontonicity restriction assumes that $p_{13}^{*}$ is increasing in the monotone instrument, $u$ (education). However, under some combinations of other restrictions, $p_{13}^{*}$ is smallest for the highest education group and is, in fact, less than the observed probability, $p_{13}$, in the full sample. This may provide a reason to be skeptical about either the monotonicity restriction or the low value of $Q$. For all $Q \geq 0.20$, the bounds even under the strictest set of restrictions include the observed probability.

Finally, upon adding the monotonicity restriction to the previous assumptions, we also 
find that the bounds may be non-monotonic in $Q$. For example, the bounds for $p_{13}^{*}$ are $[0.00,0.05]$ when $Q=0.10$ and $[0.00,0.02]$ when $Q=0.15$. This can arise due to our implementation of the level set restrictions. To see this, consider the following simple example. Suppose the level set variable, $x$, takes on two values, $x_{1}$ and $x_{2}$. The level set restriction assumes $p_{k l}^{*}\left(x_{1}\right)=p_{k l}^{*}\left(x_{2}\right)$. Further suppose the bounds $p_{k l}^{*}\left(x_{j}\right), j=1,2$, under some set of assumptions and a particular $Q$ are $[0.15,0.25]$ and $[0.30,0.40]$, respectively. Because the bounds do not overlap, $p_{k l}^{*}\left(x_{1}\right) \neq p_{k l}^{*}\left(x_{2}\right)$ under the imposed set of assumptions. In such a case, we do not impose the level set restriction, we leave the bounds for $p_{k l}^{*}\left(x_{j}\right)$, $j=1,2$, unchanged and proceed. Now, if $Q$ is increased but the remaining assumptions are maintained, suppose the bounds for $p_{k l}^{*}\left(x_{j}\right), j=1,2$, widen to [0.10,0.30] and [0.25, 0.45], respectively. The level restrictions now yield identical, tighter bounds on $p_{k l}^{*}\left(x_{j}\right), j=1,2$, given by $[0.25,0.30]$. Thus, the increase in $Q$ allows the level set restrictions to now be plausible, leading to significantly tighter bounds. The tighter bounds reflect not just the higher $Q$, but also the ability to impose the level set restrictions.

\section{Conclusion}

That self-reported income contains complex, nonclassical measurement error is a well-established fact. That administrative data on income is imperfect is also relatively incontrovertible. As such, addressing measurement error in the study of income mobility should no longer be optional. To that end, several recent attempts to address measurement error have been put forth. Here, we offer a new and complementary approach based on the partial identification of transition matrices.

Among others, our approach has the advantage of transparency, as the assumptions used to tighten the bounds are easily understood and may be imposed in any combination depending on the particular context and the beliefs of the researcher. Moreover, our approach only requires data at two points in time. Finally, our approach extends easily to applications

other than income. The primary drawback to our approach is the lack of point identification. Consequently, our approach should be viewed as a complement to existing approaches that produce point estimates under more stringent (or, at least, alternative) identifying assump- 
tions. Using data from the SIPP, we show that relatively small amounts of measurement error leads to bounds that can be quite wide in the absence of other information or restrictions. However, the restrictions we consider contain significant identifying power. We are hopeful that future work will consider additional restrictions that may be used to further tighten the bounds on transition probabilities, as well as bounds on additional summary measures of mobility derived from the transition matrix.

\section{References}

[1] Andrews, D.W.K. (2000), "Inconsistency of the Bootstrap When a Parameter is on the Boundary of the Parameter Space," Econometrica, 68, 399-405.

[2] Andrews, D.W.K. and P. Guggenberger (2009), "Validity of Subsampling and 'Plug-In Asymptotic' Inference for Parameters Defined by Moment Inequalities," Econometric Theory , 25, 669-709.

[3] Atkinson, A.B. and F. Bourguignon (2015), "Introduction: Income Distribution Today," in A. B. Atkinson and F. Bourguignon (eds.) Handbook of Income Distribution, Vol. 2, Amsterdam: Elsevier-North Holland, xvii-lxiv.

[4] Bontemps, C. and T. Magnac (2017), "Set Identification, Moment Restrictions and Inference," Annual Review of Economics, 9, 103-129.

[5] Bound, J., C. Brown, and N. Mathiowitz (2001), "Measurement Error in Survey Data" in J. J. Heckman and E. Leamer (eds.) Handbook of Econometrics, New York: Elsevier Science, 3707-3745.

[6] Bradbury, K. (2016), "Levels and Trends in the Income Mobility of U.S. Families, 19772012," Working Paper 16-8, Federal Reserve Bank of Boston, Boston, MA.

[7] Burkhauser, R. and K. Couch (2009), "Intragenerational Inequality and Intertemporal Mobility," in W. Salverda, B. Nolan, and T.M. Smeeding (eds.) Oxford Handbook of Economic Inequality, Oxford: Oxford University Press, 522-548. 
[8] Canay, I. and A.M. Shaikh (2017). "Practical and Theoretical Advances in Inference for Partially Identified Models," in B. Honor, A. Pakes, M. Piazzesi, and L. Samuelson (eds.) Advances in Economics and Econometrics: Eleventh World Congress (Econometric Society Monographs) (Volume II), 271-306.

[9] Chernozhukov, V., H. Hong, and E. Tamer (2007), "Estimation and Confidence Regions for Parameter Sets in Econometric Models," Econometrica, 75, 1243-1284.

[10] Chernozhukov, V., S. Lee, and A.M. Rosen (2013), "Intersection Bounds: Estimation and Inference," Econometrica, 81, 667-737.

[11] Chetverikov, D., A. Santos, and A.M. Shaikh (2018), "The Econometrics of Shape Restrictions," Annual Review of Economics, 10, 31-63.

[12] Chiappori, P.A. (2016), "Equivalence Versus Indifference Scales," Economic Journal, $126,523-545$.

[13] Dang, H, P. Lanjouw, J. Luoto, and D. McKenzie (2014), "Using Repeated CrossSections to Explore Movements Into and Out of Poverty," Journal of Development Economics, 107, 112-128.

[14] Dragoset, L.M. and G.S. Fields (2006), "U.S. Earnings Mobility: Comparing SurveyBased and Administrative-Based Estimates," Working Paper 2006-55, ECINEQ.

[15] Glewwe, P. (2012), "How Much of Observed Economic Mobility is Measurement Error? IV Methods to Reduce Measurement Error Bias, with an Application to Vietnam," World Bank Economic Review, 26, 236-264.

[16] Glewwe, P. and H. Dang (2011), "Was Vietnam's Economic Growth in the 1990s ProPoor?" Economic Development and Cultural Change, 59, 583-608.

[17] Gottschalk, P. and M. Huynh (2010), "Are Earnings Inequality and Mobility Overstated? The Impact of Nonclassical Measurement Error," Review of Economics and Statistics, 92, 302-315. 
[18] Gundersen, C. and B. Kreider (2008), "Food Stamps and Food Insecurity: What Can Be Learned in the Presence of Nonclassical Measurement Error?" Journal of Human Resources, 43, 352-382.

[19] Gundersen, C. and B. Kreider (2009), "Bounding the Effects of Food Insecurity on Children's Health Outcomes," Journal of Health Economics, 28, 971-983.

[20] Heckman, J.J., J. Smith, and N. Clements (1997), "Making the Most Out of Programme Evaluations and Social Experiments: Accounting for Heterogeneity in Programme Impacts," Review of Economic Studies, 64, 487-535.

[21] Ho, K. and A.M. Rosen (2017), "Partial Identification in Applied Research: Benefits and Challenges," in B. Honore, A. Pakes, M. Piazzesi, L. Samuelson (eds.) Advances in Economics and Econometrics: Eleventh World Congress (Econometric Society Monographs) (Volume II), 307-359.

[22] Horowitz, J.L. (2001), "The Bootstrap," in J.J. Heckman and E.E. Leamer (eds.) Handbook of Econometrics, Vol. 5, Amsterdam, North-Holland.

[23] Horowitz, J.L. and C.F. Manski (1995), "Identification and Robustness with Contaminated and Corrupted Data," Econometrica, 63, 281-302.

[24] Imbens, G.W. and C.F. Manski (2004), "Confidence Intervals for Partially Identified Parameters," Econometrica, 72, 1845-1857.

[25] Jäntti, M. and S. Jenkins (2015), "Economic Mobility," in A. B. Atkinson and F. Bourguignon (eds.) Handbook of Income Distribution, Vol. 2, Amsterdam: Elsevier-North Holland, 807-935.

[26] Kapteyn, A. and J.Y. Ypma (2007), "Measurement Error and Misclassification: A Comparison of Survey and Administrative Data," Journal of Labor Economics, 25, 513551.

[27] Kopczuk, W., E. Saez, and J. Song (2010), "Earnings Inequality and Mobility in the United States: Evidence from Social Security Data Since 1937," Quarterly Journal of Economics, 125, 91-128. 
[28] Kreider, B. and J. V. Pepper (2007), "Disability and Employment: Reevaluating the Evidence in Light of Reporting Errors," Journal of the American Statistical Association, $102,432-441$.

[29] Kreider, B. and J.V. Pepper (2008), "Inferring Disability Status from Corrupt Data," Journal of Applied Econometrics, 23, 329-349.

[30] Kreider, B., J. V. Pepper, C. Gundersen, and D. Jolliffe (2012), "Identifying the Effects of SNAP (Food Stamps) on Child Health Outcomes When Participation Is Endogenous and Misreported," Journal of the American Statistical Association, 107, 958-975.

[31] Lechner, M. (1999), "Nonparametric Bounds on Employment and Income Effects of Continuous Vocational Training in East Germany," Econometrics Journal, 2, 1-28.

[32] Lee, N., G. Ridder, and J. Strauss (2017), "Estimation of Poverty Transition Matrices with Noisy Data," Journal of Applied Econometrics, 32, 37-55.

[33] Manski, C.F. (1990), "Nonparametric Bounds on Treatment Effects," American Economic Review, 80, 319-323.

[34] Manski, C.F. and J.V. Pepper (2000), "Monotone Instrumental Variables: With an Application to the Returns to Schooling," Econometrica, 68, 997-1010.

[35] Martínez-Muñoz, G. and A. Suáreza (2010), "Out-of-bag Estimation of the Optimal Sample Size in Bagging," Pattern Recognition, 43, 143-152.

[36] McGarry, K. (1995), "Measurement Error and Poverty Rates of Widows," Journal of Human Resources, 30, 113-134.

[37] Meyer, B.D., W.K.C. Mok, and J.X. Sullivan (2015), "Household Surveys in Crisis," Journal of Economic Perspectives, 29, 199-226.

[38] Molinari, F. (2008), "Partial Identification of Probability Distributions with Misclassified Data," Journal of Econometrics, 144, 81-117.

[39] OECD (1982), The OECD List of Social Indicators, Paris: OECD. 
[40] Pavlopoulos, D., R. Muffels, and J. K. Vermunt (2012), "How Real is Mobility Between Low Pay, High Pay and Non-Employment?" Journal of the Royal Statistical Society, $175,749-773$.

[41] Politis, D.N., J.P. Romano, and M. Wolf (1999), Subsampling, New York: SpringerVerlag.

[42] Rendtel, U., R. Langeheine, and R. Berntsen (1998), "The Estimation of Poverty Dynamics Using Different Measurements of Household Income," Review of Income and Wealth, 44, 81-98.

[43] Shorrocks, A.F. (1978), "The Measurement of Mobility," Econometrica, 46, 1013-1024.

[44] Tamer, E. (2010), "Partial Identification in Econometrics," Annual Review of Economics, 2, 167-195.

[45] Vikström, J., G. Ridder, and M. Weidner (2018), "Bounds on Treatment Effects on Transitions," Journal of Econometrics, 205, 448-469. 


\begin{tabular}{|c|c|c|c|c|c|c|c|c|}
\hline & \multicolumn{4}{|c|}{ 2004-2008 Panel } & \multicolumn{4}{|c|}{ 2008-2012 Panel } \\
\hline & \multicolumn{2}{|c|}{ Initial } & \multicolumn{2}{|c|}{ Terminal } & \multicolumn{2}{|c|}{ Initial } & \multicolumn{2}{|c|}{ Terminal } \\
\hline & Mean & SD & Mean & SD & Mean & SD & Mean & SD \\
\hline \multicolumn{9}{|l|}{ Household Income (Monthly) } \\
\hline Total Income & 5432 & 5481 & 5904 & 5768 & 6146 & 5875 & 6173 & 5985 \\
\hline Per Capita Income & 2233 & 2452 & 2427 & 2440 & 2605 & 2693 & 2600 & 2689 \\
\hline Equalized Income (OECD Scale) & 2720 & 2801 & 2937 & 2791 & 3145 & 3039 & 3121 & 3030 \\
\hline Equalized Income (Modified OECD Scale) & 3158 & 3168 & 3401 & 3172 & 3631 & 3413 & 3597 & 3402 \\
\hline Below Poverty Line (1 = Yes) & 0.118 & 0.323 & 0.107 & 0.309 & 0.126 & 0.332 & 0.126 & 0.332 \\
\hline \multicolumn{9}{|l|}{ Household Size } \\
\hline Total & 2.847 & 1.495 & 2.787 & 1.512 & 2.764 & 1.508 & 2.755 & 1.537 \\
\hline Number of Adults & 2.029 & 0.843 & 2.077 & 0.908 & 2.001 & 0.853 & 2.092 & 0.945 \\
\hline Number of Children Less Than 18 & 0.819 & 1.139 & 0.710 & 1.102 & 0.763 & 1.127 & 0.663 & 1.079 \\
\hline \multicolumn{9}{|l|}{ Age (Household Head) } \\
\hline 25-34 (1 = Yes) & 0.147 & 0.354 & 0.147 & 0.354 & 0.137 & 0.344 & 0.137 & 0.344 \\
\hline 35-44 (1 = Yes) & 0.276 & 0.447 & 0.276 & 0.447 & 0.240 & 0.427 & 0.240 & 0.427 \\
\hline 45-54 (1 = Yes) & 0.311 & 0.463 & 0.311 & 0.463 & 0.311 & 0.463 & 0.311 & 0.463 \\
\hline 55-65 (1 = Yes) & 0.266 & 0.442 & 0.266 & 0.442 & 0.312 & 0.463 & 0.312 & 0.463 \\
\hline \multicolumn{9}{|l|}{ Education (Household Head) } \\
\hline High School or Less (1 = Yes) & 0.346 & 0.476 & 0.346 & 0.476 & 0.321 & 0.467 & 0.321 & 0.467 \\
\hline Some College $(1=$ Yes $)$ & 0.367 & 0.482 & 0.367 & 0.482 & 0.354 & 0.478 & 0.354 & 0.478 \\
\hline Bachelor's Degree or More (1 = Yes) & 0.288 & 0.453 & 0.288 & 0.453 & 0.325 & 0.469 & 0.325 & 0.469 \\
\hline $\mathrm{N}$ & 783 & & 7834 & & 16006 & & 1600 & \\
\hline
\end{tabular}

Notes: Samples from the Survey of Income and Program Participation (SIPP). 
Table 2. Poverty Transition Matrices: Misclassification Assumptions.

2004-2008 Pane

I. Classification-Preserving Measurement Error

$\begin{array}{ccc} & \begin{array}{c}\text { Below } \\ \text { Poverty }\end{array} & \begin{array}{c}\text { Above } \\ \text { Poverty }\end{array} \\ \text { Below } & {[0.448,0.448]} & {[0.552,0.552]} \\ \text { Poverty } & (0.423,0.474) & (0.526,0.577) \\ \text { Above } & {[0.061,0.061]} & {[0.939,0.939]} \\ \text { Poverty } & (0.057,0.066) & (0.934,0.943)\end{array}$

II. Arbitrary Misclassification $(\mathrm{Q}=0.10)$

$\begin{array}{ccc}\text { Below } & \begin{array}{c}\text { Below } \\ \text { Poverty } \\ {[0.000,1.000]}\end{array} & \begin{array}{c}\text { Above } \\ \text { Poverty } \\ {[0.000,1.000]}\end{array} \\ \text { Poverty } & (0.000,1.000) & (0.000,1.000) \\ \text { Above } & {[0.000,0.175]} & {[0.825,1.000]} \\ \text { Poverty } & (0.000,0.179) & (0.821,1.000)\end{array}$

III. Uniform Misclassification $(\mathrm{Q}=0.10)$

$\begin{array}{ccc}\text { Below } & \begin{array}{c}\text { Above } \\ \text { Poverty }\end{array} & \begin{array}{c}\text { Poverty } \\ \text { Below }\end{array} \\ \text { Poverty } & (0.026,0.870] & {[0.130,0.974]} \\ \text { Above } & {[0.004,0.897)} & (0.103,0.996) \\ \text { Poverty } & (0.001,0.122) & {[0.882,0.995]} \\ \text { A } & (0.878,0.999)\end{array}$

\begin{tabular}{lcc} 
& 2008-2012 Panel & \\
\hline & $\begin{array}{c}\text { Below } \\
\text { Poverty }\end{array}$ & $\begin{array}{c}\text { Above } \\
\text { Poverty }\end{array}$ \\
Below & {$[0.462,0.462]$} & {$[0.538,0.538]$} \\
Poverty & $(0.444,0.480)$ & $(0.520,0.556)$ \\
Above & {$[0.077,0.077]$} & {$[0.923,0.923]$} \\
Poverty & $(0.074,0.081)$ & $(0.919,0.926)$
\end{tabular}

\begin{tabular}{|c|c|c|}
\hline \multicolumn{3}{|c|}{ Pooled Panels } \\
\hline & Below & Above \\
\hline & Poverty & Poverty \\
\hline Below & {$[0.457,0.457]$} & {$[0.543,0.543]$} \\
\hline Poverty & $(0.441,0.474)$ & $(0.526,0.559)$ \\
\hline Above & {$[0.072,0.072]$} & {$[0.928,0.928]$} \\
\hline Poverty & $(0.069,0.075)$ & $(0.925,0.931)$ \\
\hline
\end{tabular}

\begin{tabular}{lcc} 
Below & $\begin{array}{c}\text { Below } \\
\text { Poverty } \\
\text { Poverty }\end{array}$ & $\begin{array}{c}\text { Above } \\
\text { Poverty }\end{array}$ \\
Above & $(0.000,1.000]$ & {$[0.000,1.000]$} \\
Poverty & {$[0.000,1.000)$} & $(0.000,1.000)$ \\
\hline & $(0.000,0.195)$ & {$[0.808,1.000]$} \\
\end{tabular}

Below
Poverty
Above
Poverty

$$
\begin{gathered}
\text { Below } \\
\text { Poverty } \\
{[0.000,1.000]} \\
(0.000,1.000) \\
{[0.000,0.186]} \\
(0.000,0.189)
\end{gathered}
$$

\begin{tabular}{|c|c|c|}
\hline & $\begin{array}{c}\text { Below } \\
\text { Poverty }\end{array}$ & $\begin{array}{l}\text { Above } \\
\text { Poverty }\end{array}$ \\
\hline Below & {$[0.066,0.858]$} & {$[0.142,0.934]$} \\
\hline Poverty & $(0.047,0.875)$ & $(0.125,0.953)$ \\
\hline Above & {$[0.020,0.135]$} & {$[0.865,0.980]$} \\
\hline Poverty & $(0.017,0.138)$ & $(0.862,0.983)$ \\
\hline
\end{tabular}

$$
\begin{gathered}
\text { Above } \\
\text { Poverty } \\
{[0.000,1.000]} \\
(0.000,1.000) \\
{[0.814,1.000]} \\
(0.811,1.000)
\end{gathered}
$$

\begin{tabular}{|c|c|c|}
\hline & $\begin{array}{c}\text { Below } \\
\text { Poverty }\end{array}$ & $\begin{array}{c}\text { Above } \\
\text { Poverty }\end{array}$ \\
\hline Below & {$[0.243,1.000]$} & {$[0.000,0.757]$} \\
\hline Poverty & $(0.232,1.000)$ & $(0.000,0.768)$ \\
\hline Above & {$[0.000,0.175]$} & {$[0.825,1.000]$} \\
\hline Poverty & $(0.000,0.179)$ & $(0.821,1.000)$ \\
\hline
\end{tabular}

Below
Poverty
Above
Poverty

Below
Poverty
$[0.053,0.862]$
$(0.037,0.877)$
$[0.015,0.129]$
$(0.013,0.131)$

Above

Poverty

[0.138,0.947]

$(0.123,0.963)$

$[0.871,0.985]$

$(0.869,0.987)$

IV. Arbitrary, Uni-Directional Misclassification $(\mathrm{Q}=0.10)$

Below
Poverty
Above
Poverty

$\begin{array}{cc}\begin{array}{c}\text { Below } \\ \text { Poverty }\end{array} & \begin{array}{c}\text { Above } \\ \text { Poverty }\end{array} \\ {[0.258,1.000]} & {[0.000,0.742]} \\ (0.249,1.000) & (0.000,0.751) \\ {[0.000,0.192]} & {[0.808,1.000]} \\ (0.000,0.195) & (0.805,1.000)\end{array}$

Below
Poverty
Above
Poverty

\section{Above \\ Poverty \\ $[0.000,0.747]$ \\ $(0.000,0.755)$ \\ $[0.814,1.000]$ \\ $(0.811,1.000)$}

V. Uniform, Uni-Directional Misclassification $(\mathrm{Q}=0.10)$

$\begin{array}{ccc}\text { Below } & \begin{array}{c}\text { Below } \\ \text { Poverty }\end{array} & \begin{array}{c}\text { Above } \\ \text { Poverty }\end{array} \\ \text { Poverty } & {[0.315,0.870]} & {[0.130,0.685]} \\ \text { Above } & (0.301,0.897) & (0.103,0.699) \\ \text { Poverty } & {[0.005,0.118]} & {[0.882,0.995]} \\ & (0.001,0.122) & (0.878,0.999)\end{array}$

\begin{tabular}{ccc} 
Below & $\begin{array}{c}\text { Below } \\
\text { Poverty }\end{array}$ & $\begin{array}{c}\text { Above } \\
\text { Poverty }\end{array}$ \\
Poverty & {$[0.331,0.858]$} & {$[0.142,0.669]$} \\
Above & {$[0.320,0.875)$} & $(0.125,0.680)$ \\
Poverty & $(0.021,0.135]$ & {$[0.865,0.979]$} \\
\hline
\end{tabular}

\begin{tabular}{ccc} 
Below & $\begin{array}{c}\text { Below } \\
\text { Poverty }\end{array}$ & $\begin{array}{c}\text { Above } \\
\text { Poverty }\end{array}$ \\
Poverty & {$[0.326,0.862]$} & {$[0.138,0.674]$} \\
Above & {$[0.316,0.877)$} & $(0.123,0.684)$ \\
Poverty & $(0.016,0.129]$ & {$[0.871,0.984]$} \\
\hline
\end{tabular}

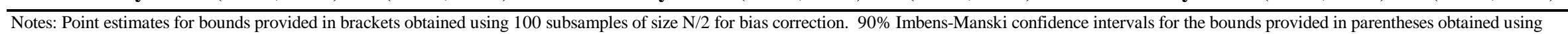
250 subsamples of size N/2. See text for further details. 
I. No Shape Restrictions

A. Arbitrary, Independent Misclassification $(\mathrm{Q}=0.10)$

\begin{tabular}{|c|c|c|c|c|c|}
\hline & Below & Above & & Below & Above \\
\hline & Poverty & Poverty & & Poverty & Poverty \\
\hline Below & {$[0.000,1.000]$} & {$[0.000,1.000]$} & Below & {$[0.000,1.000]$} & {$[0.000,1.000]$} \\
\hline Poverty & $(0.000,1.000)$ & $(0.000,1.000)$ & Poverty & $(0.000,1.000)$ & $(0.000,1.000)$ \\
\hline Above & {$[0.000,0.170]$} & {$[0.830,1.000]$} & Above & {$[0.000,0.184]$} & $0.816,1.000$ \\
\hline Poverty & $(0.000,0.175)$ & $(0.825,1.000)$ & Poverty & $(0.000,0.188)$ & $(0.812,1.00$ \\
\hline
\end{tabular}

Uniform, Independent Misclassification $(\mathrm{Q}=0.10)$

Poverty

$(0.000,0.188)$

$(0.812,1.000)$

$\begin{array}{cc}\begin{array}{c}\text { Below } \\ \text { Poverty }\end{array} & \begin{array}{c}\text { Above } \\ \text { Poverty }\end{array} \\ {[0.120,0.829]} & {[0.171,0.880]} \\ (0.099,0.853) & (0.147,0.901) \\ {[0.028,0.127]} & {[0.873,0.972]} \\ (0.023,0.131) & (0.869,0.977)\end{array}$

$\begin{array}{cc}\begin{array}{c}\text { Below } \\ \text { Poverty }\end{array} & \begin{array}{c}\text { Above } \\ \text { Poverty }\end{array} \\ {[0.099,0.822]} & {[0.178,0.901]} \\ (0.062,0.857) & (0.143,0.938) \\ {[0.009,0.115]} & {[0.885,0.991]} \\ (0.004,0.119) & (0.881,0.996)\end{array}$

Below

Below

Poverty

$(0.004,0.119)$

Poverty

Above

Poverty

C. Uniform, Independent, Uni-Directional Misclassification $(\mathrm{Q}=0.10)$

$\begin{array}{ccc}\text { Below } & \begin{array}{c}\text { Below } \\ \text { Poverty }\end{array} & \begin{array}{c}\text { Above } \\ \text { Poverty }\end{array} \\ \text { Poverty } & {[0.345,0.822]} & {[0.178,0.655]} \\ \text { Above } & (0.323,0.857) & (0.143,0.677) \\ \text { Poverty } & {[0.010,0.115]} & {[0.885,0.990]} \\ & (0.004,0.119) & (0.881,0.996)\end{array}$

Below
Poverty
Above
Poverty

Below
Poverty
$[0.363,0.829]$
$(0.349,0.853)$
$[0.030,0.127]$
$(0.025,0.131)$

Above

Poverty

$[0.171,0.637]$

$(0.147,0.651)$

$[0.873,0.970]$

$(0.869,0.975)$

Below

Poverty

Above

Poverty

Below

Poverty

Above

Poverty

\section{Below \\ Poverty \\ Above}

Poverty

A. Arbitrary, Independent Misclassification $(\mathrm{Q}=0.10)$

$\begin{array}{ccc}\text { Below } & \begin{array}{c}\text { Poverty } \\ \text { Below }\end{array} & \begin{array}{c}\text { Above } \\ \text { Poverty }\end{array} \\ \text { Poverty } & {[0.000,1.000]} & {[0.000,1.000]} \\ \text { Above } & {[0.000,1.000)} & (0.000,1.000) \\ \text { Poverty } & (0.000,0.170] & {[0.830,1.000]} \\ \end{array}$

B. Uniform, Independent Misclassification $(\mathrm{Q}=0.10)$

\begin{tabular}{|c|c|c|c|c|}
\hline & Below & Above & & Below \\
\hline & Poverty & Poverty & & Poverty \\
\hline Below & {$[0.175,0.822]$} & {$[0.178,0.825]$} & Below & {$[0.209,0.829]$} \\
\hline Poverty & $(0.143,0.857)$ & $(0.143,0.857)$ & Poverty & $(0.186,0.853)$ \\
\hline Above & {$[0.009,0.115]$} & {$[0.885,0.991]$} & Above & {$[0.028,0.127]$} \\
\hline Poverty & $(0.004,0.119)$ & $(0.881,0.996)$ & Poverty & $(0.023,0.131)$ \\
\hline
\end{tabular}

Below
Poverty
$[0.000,1.000]$
$(0.000,1.000)$
$[0.000,0.184]$
$(0.000,0.188)$

Below
Poverty
$[0.209,0.829]$
$(0.186,0.853)$
$[0.028,0.127]$
$(0.023,0.131)$

Above
Poverty
$[0.000,1.000]$
$(0.000,1.000)$
$[0.816,1.000]$
$(0.812,1.000)$

Above

Poverty

[0.171,0.791]

$(0.147,0.814)$

[0.873,0.972]

\section{Below}

Poverty

[0.000,1.000]

$(0.000,1.000)$

$[0.000,0.170]$

$(0.000,0.175)$

\section{Below}

Poverty

[0.123,0.823]

$(0.098,0.851)$

$[0.028,0.115]$

$(0.024,0.120)$

\section{Below}

Poverty

[0.357,0.823]

$(0.343,0.851)$

$[0.030,0.115]$

$(0.026,0.120)$
Above

Poverty

[0.000,1.000]

$(0.000,1.000)$

$[0.830,1.000]$

$(0.825,1.000)$

Above

Poverty

[0.177,0.877]

$(0.149,0.902)$

$[0.885,0.972]$

$(0.880,0.976)$

\section{Above}

Poverty

[0.177,0.643]

$(0.149,0.657)$

[0.885,0.970]

$(0.880,0.974)$

C. Uniform, Independent, Uni-Directional Misclassification $(\mathrm{Q}=0.10)$

\begin{tabular}{|c|c|c|c|}
\hline & Below & Above & \\
\hline & Poverty & Poverty & \\
\hline Below & {$[0.345,0.822]$} & {$[0.178,0.655]$} & Below \\
\hline Poverty & $(0.323,0.857)$ & $(0.143,0.677)$ & Poverty \\
\hline Above & {$[0.010,0.115]$} & {$[0.885,0.990]$} & Above \\
\hline Poverty & $(0.004,0.119)$ & $(0.881,0.996)$ & Poverty \\
\hline
\end{tabular}

\section{$(0.869,0.977)$}

Below

Poverty

$[0.363,0.829]$

$(0.349,0.853)$

$[0.030,0.127]$

$(0.025,0.131)$

$\begin{array}{cc}\text { Below } \\ \text { Below } & {[0.000,1.000]} \\ \text { Poverty } & (0.000,1.000) \\ \text { Above } & {[0.000,0.170]} \\ \text { Poverty } & (0.000,0.175)\end{array}$

\section{Above}

Poverty

$[0.000,1.000]$

$(0.000,1.000)$

[0.830,1.000]

$(0.825,1.000)$

Notes: Point estimates for bounds provided in brackets obtained using 100 subsamples of size N/2 for bias correction. 90\% Imbens-Manski confidence intervals for the bounds provided in parentheses obtained using

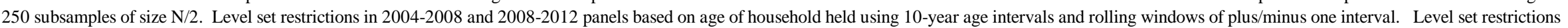
in pooled panel based on age of household held using 10-year age intervals and rolling windows of plus/minus one interval both within and across panels. See text for further details. 
I. No Shape Restrictions

$$
\text { 2004-2008 Panel }
$$

A. Arbitrary, Independent Misclassification $(\mathrm{Q}=0.10)$

\begin{tabular}{|c|c|c|c|c|c|}
\hline & Below & Above & & Below & Above \\
\hline & Poverty & Poverty & & Poverty & Poverty \\
\hline Belov & {$[0.020,0.981]$} & {$[0.019,0.980]$} & Below & {$[0.041,1.000]$} & {$[0.000,0.959]$} \\
\hline Poverty & $(0.007,1.000)$ & $(0.000,0.993)$ & Poverty & $(0.032,1.000)$ & $(0.000,0.968)$ \\
\hline Above & {$[0.000,0.167]$} & {$[0.833,1.000]$} & Above & {$[0.008,0.184]$} & {$[0.816,0.992$} \\
\hline
\end{tabular}

Uniform, Independent Misclassification $(\mathrm{Q}=0.10)$

Poverty

$(0.005,0.188)$

$(0.812,0.995)$

$\begin{array}{cccccc} & \begin{array}{c}\text { Below } \\ \text { Poverty }\end{array} & \begin{array}{c}\text { Above } \\ \text { Poverty }\end{array} & & \text { Below } & \begin{array}{c}\text { Above } \\ \text { Poverty }\end{array} \\ \text { Below } & {[0.099,0.729]} & {[0.271,0.901]} & \text { Below } & {[0.133,0.747]} & {[0.253,0.867]} \\ \text { Poverty } & (0.081,0.765) & (0.235,0.919) & \text { Poverty } & (0.119,0.772) & (0.228,0.881) \\ \text { Above } & {[0.019,0.111]} & {[0.889,0.981]} & \text { Above } & {[0.037,0.127]} & {[0.873,0.963]} \\ \text { Poverty } & (0.013,0.115) & (0.885,0.987) & \text { Poverty } & (0.032,0.131) & (0.869,0.968)\end{array}$

C. Uniform, Independent, Uni-Directional Misclassification $(\mathrm{Q}=0.10)$

\begin{tabular}{|c|c|c|}
\hline & & \\
\hline & Poverty & Poverty \\
\hline Below & {$[0.345,0.729]$} & {$[0.271,0.655]$} \\
\hline Poverty & $(0.323,0.765)$ & $(0.235,0.677)$ \\
\hline Above & {$[0.020,0.111]$} & {$[0.889,0.980]$} \\
\hline Poverty & $(0.014,0.115)$ & $(0.885,0.986)$ \\
\hline With Shape & tions & \\
\hline rbitrar & adent Misc & ation $(\mathrm{Q}$ \\
\hline & Below & Above \\
\hline & Poverty & Poverty \\
\hline Below & {$[0.025,0.981]$} & {$[0.019,0.975]$} \\
\hline Poverty & $(0.012,1.000)$ & $(0.000,0.988)$ \\
\hline Above & {$[0.000,0.167]$} & {$[0.833,1.000]$} \\
\hline Poverty & $(0.000,0.172)$ & $(0.828,1.000)$ \\
\hline
\end{tabular}

B. Uniform, Independent Misclassification $(\mathrm{Q}=0.10)$

Below
Poverty
Above
Poverty

Below
Poverty
$[0.363,0.747]$
$(0.349,0.772)$
$[0.040,0.127]$
$(0.035,0.131)$

Above

Poverty

[0.253,0.637]

$(0.228,0.651)$

$[0.873,0.960]$

$(0.869,0.965)$

Below
Poverty
Above
Poverty

Below

Poverty

Above

Poverty

Below
Poverty
Above
Poverty

$\begin{array}{cc}\begin{array}{c}\text { Below } \\ \text { Poverty }\end{array} & \begin{array}{c}\text { Above } \\ \text { Poverty }\end{array} \\ {[0.040,0.979]} & {[0.021,0.960]} \\ (0.031,1.000) & (0.000,0.969) \\ {[0.008,0.166]} & {[0.834,0.992]} \\ (0.005,0.171) & (0.829,0.995)\end{array}$

$(0.829,0.995)$

$\begin{array}{cc}\begin{array}{c}\text { Below } \\ \text { Poverty }\end{array} & \begin{array}{c}\text { Above } \\ \text { Poverty }\end{array} \\ {[0.138,0.723]} & {[0.277,0.862]} \\ (0.123,0.755) & (0.245,0.877) \\ {[0.040,0.107]} & {[0.893,0.960]} \\ (0.035,0.113) & (0.887,0.965)\end{array}$

\section{Above}

Poverty

$[0.277,0.643]$

$(0.245,0.657)$

$[0.887,0.968]$

$(0.881,0.973)$

A. Arbitrary, Independent Misclassification $(\mathrm{Q}=0.10)$

\begin{tabular}{|c|c|c|c|c|}
\hline & Below & Above & & \multirow{2}{*}{$\begin{array}{l}\text { Below } \\
\text { Poverty }\end{array}$} \\
\hline & Poverty & Poverty & & \\
\hline Below & {$[0.175,0.729]$} & {$[0.271,0.825]$} & Below & {$[0.209,0.747]$} \\
\hline Poverty & $(0.143,0.765)$ & $(0.235,0.857)$ & Poverty & $(0.186,0.772)$ \\
\hline Above & {$[0.019,0.111]$} & {$[0.889,0.981]$} & Above & {$[0.037,0.127]$} \\
\hline Poverty & $(0.013,0.115)$ & $(0.885,0.987)$ & Poverty & $(0.032,0.131)$ \\
\hline
\end{tabular}

$\begin{array}{cc}\begin{array}{c}\text { Below } \\ \text { Poverty }\end{array} & \begin{array}{c}\text { Above } \\ \text { Poverty }\end{array} \\ {[0.042,1.000]} & {[0.000,0.958]} \\ (0.033,1.000) & (0.000,0.967) \\ {[0.008,0.184]} & {[0.816,0.992]} \\ (0.005,0.188) & (0.812,0.995)\end{array}$

$\begin{array}{cc}\text { Below } \\ \text { Below } & {[0.041,0.979]} \\ \text { Poverty } & (0.033,1.000) \\ \text { Above } & {[0.008,0.166]} \\ \text { Poverty } & (0.005,0.171)\end{array}$

Above

Poverty

$[0.021,0.959]$

$(0.000,0.967)$

[0.834,0.992]

$(0.829,0.995)$

\section{Above}

Poverty

[0.253,0.791]

$(0.228,0.814)$

$[0.873,0.963]$

$(0.869,0.968)$

Below
Poverty
Above
Poverty

\section{Below}

Poverty

[0.196,0.723]

$(0.172,0.755)$

$[0.040,0.107]$

$(0.035,0.113)$

Above

Poverty

[0.277,0.804]

$(0.245,0.828)$

$[0.893,0.960]$

$(0.887,0.965)$

C. Uniform, Independent, Uni-Directional Misclassification $(Q=0.10)$

$\begin{array}{cccc} & \begin{array}{c}\text { Below } \\ \text { Poverty }\end{array} & \begin{array}{c}\text { Above } \\ \text { Poverty }\end{array} & \\ \text { Below } & {[0.345,0.729]} & {[0.271,0.655]} & \text { Below } \\ \text { Poverty } & (0.323,0.765) & (0.235,0.677) & \text { Poverty } \\ \text { Above } & {[0.020,0.111]} & {[0.889,0.980]} & \begin{array}{c}\text { Above } \\ \text { Poverty }\end{array} \\ \text { Poverty }\end{array}$

Below
Poverty
$[0.363,0.747]$
$(0.349,0.772)$
$[0.040,0.127]$
$(0.035,0.131)$

\section{Above}

Poverty

[0.253,0.637]

$(0.228,0.651)$

$[0.873,0.960]$

$(0.869,0.965)$

Below
Poverty
Above
Poverty

\begin{tabular}{cc}
$\begin{array}{c}\text { Below } \\
\text { Poverty }\end{array}$ & $\begin{array}{c}\text { Above } \\
\text { Poverty }\end{array}$ \\
{$[0.357,0.723]$} & {$[0.277,0.643]$} \\
$(0.343,0.755)$ & $(0.245,0.657)$ \\
{$[0.032,0.113]$} & {$[0.887,0.968]$} \\
$(0.027,0.119)$ & $(0.881,0.973)$ \\
\hline
\end{tabular}

Notes: Point estimates for bounds provided in brackets obtained using 100 subsamples of size N/2 for bias correction. $90 \%$ Imbens-Manski confidence intervals for the bounds provided in parentheses obtained using

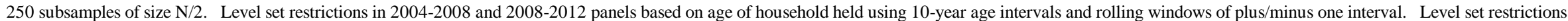

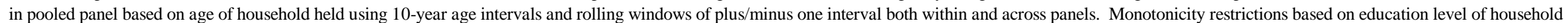
held using three categories (high school degree and below, some college, and four-year college degree or more). See text for further details. 
Table 5. Tercile Transition Matrices: Misclassification Assumptions.

\section{4-2008 Panel}

I. Classification-Preserving Measurement Error
$1 \quad[0.683,0.683] \quad[0.246,0.246] \quad[0.071,0.071]$

$2 \quad[0.231,0.231] \quad[0.533,0.533] \quad[0.236,0.236]$ $\begin{array}{lll}(0.219,0.243) \quad(0.519,0.546) & (0.226,0.246)\end{array}$

$3 \quad[0.087,0.087] \quad[0.221,0.221] \quad[0.692,0.692]$ $(0.078,0.095) \quad(0.210,0.232) \quad(0.682,0.703)$

II. Arbitrary Misclassification $(\mathrm{Q}=0.20)$

3

$1 \quad[0.383,0.983] \quad[0.000,0.546] \quad[0.000,0.371]$ $\begin{array}{llll}(0.373,0.992) & (0.000,0.556) & (0.000,0.378)\end{array}$

$2 \quad[0.000,0.531] \quad[0.233,0.833] \quad[0.000,0.536]$ $\begin{array}{llll}(0.000,0.540) & (0.222,0.843) & (0.000,0.544)\end{array}$

$3 \quad[0.000,0.387] \quad[0.000,0.521] \quad[0.392,0.992]$ $\begin{array}{lll}(0.000,0.393) & (0.000,0.529) & (0.384,1.000)\end{array}$

III. Uniform Misclassification $(\mathrm{Q}=0.20)$$$
2
$$

3

$1 \quad[0.483,0.883] \quad[0.046,0.446] \quad[0.000,0.271]$ $\begin{array}{llll}(0.473,0.892) & (0.037,0.456) & (0.000,0.278)\end{array}$

$2 \quad[0.031,0.431] \quad[0.333,0.733] \quad[0.036,0.436]$ $\begin{array}{llll}(0.022,0.440) & (0.322,0.743) & (0.028,0.444)\end{array}$

$3 \quad[0.000,0.287] \quad[0.021,0.421] \quad[0.492,0.892]$ $(0.000,0.293) \quad(0.013,0.429) \quad(0.484,0.900)$ $\begin{array}{llll}(0.670,0.695) & (0.234,0.258) & (0.063,0.079)\end{array}$
1

$1 \quad[0.685,0.685] \quad[0.242,0.242] \quad[0.073,0.073]$ $\begin{array}{llll}(0.678,0.693) & (0.234,0.250) & (0.067,0.079)\end{array}$

$2 \quad[0.220,0.220] \quad[0.538,0.538] \quad[0.242,0.242]$ $\begin{array}{llll}(0.212,0.228) & (0.529,0.546) & (0.234,0.250)\end{array}$

$3[0.095,0.095] \quad[0.220,0.220] \quad[0.685,0.685]$ $\begin{array}{lll}(0.089,0.101) & (0.213,0.228) \quad(0.677,0.693)\end{array}$

1

$1 \quad[0.385,0.985] \quad[0.000,0.542] \quad[0.000,0.373]$ $\begin{array}{llll}(0.380,0.991) & (0.000,0.548) & (0.000,0.377)\end{array}$

$2[0.000,0.520] \quad[0.238,0.838] \quad[0.000,0.542]$ $\begin{array}{lll}(0.000,0.526) & (0.231,0.844) & (0.000,0.548)\end{array}$

$3[0.000,0.395] \quad[0.000,0.520] \quad[0.385,0.985]$ $\begin{array}{lll}(0.000,0.400) \quad(0.000,0.526) & (0.379,0.991)\end{array}$
$1 \quad[0.485,0.885] \quad[0.042,0.442] \quad[0.000,0.273]$ $\begin{array}{llll}(0.480,0.891) & (0.036,0.448) & (0.000,0.277)\end{array}$

$2[0.020,0.420] \quad[0.338,0.738] \quad[0.042,0.442]$ $\begin{array}{llll}(0.014,0.426) & (0.331,0.744) & (0.036,0.448)\end{array}$

$\begin{array}{lllll}3 & {[0.000,0.295]} & {[0.020,0.420]} & {[0.485,0.885}\end{array}$ $\begin{array}{lll}(0.000,0.300) & (0.014,0.426) & (0.479,0.891)\end{array}$
Pooled Panels

$\begin{array}{llll}1 & 2 & 3\end{array}$

$1 \quad[0.685,0.685] \quad[0.245,0.245] \quad[0.071,0.071]$ $\begin{array}{lll}(0.678,0.691) & (0.239,0.252) & (0.067,0.076)\end{array}$

$2 \quad[0.220,0.220] \quad[0.538,0.538] \quad[0.240,0.240]$ $\begin{array}{llll}(0.214,0.226) & (0.531,0.545) & (0.234,0.247)\end{array}$

$3 \quad[0.095,0.095] \quad[0.217,0.217] \quad[0.688,0.688]$ $\begin{array}{lll}(0.090,0.100) & (0.211,0.223) \quad(0.682,0.694)\end{array}$

\section{3}

$1 \quad[0.385,0.985] \quad[0.000,0.545] \quad[0.000,0.371]$ $\begin{array}{lll}(0.380,0.990) & (0.000,0.551) & (0.000,0.375)\end{array}$

$2 \quad[0.000,0.520] \quad[0.238,0.838] \quad[0.000,0.540]$ $\begin{array}{llll}(0.000,0.525) & (0.232,0.843) & (0.000,0.545)\end{array}$

$3 \quad[0.000,0.395] \quad[0.000,0.517] \quad[0.388,0.988]$ $(0.000,0.399) \quad(0.000,0.521) \quad(0.383,0.993)$ bounds provided in parentheses obtained using 250 subsamples of size N/2. See text for further details. 
I. No Shape Restrictions

A. Arbitrary, Independent Misclassification $(Q=0.20)$ 3

$1 \quad[0.435,0.947] \quad[0.000,0.503] \quad[0.000,0.339]$ $\begin{array}{lll}(0.418,0.964) \quad(0.000,0.518) & (0.000,0.348)\end{array}$

$2 \quad[0.000,0.515] \quad[0.261,0.814] \quad[0.000,0.516]$ $\begin{array}{lll}(0.000,0.528) & (0.244,0.830) & (0.000,0.531)\end{array}$

$3 \quad[0.000,0.336] \quad[0.000,0.462] \quad[0.467,0.979]$ $\begin{array}{lll}(0.000,0.346) & (0.000,0.477) \quad(0.452,0.993)\end{array}$

B. Uniform, Independent Misclassification $(Q=0.20)$
$1 \quad[0.527,0.852] \quad[0.075,0.411] \quad[0.000,0.247]$ $\begin{array}{llll}(0.510,0.868) & (0.060,0.426) & (0.000,0.256)\end{array}$

$2 \quad[0.050,0.416] \quad[0.359,0.712] \quad[0.036,0.421]$ $\begin{array}{lll}(0.035,0.428) & (0.343,0.728) & (0.028,0.434)\end{array}$

$3 \quad[0.000,0.247] \quad[0.025,0.374] \quad[0.555,0.868]$ $\begin{array}{lll}(0.000,0.257) & (0.013,0.388) & (0.541,0.885)\end{array}$
1

$[0.422,0.975] \quad[0.000,0.512] \quad[0.000,0.347]$ $\begin{array}{lll}(0.414,0.985) & (0.000,0.520) & (0.000,0.352)\end{array}$

$2[0.000,0.507] \quad[0.256,0.829] \quad[0.000,0.528]$ $\begin{array}{lll}(0.000,0.517) & (0.244,0.840) & (0.000,0.537)\end{array}$

$3[0.000,0.379] \quad[0.000,0.483] \quad[0.421,0.951]$ $(0.000,0.386) \quad(0.000,0.494) \quad(0.410,0.960)$
II. With Shape Restrictions

A. Arbitrary, Independent Misclassification $(Q=0.20)$$$
2
$$

$1 \quad[0.435,0.947] \quad[0.000,0.503] \quad[0.000,0.339]$ $\begin{array}{lll}(0.418,0.964) & (0.000,0.518) & (0.000,0.348)\end{array}$

$2 \quad[0.000,0.515] \quad[0.261,0.814] \quad[0.000,0.513]$ $\begin{array}{lll}(0.000,0.528) & (0.244,0.830) & (0.000,0.525)\end{array}$

$3[0.000,0.336] \quad[0.000,0.462] \quad[0.467,0.979]$ $\begin{array}{lll}(0.000,0.346) & (0.000,0.477) \quad(0.452,0.993)\end{array}$
1

$1 \quad[0.517,0.872] \quad[0.051,0.419] \quad[0.000,0.252]$ $\begin{array}{lll}(0.509,0.882) & (0.042,0.427) & (0.000,0.257)\end{array}$

$2[0.026,0.406] \quad[0.355,0.728] \quad[0.049,0.428]$ $\begin{array}{llll}(0.017,0.416) & (0.344,0.738) & (0.041,0.437)\end{array}$

$3[0.000,0.285] \quad[0.042,0.389] \quad[0.515,0.861]$ $\begin{array}{lll}(0.000,0.292) & (0.034,0.399) & (0.504,0.869)\end{array}$
1

$1 \quad[0.422,0.975][0.000,0.512] \quad[0.000,0.347]$ $\begin{array}{lll}(0.414,0.985) & (0.000,0.520) & (0.000,0.352)\end{array}$

$2 \quad[0.000,0.507] \quad[0.256,0.829] \quad[0.000,0.508]$ $\begin{array}{llll}(0.000,0.517) & (0.244,0.840) & (0.000,0.517)\end{array}$

$3[0.000,0.379] \quad[0.000,0.483] \quad[0.421,0.951]$ $\begin{array}{lll}(0.000,0.386) & (0.000,0.494) \quad(0.410,0.960)\end{array}$
1

$10.445,0.900]$ $(0.432,0.916) \quad(0.021,0.502) \quad(0.000,0.312)$

$2 \quad[0.000,0.464] \quad[0.288,0.830] \quad[0.000,0.527]$ $\begin{array}{lll}(0.000,0.478) & (0.272,0.842) & (0.000,0.538)\end{array}$

$3 \quad[0.000,0.359] \quad[0.000,0.468] \quad[0.427,0.936]$ $\begin{array}{llll}(0.000,0.367) & (0.000,0.478) \quad(0.414,0.944)\end{array}$

B. Uniform, Independent Misclassification $(\mathrm{Q}=0.20)$

$$
1
$$

$1 \quad[0.527,0.852] \quad[0.075,0.411] \quad[0.000,0.247]$ $\begin{array}{llll}(0.510,0.868) & (0.060,0.426) & (0.000,0.256)\end{array}$

$2 \quad[0.050,0.416] \quad[0.359,0.712] \quad[0.036,0.421]$ $\begin{array}{llll}(0.035,0.428) & (0.343,0.728) & (0.028,0.432)\end{array}$

$3 \quad[0.000,0.247][0.025,0.374] \quad[0.555,0.868]$ $\begin{array}{lll}(0.000,0.257) & (0.013,0.388) & (0.541,0.885)\end{array}$
$[0.530,0.817] \quad[0.120,0.405] \quad[0.000,0.221]$ $\begin{array}{lll}(0.519,0.832) & (0.106,0.416) & (0.000,0.228)\end{array}$

$2 \quad[0.037,0.369] \quad[0.384,0.727] \quad[0.088,0.430]$ $\begin{array}{lll}(0.026,0.382) & (0.368,0.738) & (0.069,0.441)\end{array}$

$3 \quad[0.000,0.274] \quad[0.050,0.384] \quad[0.531,0.850]$ $(0.000,0.282) \quad(0.042,0.393) \quad(0.516,0.858)$

$(0.000,0.292) \quad(0.034,0.399) \quad(0.504,0.869)$

Notes: Outcome = OECD equivalized income. Point estimates for bounds provided in brackets obtained using 100 subsamples of size N/2 for bias correction. 90\% Imbens-Manski confidence intervals for the bounds provided in parentheses obtained using 250 subsamples of size N/2. See Table 3 and text for further details. 
Table 7. Tercile Transition Matrices: Monotonicity + Level Set Restrictions.

I. No Shape Restrictions

A. Arbitrary, Independent Misclassification $(Q=0.20)$

$$
1
$$
3

$1 \quad[0.435,0.919] \quad[0.053,0.503] \quad[0.000,0.281]$ $\begin{array}{lll}(0.418,0.936) & (0.034,0.518) & (0.000,0.308)\end{array}$

$2 \quad[0.000,0.405] \quad[0.270,0.805] \quad[0.008,0.360]$ $\begin{array}{lll}(0.000,0.424) & (0.254,0.820) & (0.000,0.381)\end{array}$

$\begin{array}{lllll}3 & {[0.000,0.336]} & {[0.006,0.462]} & {[0.467,0.928]}\end{array}$ $\begin{array}{lll}(0.000,0.346) & (0.000,0.477) \quad(0.452,0.949)\end{array}$

B. Uniform, Independent Misclassification $(Q=0.20)$
$1 \quad[0.527,0.842] \quad[0.113,0.411] \quad[0.000,0.152]$ $\begin{array}{lll}(0.510,0.858) & (0.096,0.426) & (0.000,0.187)\end{array}$

$2 \quad[0.065,0.346] \quad[0.368,0.705] \quad[0.074,0.306]$ $\begin{array}{llll}(0.050,0.364) & (0.352,0.720) & (0.062,0.326)\end{array}$

$3 \quad[0.000,0.241] \quad[0.064,0.374] \quad[0.555,0.839]$ $(0.000,0.257) \quad(0.046,0.388) \quad(0.541,0.864)$
1

$[0.422,0.937] \quad[0.018,0.512] \quad[0.000,0.317]$ $\begin{array}{lll}(0.414,0.951) & (0.006,0.520) & (0.000,0.327)\end{array}$

$2 \quad[0.000,0.393] \quad[0.262,0.818] \quad[0.001,0.403]$ $\begin{array}{lll}(0.000,0.405) & (0.251,0.829) \quad(0.000,0.417)\end{array}$

$3[0.000,0.344] \quad[0.010,0.483] \quad[0.421,0.922]$

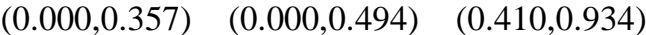

II. With Shape Restrictions

A. Arbitrary, Independent Misclassification $(\mathrm{Q}=0.20)$$$
2
$$

3

$1 \quad[0.435,0.919] \quad[0.053,0.503] \quad[0.000,0.281]$ $\begin{array}{llll}(0.418,0.936) & (0.034,0.518) & (0.000,0.308)\end{array}$

$2 \quad[0.000,0.405] \quad[0.270,0.805] \quad[0.008,0.360]$ $\begin{array}{llll}(0.000,0.424) & (0.254,0.820) & (0.000,0.381)\end{array}$

$3 \quad[0.000,0.336] \quad[0.006,0.462] \quad[0.467,0.928]$ $\begin{array}{lll}(0.000,0.346) & (0.000,0.477) \quad(0.452,0.949)\end{array}$
1

$1 \quad[0.517,0.869] \quad[0.083,0.419] \quad[0.000,0.195]$ $\begin{array}{lll}(0.509,0.882) & (0.071,0.427) & (0.000,0.213)\end{array}$

$2[0.050,0.336] \quad[0.361,0.715] \quad[0.073,0.342]$ $\begin{array}{llll}(0.042,0.347) & (0.350,0.726) & (0.064,0.355)\end{array}$

$3[0.000,0.222] \quad[0.070,0.389] \quad[0.515,0.834]$ $\begin{array}{lll}(0.000,0.239) & (0.059,0.399) & (0.504,0.848)\end{array}$
1

$1 \quad[0.422,0.937][0.018,0.512] \quad[0.000,0.317]$ $\begin{array}{llll}(0.414,0.951) & (0.006,0.520) & (0.000,0.327)\end{array}$

$2 \quad[0.000,0.393] \quad[0.262,0.818] \quad[0.001,0.402]$ $\begin{array}{llll}(0.000,0.405) & (0.251,0.829) & (0.000,0.414)\end{array}$

$3[0.000,0.344][0.010,0.483] \quad[0.421,0.922]$ $\begin{array}{lll}(0.000,0.357) & (0.000,0.494) & (0.410,0.934)\end{array}$
1

$1 \quad[0.445,0.893]$ $(0.432,0.909)$

$2[0.004,0.382]$ $(0.000,0.395)$

$3[0.000,0.329]$ $(0.000,0.345)$

\section{2}

$[0.073,0.489] \quad[0.000,0.264]$ $(0.055,0.502) \quad(0.000,0.290)$ $[0.299,0.791] \quad[0.039,0.374]$ $(0.285,0.804) \quad(0.025,0.393)$ $[0.006,0.468] \quad[0.427,0.927]$ $(0.000,0.478) \quad(0.414,0.944)$

B. Uniform, Independent Misclassification $(\mathrm{Q}=0.20)$

$\begin{array}{ccccc} & \mathbf{1} & \mathbf{2} & \mathbf{3} \\ \mathbf{1} & {[0.527,0.842]} & {[0.113,0.411]} & {[0.000,0.152]}\end{array}$ $\begin{array}{lll}(0.510,0.858) & (0.096,0.426) & (0.000,0.187)\end{array}$

$2 \quad[0.065,0.346] \quad[0.368,0.705] \quad[0.074,0.306]$ $\begin{array}{lll}(0.050,0.364) & (0.352,0.720) & (0.062,0.326)\end{array}$

$3[0.000,0.241][0.064,0.374] \quad[0.555,0.839]$ $\begin{array}{lll}(0.000,0.257) & (0.046,0.388) & (0.541,0.864)\end{array}$
1

$1[0.530,0.817]$ $(0.519,0.832)$

$2[0.071,0.327]$ $(0.060,0.338)$

$3[0.000,0.201]$ $(0.000,0.217)$

\section{2}

$[0.123,0.405]$ $(0.107,0.416)$ [0.394,0.695] $(0.381,0.707)$ $[0.078,0.384]$ $(0.067,0.393)$

\section{3}

$[0.000,0.130]$ $(0.000,0.164)$ $[0.102,0.312]$ $(0.088,0.332)$ $[0.531,0.820]$ $(0.516,0.845)$ $\begin{array}{llll}(0.000,0.239) & (0.059,0.399) & (0.504,0.848)\end{array}$

$1[0.517,0.869] \quad[0.083,0.419] \quad[0.000,0.195]$ $\begin{array}{llll}(0.509,0.882) & (0.071,0.427) & (0.000,0.213)\end{array}$

$2 \quad[0.050,0.336] \quad[0.361,0.715] \quad[0.073,0.342]$ $\begin{array}{llll}(0.042,0.347) & (0.350,0.726) & (0.064,0.355)\end{array}$

$3 \quad[0.000,0.222] \quad[0.070,0.389] \quad[0.515,0.834]$ $\begin{array}{lcccc} & \mathbf{1} & \mathbf{2} & \mathbf{3} \\ 1 & {[0.445,0.893]} & {[0.073,0.489]} & {[0.000,0.264]}\end{array}$ $\begin{array}{lll}(0.432,0.909) & (0.055,0.502) & (0.000,0.290)\end{array}$

$2 \quad[0.004,0.382] \quad[0.299,0.791] \quad[0.039,0.374]$ $\begin{array}{llll}(0.000,0.395) & (0.285,0.804) & (0.025,0.393)\end{array}$

$3 \quad[0.000,0.329] \quad[0.006,0.468] \quad[0.427,0.927]$ $\begin{array}{lll}(0.000,0.345) & (0.000,0.478) & (0.414,0.944)\end{array}$

Notes: Outcome = OECD equivalized income. Point estimates for bounds provided in brackets obtained using 100 subsamples of size N/2 for bias correction. 90\% Imbens-Manski confidence intervals for the bounds provided in parentheses obtained using 250 subsamples of size N/2. See Table 4 and text for further details. 
Table 8. Tercile Transition Matrices: Summary Mobility Measures.

2004-2008 Panel

I. Expected Exit Time: Q1
CPME
AM
$(3.037,3.278)$
AM
LSR + Shape + AIM
LSR + Shape + UIM
$\mathrm{M}+\mathrm{LSR}+$ Shape + AIM
$\mathrm{M}+\mathrm{LSR}+$ Shape + UIM
$(1.596,125.971)$
$(1.899,9.279)$
$(1.724,27.805)$
$(2.048,7.641)$
$(1.724,15.482)$
$(2.048,6.980)$

II. Expected Exit Time: Q3

$$
\text { CPME }
$$

AM

$\mathrm{UM}$

LSR + Shape + AIM

LSR + Shape + UIM

M + LSR + Shape + AIM

$\mathrm{M}+\mathrm{LSR}+$ Shape + UIM

III. Upward Mobility

$\begin{array}{ll}\text { CPME } & (0.458,0.494) \\ \text { AM } & (0.012,0.940) \\ \text { UM } & (0.162,0.790) \\ \text { LSR + Shape + AIM } & (0.054,0.870) \\ \text { LSR + Shape + UIM } & (0.196,0.732) \\ \text { M + LSR + Shape + AIM } & (0.097,0.870) \\ \text { M + LSR + Shape + UIM } & (0.215,0.732)\end{array}$

IV. Downward Mobility

$\begin{array}{ll}\text { CPME } & (0.446,0.477) \\ \text { AM } & (0.001,0.923) \\ \text { UM } & (0.150,0.773) \\ \text { LSR + Shape + AIM } & (0.007,0.823) \\ \text { LSR + Shape + UIM } & (0.169,0.690) \\ \text { M + LSR + Shape + AIM } & (0.078,0.823) \\ \text { M + LSR + Shape + UIM } & (0.205,0.690)\end{array}$

2008-2012 Panel

I. Expected Exit Time: Q1

CPME

AM

$(3.105,3.257)$

$(1.612,114.694)$

$(1.922,9.198)$

$(1.711,66.741)$

$(2.042,8.469)$

$(1.711,20.295)$

$(2.042,8.402)$

II. Expected Exit Time: Q3

CPME

(3.091,3.260)

AM

$(1.609,116.592)$

$(1.917,9.210)$

$\mathrm{LSR}+$ Shape + AIM

$(1.698,24.599)$

LSR + Shape + UIM

$(2.020,7.615)$

M + LSR + Shape + AIM

(1.698,14.765)

M + LSR + Shape + UIM

$(2.020,6.449)$

III. Upward Mobility

CPME

$(0.461,0.483)$

AM

$(0.013,0.931)$

$\mathrm{UM}$

$(0.163,0.781)$

LSR + Shape + AIM

$(0.022,0.877)$

LSR + Shape + UIM

$(0.177,0.735)$

$\mathrm{M}+\mathrm{LSR}+$ Shape + AIM

$(0.074,0.877)$

M + LSR + Shape + UIM

$(0.179,0.735)$

IV. Downward Mobility

$\begin{array}{ll}\text { CPME } & (0.460,0.485) \\ \text { AM } & (0.013,0.933) \\ \text { UM } & (0.163,0.783) \\ \text { LSR + Shape + AIM } & (0.061,0.883) \\ \text { LSR + Shape + UIM } & (0.197,0.743) \\ \text { M + LSR + Shape + AIM } & (0.102,0.883) \\ \text { M + LSR + Shape + UIM } & (0.233,0.743)\end{array}$

$(0.460,0.485)$

$(0.163,0.783)$

$(0.197,0.743)$

$(0.233,0.743)$

Notes: Outcome $=$ OECD equivalized income. CPME = classification-preserving measurement error. AM = arbitrary misclassification. UM = uniform misclassification. $\mathrm{I}=$ independence. $\mathrm{LSR}=$ level set restrictions. $\mathrm{M}=$ monotonicity. $90 \%$ confidence intervals for bounds provided in parentheses based on estimates in Tables 5-7. See text for further details. 


\title{
Partial Identification of Economic Mobility: With an Application to the United States
}

\author{
Supplemental Appendix
}




\section{A Literature Review}

We identify three general approaches to handling measurement error in the study of mobility in the existing literature: (i) ignore it, (ii) ad hoc data approaches, and (iii) structural approaches. The first, and most common, approach is to note the problem and then ignore it. Pavlopoulos et al. (2012, p. 750) state that "despite the enormous bias that measurement error can cause in the estimation of wage dynamics, most relevant studies ignore this phenomenon." Lee et al. (2017, p. 37) write that "most studies of income and poverty dynamics have ignored potential measurement error biases in the transition matrices, although the presence of measurement error in both income and expenditure survey data has been widely acknowledged."

The second strategy we refer to as ad hoc data approaches. Trimming is one example and refers to the practice of deleting a fraction (say, 1\%) of the poorest and richest observations in the sample. Jäntti and Jenkins (2015, p. 862) note that trimming has been "applied in virtually every study cited." The motivation for trimming is the removal of outliers that may represent measurement error (e.g., Maasoumi and Trede 2001). The drawbacks to trimming include the fact that outliers may arise for reasons other than measurement error and that it does not address measurement error outside the tails of the distribution.

A second example of an ad hoc approach is to average income data over several years. Thus, when computing mobility between two points in time, income in the initial (terminal) period is taken as, say, the three-year average around the true initial (terminal) period. Such a strategy was popularized in Solon (1992); see Bhattacharya and Mazumder (2011) and Bradbury (2016) for more recent examples. The motivation for averaging income over several periods is to smooth away measurement error. However, there are several drawbacks to this procedure. First, averaging smooths away all time-varying idiosyncratic sources of income variation, regardless of whether the variation arises from measurement error or legitimate shocks to income. Second, averaging will not remove measurement error that is persistent over time. Finally, averaging requires data from more than two time periods, a requirement that may be prohibitive.

A third example of an ad hoc approach is the pseudo-panel estimator in Antman and 
McKenzie (2007), although the approach can also be applied with genuine panel data. Here, rather than averaging income over several periods for each observation, income is averaged over individuals assigned to the same cohort within each time period. Measures of mobility are then computed using panel data at the cohort level. As in the preceding case, the motivation for averaging income within cohorts and time periods is to smooth away measurement error. Again, though, there are several drawbacks. First, averaging smooths away all time-varying idiosyncratic sources of income variation. Second, cohorts must remain stable over time, which is not assured when using pseudo-panel data, and cohorts must be large. Finally, the definition of cohorts is arbitrary and shrinks the effective sample size.

The final general strategy used in the extant literature we refer to as structural approaches. Approaches falling under this category represent the forefront of the literature and can be sub-divided into two groups. The first group seeks to estimate a scalar measure of mobility: either the correlation coefficient between (true) log incomes in the initial and terminal periods, denoted by $\rho$, or the elasticity of (true) terminal period income with respect to (true) initial period income, denoted by $\beta$ in the following linear regression model

$$
\ln \left(y_{1 i}^{*}\right)=\alpha+\beta \ln \left(y_{0 i}^{*}\right)+\varepsilon_{i},
$$

where $y_{0 i}^{*}\left(y_{1 i}^{*}\right)$ is income in the true initial (terminal) period for observation $i$. Glewwe (2012) notes that if we define $\beta_{R}$ as the coefficient in the reverse regression, given by

$$
\ln \left(y_{0 i}^{*}\right)=\alpha_{R}+\beta_{R} \ln \left(y_{1 i}^{*}\right)+\eta_{i}
$$

then

$$
\operatorname{plim} \sqrt{\widehat{\beta} \cdot \widehat{\beta}_{R}}=\rho
$$

where $\widehat{\beta}$ and $\widehat{\beta}_{R}$ denote the ordinary least squares (OLS) estimates of the corresponding population parameters.

With measurement error, the researcher observes $y_{0 i}$ and $y_{1 i}$. As such, $\widehat{\beta}$ and $\widehat{\beta}_{R}$ are inconsistent and the square root of their product provides a consistent estimate of the correlation between the logs of observed income, not true income. The solution proffered in the 
literature is to recover consistent estimates of $\beta, \beta_{R}$, and $\rho$ via instrumental variables (IV). There are two drawbacks to this approach. First, obtaining credible instruments is extremely difficult (if not impossible). Antman and McKenzie (2007) and Glewwe (2012) offer detailed

examinations of this issue. Second, the scalar nature of $\rho$ and $\beta$ precludes examination of mobility at different parts of the distribution.

The second group of structural approaches are discussed in the main text.

\section{References}

[1] Antman, F. and D. McKenzie (2007), "Earnings Mobility and Measurement Error: A Pseudo-Panel Approach," Economic Development and Cultural Change, 56, 125-162.

[2] Bhattacharya, D. and B. Mazumder (2011), "A Nonparametric Analysis of Black-White Differences in Intergenerational Income Mobility in the United States," Quantitative Economics, 2, 335-379.

[3] Maasoumi, E. and M. Trede (2001), "Comparing Income Mobility in Germany and the United States Using Generalized Entropy Mobility Measures," Review of Economics and Statistics, 83, 551-559.

[4] Solon, G. (1992), "Intergenerational Income Mobility in the United States," American Economic Review, 82, 393-408. 


\section{B Misclassification Probabilities}

We can write the elements of $P_{0,1}^{*}$ as

$$
\begin{aligned}
& p_{k l}^{*}=\frac{\operatorname{Pr}\left(y_{0}^{*} \in k, y_{1}^{*} \in l\right)}{\operatorname{Pr}\left(y_{0}^{*} \in k\right)}
\end{aligned}
$$

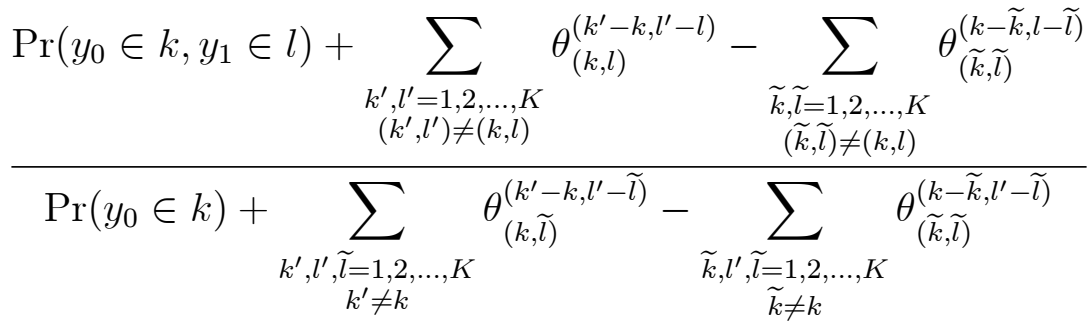

$$
\begin{aligned}
& r_{k l}+\sum_{\substack{k^{\prime}, l^{\prime}=1,2, \ldots, K \\
\left(k^{\prime}, l^{\prime}\right) \neq(k, l)}} \theta_{(k, l)}^{\left(k^{\prime}-k, l^{\prime}-l\right)}-\sum_{\substack{\widetilde{k}, \widetilde{l}=1,2, \ldots, K \\
(\widetilde{h} \widetilde{l}) \neq(k, l)}} \theta_{(\widetilde{k}, \widetilde{l})}^{(k-\widetilde{k}, l-\widetilde{l})}
\end{aligned}
$$



$$
\begin{aligned}
& =K\left[\operatorname{Pr}\left(y_{0} \in k, y_{1} \in l\right)+\sum_{\substack{k^{\prime}, l^{\prime}=1,2, \ldots, K \\
\left(k^{\prime}, l^{\prime}\right) \neq(k, l)}} \theta_{(k, l)}^{\left(k^{\prime}-k, l^{\prime}-l\right)}-\sum_{\substack{\widetilde{k}, \widetilde{l}=1,2, \ldots, K \\
(\widetilde{k}, \widetilde{l}) \neq(k, l)}} \theta_{(\widetilde{k}, \widetilde{l})}^{(k-\widetilde{k}, l-\widetilde{l})}\right],
\end{aligned}
$$

where the final line holds only in the case of equal-sized partitions. The transition probabilities are not identified from the data alone. The data identify $\operatorname{Pr}\left(y_{0} \in k, y_{1} \in l\right)$ and $\operatorname{Pr}\left(y_{0} \in k\right)$, but not the misclassification parameters, $\theta$. In principal, one can compute sharp bounds by searching across the unknown misclassification parameters. There are $K^{2}\left(K^{2}-1\right)$ misclassification parameters in $P_{0,1}^{*}$. However, the following constraints must hold.

(i) $0 \leq \sum_{\substack{\widetilde{k}, \widetilde{l}=1,2, \ldots, K \\(\widetilde{k}, \widetilde{l}) \neq(k, l)}} \theta_{(\widetilde{k}, \widetilde{l})}^{(k-\widetilde{k}, l-\widetilde{l})} \leq r_{k l}, \quad k, l=1, \ldots, K$

(ii) $0 \leq \sum_{\substack{\widetilde{k}, l^{\prime}, \widetilde{l}=1,2, \ldots, K \\ \widetilde{k} \neq k}} \theta_{(\widetilde{k}, \widetilde{l})}^{\left(k-\widetilde{k}, l^{\prime}-\widetilde{l}\right)} \leq p_{k}, \quad k=1, \ldots, K$

(iii) $0 \leq \sum_{\substack{k^{\prime}, \widetilde{k}, \widetilde{l}=1,2, \ldots, K \\ \widetilde{l} \neq l}} \theta_{(\widetilde{k}, \widetilde{l})}^{\left(k-\widetilde{k}, l^{\prime}-\widetilde{l}\right)} \leq p_{l}, \quad l=1, \ldots, K$ 
The $K^{2}$ inequality constraints in (i) must hold since the fraction of observations incorrectly classified as belonging to partition $(k, l)$ cannot exceed the fraction of observations classified as belonging to this partition. The $K$ inequality constraints in (ii) and (iii) must hold since the fraction of observations incorrectly classified as belonging to partition $k$ in period 0 or partition $l$ in period 1 cannot exceed the fraction of observations classified as belonging to these partitions.

In addition, the following constraints must hold in the case of equal-sized partitions:

$$
\begin{aligned}
& \text { (iv.a) } \sum_{\substack{k^{\prime}, l^{\prime}, \widetilde{l}=1,2, \ldots, K \\
k^{\prime} \neq k}} \theta_{(k, \widetilde{l})}^{\left(k^{\prime}-k, l^{\prime}-\widetilde{l}\right)}-\sum_{\substack{\widetilde{k}, l^{\prime}, \widetilde{l}=1,2, \ldots, K \\
\widetilde{k} \neq k}} \theta_{(\widetilde{k}, \widetilde{l})}^{\left(k-\widetilde{k}, l^{\prime}-\widetilde{l}\right)}=0, \quad k=1, \ldots, K \\
& \text { (v.a) } \sum_{\substack{k^{\prime}, \widetilde{k}, l^{\prime}=1,2, \ldots, K \\
l^{\prime} \neq l}} \theta_{(\widetilde{k}, l)}^{\left(k^{\prime}-\widetilde{k}, l^{\prime}-l\right)}-\sum_{\substack{k^{\prime}, \widetilde{k}, \widetilde{l}=1,2, \ldots, K \\
\vec{l} \neq l}} \theta_{(\widetilde{k}, \widetilde{l})}^{\left(k^{\prime}-\widetilde{k}, l-\widetilde{l}\right)}=0, \quad l=1, \ldots, K \\
& \text { (vi.a) }-r_{k l} \leq \sum_{\substack{k^{\prime}, l^{\prime}=1,2, \ldots, K \\
\left(k^{\prime}, l^{\prime}\right) \neq(k, l)}} \theta_{(k, l)}^{\left(k^{\prime}-k, l^{\prime}-l\right)}-\sum_{\substack{\widetilde{k}, \widetilde{l}=1,2, \ldots, K \\
(\widetilde{k}, \widetilde{l}) \neq(k, l)}} \theta_{(\widetilde{k}, \widetilde{l})}^{(k-\widetilde{k}, l-\widetilde{l})} \leq \frac{1}{K}-r_{k l}, \quad k, l=1, \ldots, K
\end{aligned}
$$

The constraints in (iv.a) and (v.a) follow from the fact that $\operatorname{Pr}\left(y_{0} \in k\right)=\operatorname{Pr}\left(y_{1} \in l\right)=1 / K$. The constraints in (vi.a) follow from the fact that $r_{k l}^{*} \equiv \operatorname{Pr}\left(y_{0}^{*} \in k, y_{1}^{*} \in l\right) \in[0,1 / K]$.

If the partitions are of unequal size, then the following constraints must hold:

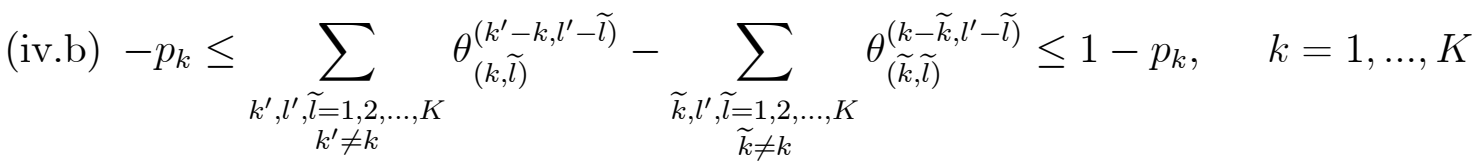

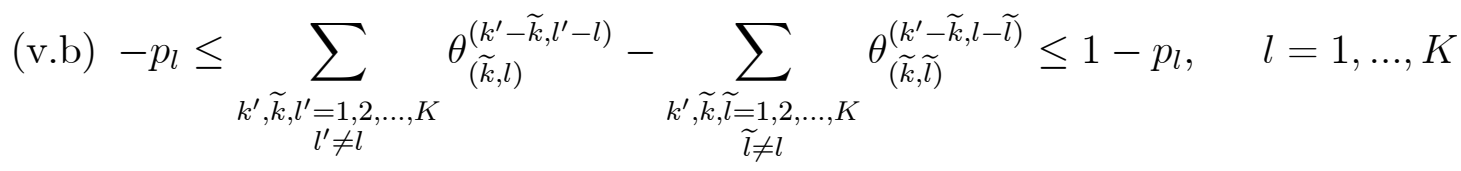

$$
\begin{aligned}
& \text { (vi.b) }-r_{k l} \leq \sum_{\substack{k^{\prime}, l^{\prime}=1,2, \ldots, K \\
\left(k^{\prime}, l^{\prime}\right) \neq(k, l)}} \theta_{(k, l)}^{\left(k^{\prime}-k, l^{\prime}-l\right)}-\sum_{\substack{\widetilde{k}, \widetilde{l}=1,2, \ldots, K \\
(\widetilde{k}, \widetilde{l}) \neq(k, l)}} \theta_{(\widetilde{k}, \widetilde{l})}^{(k-\widetilde{k}, l-\widetilde{l})} \leq 1-r_{k l}, \quad k, l=1, \ldots, K
\end{aligned}
$$

The constraints in (iv.b) and (v.b) follow from the fact that $p_{k}^{*} \equiv \operatorname{Pr}\left(y_{0}^{*} \in k\right) \in[0,1]$ and $p_{l}^{*} \equiv \operatorname{Pr}\left(y_{1}^{*} \in l\right) \in[0,1]$. Finally, the constraints in (vi.b) follow from the fact that $r_{k l}^{*} \equiv \operatorname{Pr}\left(y_{0}^{*} \in k, y_{1}^{*} \in l\right) \in[0,1]$. 


\section{Proofs of Propositions}

Proposition 1. Under Assumption 1, the transition probabilities are nonparametrically identified by

$$
\begin{aligned}
p_{k l}^{*} & =\frac{\operatorname{Pr}\left(y_{0} \in k, y_{1} \in l\right)}{\operatorname{Pr}\left(y_{0} \in k\right)} \\
& =\frac{\mathrm{E}\left[\mathrm{I}\left(y_{0} \in k, y_{1} \in l\right)\right]}{\mathrm{E}\left[\mathrm{I}\left(y_{0} \in k\right)\right]},
\end{aligned}
$$

where $\mathrm{E}[\cdot]$ is the expectation operator and $\mathrm{I}(\cdot)$ is the indicator function.

Proof Assumption 1 implies $Q_{1, k l}=Q_{2, k l}=Q_{3, k}=Q_{4, k}=0, \forall k, l$. Therefore,

$$
\begin{aligned}
p_{k l}^{*} & =\frac{\operatorname{Pr}\left(y_{0} \in k, y_{1} \in l\right)+Q_{1, k l}-Q_{2, k l}}{\operatorname{Pr}\left(y_{0} \in k\right)+Q_{3, k}-Q_{4, k}} \\
& =\frac{\operatorname{Pr}\left(y_{0} \in k, y_{1} \in l\right)}{\operatorname{Pr}\left(y_{0} \in k\right)} \\
& =\frac{\mathrm{E}\left[\mathrm{I}\left(y_{0} \in k, y_{1} \in l\right)\right]}{\mathrm{E}\left[\mathrm{I}\left(y_{0} \in k\right)\right]} \\
& =K \cdot \mathrm{E}\left[\mathrm{I}\left(y_{0} \in k, y_{1} \in l\right)\right],
\end{aligned}
$$

where the last line holds in the case of equal-sized partitions. The sample analog of $p_{k l}^{*}$, denoted by $\widehat{p}_{k l}$, is given by

$$
\begin{aligned}
\widehat{p}_{k l} & =\frac{\frac{1}{N} \sum_{i} \mathrm{I}\left(y_{0 i} \in k, y_{1 i} \in l\right)}{\frac{1}{N} \sum_{i} \mathrm{I}\left(y_{0 i} \in k\right)} \\
& =\frac{\sum_{i} \mathrm{I}\left(y_{0 i} \in k, y_{1 i} \in l\right)}{N / K} \\
& =\frac{K}{N} \sum_{i} \mathrm{I}\left(y_{0 i} \in k, y_{1 i} \in l\right),
\end{aligned}
$$

where the last two lines hold in the case of equal-sized partitions.

Q.E.D.

Proposition 2. Consider a transition matrix, $P_{0,1}^{*}$, with equal-sized partitions. The transi- 
tion probabilities are bounded sharply by

$$
\begin{aligned}
p_{k l}^{*} \in & {\left[\max \left\{K\left(r_{k l}-\widetilde{Q}\right), 1-\sum_{\substack{l^{\prime}=1,2, \ldots, K \\
l^{\prime} \neq l}} U B_{k l^{\prime}}, 1-\sum_{\substack{k^{\prime}=1,2, \ldots, K \\
k^{\prime} \neq k}} U B_{k^{\prime} l}, 0\right\},\right.} \\
& \left.\min \left\{K\left(r_{k l}+\widetilde{Q}\right), 1-\sum_{\substack{l^{\prime}=1,2, \ldots, K \\
l^{\prime} \neq l}} L B_{k l^{\prime}}, 1-\sum_{\substack{k^{\prime}=1,2, \ldots, K \\
k^{\prime} \neq k}} L B_{k^{\prime} l}, 1\right\}\right]
\end{aligned}
$$

where $L B_{k l} \equiv \max \left\{K\left(r_{k l}-\widetilde{Q}\right), 0\right\}, U B_{k l} \equiv \min \left\{K\left(r_{k l}+\widetilde{Q}\right), 1\right\}$, and $\widetilde{Q}=Q / 2$ under Assumption 2(i) and $\widetilde{Q}=Q / K$ under Assumption $2(i i)$.

Proof Proof under Assumption 2(ii) follows from Kreider and Pepper (2008, p. 335) and Horowitz and Manski (1995, Corollary 1.2), with the additional use of the fact that $P_{0,1}^{*}$ is bistochastic (i.e., each row and column must sum to one). The bistochastic property implies that the lower bound on a given element must be at least as great as one minus the sum of the upper bounds for all other elements in the same row or column. Similarly, the property implies that the upper bound on a given element must be no greater than one minus the sum of the lower bounds for all other elements in the same row or column. Assumption 2(i) is identical with the exception that the bidirectionality of misclassification requires offsetting misclassification and thus the maximum value of any $\theta$ is $Q / 2$, not $Q$. No such adjustment is necessary under Assumption 2(ii) since the maximum value of any $\theta$ is already ensured to be less than or equal to $Q / 2$.

To explore the bounds under Assumption 2(i) in more detail, consider a $2 \times 2$ transition matrix and focus on $k=l=1$. Following equation (5) in the main text, $p_{11}^{*}$ is given by

$$
\begin{aligned}
p_{11}^{*} & =\frac{r_{11}+Q_{1,11}-Q_{2,11}}{p_{1}+Q_{3,1}-Q_{4,1}} \\
& =\frac{r_{11}+\left(\theta_{11}^{10}+\theta_{11}^{01}+\theta_{11}^{11}\right)-\left(\theta_{12}^{0-1}+\theta_{21}^{-10}+\theta_{22}^{-1-1}\right)}{p_{1}+\left(\theta_{11}^{10}+\theta_{11}^{11}+\theta_{12}^{10}+\theta_{12}^{1-1}\right)-\left(\theta_{21}^{-10}+\theta_{21}^{-11}+\theta_{22}^{-10}+\theta_{22}^{-1-1}\right)} .
\end{aligned}
$$

To obtain the lower bound, $Q_{2,11}$ and $Q_{3,1}$ must be maximized, while $Q_{1,11}$ and $Q_{4,1}$ must 
be minimized. However, $p_{1}=p_{1}^{*}=1 / K$ with equal-sized partitions, implying

$$
\left(\theta_{11}^{10}+\theta_{11}^{11}+\theta_{12}^{10}+\theta_{12}^{1-1}\right)-\left(\theta_{21}^{-10}+\theta_{21}^{-11}+\theta_{22}^{-10}+\theta_{22}^{-1-1}\right)=0 .
$$

Since $Q_{3,1}$ must equal $Q_{4,1}$, the lower bound is obtained by maximizing $Q_{2,11}$ and setting $Q_{1,11}=Q_{3,1}=Q_{4,1}=0$. As $Q_{2,11}$ has one unique element, $\theta_{12}^{0-1}$, this is feasible.

To proceed, we must determine the largest feasible value of $\theta_{12}^{0-1}$. While $1-\sum_{k, l} \theta_{k l}^{00} \leq Q$, the maximum value of $\theta_{12}^{0-1}$ is $Q / 2$ under Assumption 2(i). To see this, suppose $\theta_{12}^{0-1}=\widehat{Q}>0$ so that $\widehat{Q}$ are misclassified from partition 12 to 11 . It follows then that $\widehat{Q}$ must also be misclassified from some combination of partition 11 into 12 and/or 21 into 22. Thus, it must hold that

$$
\theta_{11}^{01}+\theta_{21}^{01}=\widehat{Q}
$$

Since $\theta_{11}^{01}$ appears in $Q_{1,11}$ and $Q_{1,11}=0$ at the lower bound, it follows that $\theta_{21}^{01}=\widehat{Q}$. This implies

$$
\theta_{12}^{0-1}+\theta_{21}^{01}=2 \widehat{Q}=Q
$$

and thus $\widehat{Q}=Q / 2$.

To obtain the upper bound, $Q_{1,11}$ and $Q_{4,1}$ must be maximized, while $Q_{2,11}$ and $Q_{3,1}$ must be minimized. Again, $p_{1}=p_{1}^{*}=1 / K$ with equal-sized partitions, implying

$$
\left(\theta_{11}^{10}+\theta_{11}^{11}+\theta_{12}^{10}+\theta_{12}^{1-1}\right)-\left(\theta_{21}^{-10}+\theta_{21}^{-11}+\theta_{22}^{-10}+\theta_{22}^{-1-1}\right)=0 .
$$

Since $Q_{3,1}$ must equal $Q_{4,1}$, the upper bound is obtained by maximizing $Q_{1,11}$ and setting $Q_{2,11}=Q_{3,1}=Q_{4,1}=0$. As $Q_{1,11}$ has one unique element, $\theta_{11}^{01}$, this is feasible.

To proceed, we must determine the largest feasible value of $\theta_{11}^{01}$. Again, the maximum value of $\theta_{11}^{01}$ is $Q / 2$ under Assumption 2(i). To see this, suppose $\theta_{11}^{01}=\widehat{Q}>0$ so that $\widehat{Q}$ are misclassified from partition 11 to 12 . It follows then that $\widehat{Q}$ must also be misclassified from some combination of partition 12 into 11 and/or 22 into 21 . Thus, it must hold that

$$
\theta_{12}^{0-1}+\theta_{22}^{0-1}=\widehat{Q}
$$


Since $\theta_{12}^{0-1}$ appears in $Q_{2,11}$ and $Q_{2,11}=0$ at the lower bound, it follows that $\theta_{22}^{0-1}=\widehat{Q}$. This implies

$$
\theta_{11}^{01}+\theta_{22}^{0-1}=2 \widehat{Q}=Q
$$

and thus $\widehat{Q}=Q / 2$.

This argument holds for all $p_{k l}^{*}$ in any $K \times K$ equal-sized transition matrix under Assumption 2(i).

The proof of sharpness follows from Kreider and Pepper (2008, p. 335) and Horowitz and Manski (1995, Corollary 1.2) with the maximum value of $\theta, Q$, replaced by $Q / 2$.

Q.E.D.

Proposition 3. Consider a transition matrix, $P_{0,1}^{*}$, with unequal-sized partitions. Under Assumption 2(i), the transition probabilities are bounded sharply by

$$
p_{k l}^{*} \in\left[\max \left\{\frac{r_{k l}-Q}{p_{k}}, 1-\sum_{\substack{l^{\prime}=1,2, \ldots, K \\ l^{\prime} \neq l}} U B_{k l^{\prime}}, 0\right\}, \min \left\{\frac{r_{k l}+Q}{p_{k}}, 1-\sum_{\substack{l^{\prime}=1,2, \ldots, K \\ l^{\prime} \neq l}} L B_{k l^{\prime}}, 1\right\}\right]
$$

where $L B_{k l} \equiv\left(r_{k l}-Q\right) / p_{k}$ and $U B_{k l} \equiv\left(r_{k l}+Q\right) / p_{k}$. Under Assumption 2(ii), the transition probabilities are bounded sharply by

$$
\begin{aligned}
p_{k l}^{*} \in & {\left[\max \left\{\frac{r_{k l}-Q / K}{p_{k}}, 1-\sum_{\substack{l^{\prime}=1,2, \ldots, K \\
l^{\prime} \neq l}} U B_{k l^{\prime}}, 0\right\},\right.} \\
& \left.\min \left\{\frac{r_{k l}+Q / K}{p_{k}-\min \left\{Q / K, p_{k}\right\}}, 1-\sum_{\substack{l^{\prime}=1,2, \ldots, K \\
l^{\prime} \neq l}} L B_{k l^{\prime}}, 1\right\}\right],
\end{aligned}
$$

where $L B_{k l} \equiv \max \left\{\left(r_{k l}-Q / K\right) / p_{k}\right\}$ and $U B_{k l} \equiv \min \left\{\left(r_{k l}+Q / K\right) /\left(p_{k}-\min \left\{Q / K, p_{k}\right\}\right), 1\right\}$. Proof: See Appendix $C$.

Proof We are interested in bounding $p_{k l}^{*}$, which, following equation (5) in the main text, is given by

$$
p_{k l}^{*}=\frac{r_{k l}+Q_{1, k l}-Q_{2, k l}}{p_{k}+Q_{3, k}-Q_{4, k}} .
$$


$Q_{1, k l}$ and $Q_{4, k}$ share no elements in common; however, they do contain elements corresponding to misclassification in entirely different rows and columns. As such, $Q_{1, k l}+Q_{4, k} \leq Q$ under Assumptions 2(i) and 2(ii). Moreover, under Assumption 2(i) $Q_{1, k l}, Q_{4, k} \leq Q$, while under Assumption 2(ii) $Q_{1, k l}, Q_{4, k} \leq Q / K$. Thus, under Assumption 2(ii), it is feasible that $Q_{1, k l}=Q_{4, k}=Q / K \cdot Q_{2, k l}$ and $Q_{3, k}$ also share no elements in common; however, they only contain elements corresponding to misclassification in the same row or column. As such, $Q_{2, k l}+Q_{3, k} \leq Q$ and $Q_{2, k l}, Q_{3, k} \leq Q$ under Assumptions 2(i). However, $Q_{2, k l}+Q_{3, k} \leq Q / K$ and $Q_{2, k l}, Q_{3, k} \leq Q / K$ under Assumption 2(ii). Thus, under Assumption 2(ii), it is not feasible that $Q_{2, k l}=Q_{3, k}=Q / K$.

Since $r_{k l} \leq p_{k}$, it then follows $p_{k l}^{*}$ must lie in the interval

$$
p_{k l}^{*} \in[0,1] \cap\left[\frac{r_{k l}-Q}{p_{k}}, \frac{r_{k l}+Q}{p_{k}}\right]
$$

under Assumption 2(i), and

$$
p_{k l}^{*} \in[0,1] \cap\left[\frac{r_{k l}-Q / K}{p_{k}}, \frac{r_{k l}+Q / K}{p_{k}-\min \left\{Q / K, p_{k}\right\}}\right]
$$

under Assumption 2(ii). Henceforth, for ease of exposition, we note that if values imply division by zero throughout Appendix $\mathrm{C}$ and we fail to discuss this, in practice one need only reduce the relevant misclassification probability to a marginally lower value. This will produce a large, positive value, allowing the upper limit of one to bind.

The component before the intersection sign follows from the definition of $p_{k l}^{*}$ as a probability. The lower bound in the second component is obtained by setting $Q_{1, k l}=Q_{3, k}=Q_{4, k}=0$ and $Q_{2, k l}=Q$ under Assumption 2(i) or $Q_{2, k l}=Q / K$ under Assumption 2(ii). The upper bound in the second component is obtained by setting $Q_{2, k l}=Q_{3, k}=Q_{4, k}=0$ and $Q_{1, k l}=Q$ under Assumption 2(i) or $Q_{2, k l}=Q_{4, k}=0$ and $Q_{1, k l}=Q_{3, k}=Q / K$ under Assumption 2(ii). Note that subtracting/adding $Q$ to the numerator will always give us a smaller/larger number since $Q$ is a larger percentage of the smaller numerator given $r_{k l} \leq p_{k}$. Equivalently, the 
bounds can be written as

$$
p_{k l}^{*} \in\left[\max \left\{\frac{r_{k l}-Q}{p_{k}}, 0\right\}, \min \left\{\frac{r_{k l}+Q}{p_{k}}, 1\right\}\right]
$$

under Assumption 2(i) and,

$$
p_{k l}^{*} \in\left[\max \left\{\frac{r_{k l}-Q / K}{p_{k}}, 0\right\}, \min \left\{\frac{r_{k l}+Q / K}{p_{k}-\min \{Q / K\}}, 1\right\}\right]
$$

under Assumption 2(ii).

The additional restrictions come from the fact that the transition probabilities in each row must sum to one. Thus, the lower bound of $p_{k l}^{*}$ is bounded by 1 minus the sum of the upper bounds of all other elements in the same row; the upper bound is necessarily bounded by 1 minus the sum of the lower bounds of all other elements in the same row. Formally,

$$
p_{k l}^{*} \geq 1-\sum_{\substack{l^{\prime}=1,2, \ldots, K \\ l^{\prime} \neq l}} U B_{k l^{\prime}}
$$

and

$$
p_{k l}^{*} \leq 1-\sum_{\substack{l^{\prime}=1,2, \ldots, K \\ l^{\prime} \neq l}} L B_{k l^{\prime}}
$$

Next, we show that the bounds in Proposition 3 are sharp. Sharp bounds must exhaust the information about the parameters that is available from the sampling process and the maintained assumptions (Horowitz and Manski 1995). To show the lower bound is sharp is to show that $L B_{k l}=\max \left\{\frac{r_{k l}-Q}{p_{k}}, 0\right\} \leq p_{k l}^{*}$ for all $p_{k l}^{*}$ consistent with Assumption 2 and the data and that there exists a $p_{k l}^{* \prime}$ consistent with Assumption 2 and the data such that

$$
p_{k l}^{* \prime}=\min \left\{\frac{r_{k l}+Q}{p_{k}}, 1-\sum_{\substack{l^{\prime}=1,2, \ldots, K \\ l^{\prime} \neq l}} L B_{k l^{\prime}}, 1\right\}
$$


implying that the endpoint of lower bound may be attained. Define

$$
L B_{k l}= \begin{cases}0 & \text { if } r_{k l} \leq Q \leq 1 \\ \frac{r_{k l}-Q}{p_{k}} & \text { if } 0 \leq Q<r_{k l}\end{cases}
$$

under Assumption 2(i). In this proof, we focus on Assumption 2(i); the proof under Assumption 2(ii) follows a similar argument, but replaces $Q$ with $Q / K$ as well as uses the correct representation of the upper bound.

Case 1: $r_{k l} \leq Q \leq 1$.

Here, $r_{k l}-Q<0$, implying that $L B_{k l}=0$. As such, all $p_{k l}^{*} \geq 0$ are contained in the bounds and thus any $p_{k l}^{*}$ consistent with Assumption 2(i) and the data must be greater than or equal to the lower bound following the property of probabilities. Moreover, since the the values $p_{k l}^{*}$ consistent with Assumption 2(i), the data, and the properties of probabilities include $\left(r_{k l}-Q\right) / p_{k}$, then $p_{k l}^{* \prime}=0$ is a feasible value of $p_{k l}^{*}$ and hence the lower bound may be attained.

Case 2: $0 \leq Q<r_{k l}$.

Following Equation (5) from the main text,

$$
p_{k l}^{*}=\frac{r_{k l}+Q_{1, k l}-Q_{2, k l}}{p_{k}+Q_{3, k}-Q_{4, k}} \geq \frac{r_{k l}-Q_{2, k l}}{p_{k}+Q_{3, k}}
$$

Since $Q_{2, k l}+Q_{3, k} \leq Q$ under Assumption 2(i), the smallest value of $p_{k l}^{*}$ consistent with the maintained assumptions and the data is either $\left(r_{k l}-Q\right) / p_{k}$ or $r_{k l} /\left(p_{k}+Q\right)$. Moreover, since $r_{k l} \leq p_{k}$ due to the fact that an event $E\left\{y_{0} \in k, y_{1} \in l\right\} \subseteq E\left\{y_{0} \in k\right\}$, we have

$$
\frac{r_{k l}-Q}{p_{k}} \leq \frac{r_{k l}}{p_{k}+Q}
$$

as long as $r_{k l}-Q \leq p_{k}$. Note that $r_{k l}-Q \leq p_{k}$ must hold since $p_{k l}^{*} \in[0,1]$ and $p_{k l}^{*} \geq$ $\left(r_{k l}-Q\right) / p_{k}$. Therefore, all values of $p_{k l}^{*}$ consistent with the maintained assumptions and the data are at least as great as the lower bound, and the lower bound is attained at 


$$
p_{k l}^{* \prime}=\left(r_{k l}-Q\right) / p_{k} \text {. If } \frac{r_{k l}-Q}{p_{K}}<1-\sum_{\substack{l^{\prime}=1,2, \ldots, K \\ l^{\prime} \neq l}} U B_{k l^{\prime}},
$$

since the row transition probabilities must sum to 1 , then the lower bound of $p_{k l}^{*}$ is obtained at

$$
p_{k l}^{* \prime}=1-\sum_{\substack{l^{\prime}=1,2, \ldots, K \\ l^{\prime} \neq l}} U B_{k l^{\prime}}
$$

which must be a feasible value given the maintained assumptions and the data if

$$
\frac{r_{k l}-Q}{p_{K}}<1-\sum_{\substack{l^{\prime}=1,2, \ldots, K \\ l^{\prime} \neq l}} U B_{k l^{\prime}}
$$

and $p_{k l}^{* \prime}=\left(r_{k l}-Q\right) / p_{k}$ is feasible. Thus, we have show that the smallest possible value of $p_{k l}^{*}$ that is consistent with Assumption 2(i) and the data is exactly attained at

$$
p_{k l}^{* \prime}=\min \left\{\frac{r_{k l}+Q}{p_{k}}, 1-\sum_{\substack{l^{\prime}=1,2, \ldots, K \\ l^{\prime} \neq l}} U B_{k l^{\prime}}, 1\right\} .
$$

Similarly, to show that $U B_{k l}$ is the sharp upper bound is to show that $U B_{k l}=\max \left\{\frac{r_{k l}+Q}{p_{k}}, 0\right\} \geq$ $p_{k l}^{*}$ for all $p_{k l}^{*}$ consistent with Assumption 2(i) and the data and that there exists a $p_{k l}^{* \prime}$ consistent with Assumption 2 and the data such that

$$
p_{k l}^{* \prime}=\min \left\{\frac{r_{k l}+Q}{p_{k}}, 1-\sum_{\substack{l^{\prime}=1,2, \ldots, K \\ l^{\prime} \neq l}} L B_{k l^{\prime}}, 1\right\}
$$

implying that the endpoint of lower bound may be attained. Define

$$
U B_{k l}= \begin{cases}1 & \text { if } Q \geq p_{k}-r_{k l} \\ \frac{r_{k l}+Q}{p_{k}} & \text { if } 0 \leq Q<p_{k}-r_{k l}\end{cases}
$$

Case 1: $Q \geq p_{k}-r_{k l}$. 
Here, $r_{k l}+Q \geq p_{k}$, implying that $U B_{k l}=1$. As such, all $p_{k l}^{*} \leq 0$ are contained in the bounds and thus any $p_{k l}^{*}$ consistent with Assumption 2(i) and the data must be less than or equal to the upper bound following the property of probabilities. Moreover, since the the values $p_{k l}^{*}$ consistent with Assumption 2(i), the data, and the properties of probabilities include $\left(r_{k l}+Q\right) / p_{k}$, then $p_{k l}^{* \prime}=1$ is a feasible value of $p_{k l}^{*}$ and hence the upper bound may be attained.

Case 2: $0 \leq Q<p_{k}-r_{k l}$.

Again, following Equation (5) from the main text,

$$
p_{k l}^{*}=\frac{r_{k l}+Q_{1, k l}-Q_{2, k l}}{p_{k}+Q_{3, k}-Q_{4, k}} \leq \frac{r_{k l}+Q_{1, k l}}{p_{k}-Q_{4, k}}
$$

Since $Q_{1, k l}+Q_{4, k} \leq Q$ under Assumption 2(i), the largest value of $p_{k l}^{*}$ consistent with the maintained assumptions and the data is either $\left(r_{k l}+Q\right) / p_{k}$ or $r_{k l} /\left(p_{k}-Q\right)$. Moreover, since $r_{k l}+Q \leq p_{k}$, we have

$$
\frac{r_{k l}}{p_{k}-Q} \leq \frac{r_{k l}+Q}{p_{k}}
$$

and $p_{k l}^{*} \leq\left(r_{k l}+Q\right) / p_{k}$. Therefore, all values of $p_{k l}^{*}$ consistent with the maintained assumptions and the data are no greater than the upper bound, and the upper bound is attained at $p_{k l}^{* \prime}=\left(r_{k l}+Q\right) / p_{k}$. If

$$
\frac{r_{k l}+Q}{p_{K}}>1-\sum_{\substack{l^{\prime}=1,2, \ldots, K \\ l^{\prime} \neq l}} L B_{k l^{\prime}}
$$

since the row transition probabilities must sum to 1 , then the upper bound of $p_{k l}^{*}$ is obtained at

$$
p_{k l}^{* \prime}=1-\sum_{\substack{l^{\prime}=1,2, \ldots, K \\ l^{\prime} \neq l}} L B_{k l^{\prime}}
$$

which must be a feasible value given the maintained assumptions and the data if

$$
\frac{r_{k l}+Q}{p_{K}}>1-\sum_{\substack{l^{\prime}=1,2, \ldots, K \\ l^{\prime} \neq l}} L B_{k l^{\prime}}
$$

and $p_{k l}^{* \prime}=\left(r_{k l}+Q\right) / p_{k}$ is feasible. Thus, we have show that the largest possible value of $p_{k l}^{*}$ 
that is consistent with Assumption 2(i) and the data is exactly attained at

$$
p_{k l}^{* \prime}=\min \left\{\frac{r_{k l}+Q}{p_{k}}, 1-\sum_{\substack{l^{\prime}=1,2, \ldots, K \\ l^{\prime} \neq l}} L B_{k l^{\prime}}, 1\right\} .
$$

Q.E.D.

Proposition 4. Under Assumption 3, the four elements of a $2 \times 2$ transition matrix with unequal-sized partitions are bounded sharply by

$$
\begin{aligned}
& p_{11}^{*} \in\left[\max \left\{\frac{r_{11}}{p_{1}+\min \left\{\widetilde{Q}, 1-p_{1}\right\}}, 1-U B_{12}, 0\right\}, \min \left\{\frac{r_{11}+\widetilde{Q}}{p_{1}}, 1-L B_{12}, 1\right\}\right] \\
& p_{12}^{*} \in\left[\max \left\{\frac{r_{12}-\widetilde{Q}}{p_{1}}, 1-U B_{11}, 0\right\}, \min \left\{\frac{r_{12}+\min \left\{\widetilde{Q}, 1-p_{1}\right\}}{p_{1}+\min \left\{\widetilde{Q}, 1-p_{1}\right\}}, 1-L B_{11}, 1\right\}\right] \\
& p_{21}^{*} \in\left[\max \left\{\frac{r_{21}-\min \left\{\widetilde{Q}, p_{2}\right\}}{p_{2}-\min \left\{\widetilde{Q}, p_{2}\right\}}, 1-U B_{22}, 0\right\}, \min \left\{\frac{r_{21}+\widetilde{Q}}{p_{2}-\widetilde{\widetilde{Q}}}, 1-L B_{22}, 1\right\}\right] \\
& p_{22}^{*} \in\left[\max \left\{\frac{r_{22}-\widetilde{Q}}{p_{2}}, 1-U B_{21}, 0\right\}, \min \left\{\frac{r_{22}}{p_{2}-\min \left\{\widetilde{Q}, p_{2}\right\}}, 1-L B_{21}, 1\right\}\right],
\end{aligned}
$$

where $L B_{k l}$ and $U B_{k l}$ denote the lower and upper bounds of $p_{k l}^{*}$, respectively. Under Assumption $2(i), \widetilde{Q}=Q$ and $\widetilde{\widetilde{Q}}=0$. Under Assumption $2(i i), \widetilde{Q}=Q / 2$ and $\widetilde{\widetilde{Q}}=\min \left\{\widetilde{Q}, p_{2}\right\}$. 
Proof Consider a $2 \times 2$ transition matrix with unequal-sized partitions. Following equation (5) in the main text, the elements of the matrix are given by

$$
\begin{aligned}
p_{11}^{*} & =\frac{r_{11}+Q_{1,11}-Q_{2,11}}{p_{1}+Q_{3,1}-Q_{4,1}} \\
& =\frac{r_{11}+\left(\theta_{11}^{10}+\theta_{11}^{01}+\theta_{11}^{11}\right)-\left(\theta_{12}^{0-1}+\theta_{21}^{-10}+\theta_{22}^{-1-1}\right)}{p_{1}+\left(\theta_{11}^{10}+\theta_{11}^{11}+\theta_{12}^{10}+\theta_{12}^{1-1}\right)-\left(\theta_{21}^{-10}+\theta_{21}^{-11}+\theta_{22}^{-10}+\theta_{22}^{-1-1}\right)} \\
p_{12}^{*} & =\frac{r_{12}+Q_{1,12}-Q_{2,12}}{p_{1}+Q_{3,1}-Q_{4,1}} \\
& =\frac{r_{12}+\left(\theta_{12}^{10}+\theta_{12}^{0-1}+\theta_{12}^{1-1}\right)-\left(\theta_{11}^{01}+\theta_{21}^{-11}+\theta_{22}^{-10}\right)}{p_{1}+\left(\theta_{11}^{10}+\theta_{11}^{11}+\theta_{12}^{10}+\theta_{12}^{1-1}\right)-\left(\theta_{21}^{-10}+\theta_{21}^{-11}+\theta_{22}^{-10}+\theta_{22}^{-1-1}\right)} \\
p_{21}^{*} & =\frac{r_{21}+Q_{1,21}-Q_{2,21}}{p_{2}+Q_{3,2}-Q_{4,2}} \\
& =\frac{r_{21}+\left(\theta_{21}^{-10}+\theta_{21}^{01}+\theta_{21}^{-11}\right)-\left(\theta_{11}^{10}+\theta_{12}^{1-1}+\theta_{22}^{0-1}\right)}{p_{2}+\left(\theta_{21}^{-10}+\theta_{22}^{-10}+\theta_{21}^{-11}+\theta_{22}^{-1-1}\right)-\left(\theta_{11}^{10}+\theta_{11}^{11}+\theta_{12}^{10}+\theta_{12}^{1-1}\right)} \\
p_{22}^{*}= & \frac{r_{22}+Q_{1,22}-Q_{2,22}}{p_{2}+Q_{3,2}-Q_{4,2}} \\
= & \frac{r_{22}+\left(\theta_{22}^{-10}+\theta_{22}^{0-1}+\theta_{22}^{-1-1}\right)-\left(\theta_{11}^{11}+\theta_{12}^{10}+\theta_{21}^{01}\right)}{p_{2}+\left(\theta_{21}^{-10}+\theta_{22}^{-10}+\theta_{21}^{-11}+\theta_{22}^{-1-1}\right)-\left(\theta_{11}^{10}+\theta_{11}^{11}+\theta_{12}^{10}+\theta_{12}^{1-1}\right)} .
\end{aligned}
$$

Under Assumption 3, all $\theta$ s with at least one negative superscript are zero as incorrectly being classified into a lower partition is not allowed. Therefore, the transition probabilities simplify to

$$
\begin{aligned}
& p_{11}^{*}=\frac{r_{11}+\left(\theta_{11}^{10}+\theta_{11}^{01}+\theta_{11}^{11}\right)}{p_{1}+\left(\theta_{11}^{10}+\theta_{11}^{11}+\theta_{12}^{10}\right)} \\
& p_{12}^{*}=\frac{r_{12}+\theta_{12}^{10}-\theta_{11}^{01}}{p_{1}+\left(\theta_{11}^{10}+\theta_{11}^{11}+\theta_{12}^{10}\right)} \\
& p_{21}^{*}=\frac{r_{21}+\theta_{21}^{01}-\theta_{11}^{10}}{p_{2}-\left(\theta_{11}^{10}+\theta_{11}^{11}+\theta_{12}^{10}\right)} \\
& p_{22}^{*}=\frac{r_{22}-\left(\theta_{11}^{11}+\theta_{12}^{10}+\theta_{21}^{01}\right)}{p_{2}-\left(\theta_{11}^{10}+\theta_{11}^{11}+\theta_{12}^{10}\right)}
\end{aligned}
$$

For $p_{11}^{*}$, the lower bound is obtained when $\theta_{11}^{10}=\theta_{11}^{01}=\theta_{11}^{11}=0$ and $\theta_{12}^{10}=Q$ or, equivalently, $Q_{1,11}=0$ and $Q_{3,1}=Q$. Moreover, since $p_{1}+Q_{3,1}=p_{1}^{*} \in[0,1]$, we have 
$0 \leq p_{1}+Q_{3,1} \leq 1$. Therefore,

$$
p_{11}^{*} \geq \frac{r_{11}}{p_{1}+\min \left\{Q, 1-p_{1}\right\}}
$$

which is guaranteed to be nonnegative since all individual terms are nonnegative. The upper bound for $p_{11}^{*}$ is obtained when $\theta_{11}^{10}=\theta_{12}^{10}=\theta_{11}^{11}=0$ and $\theta_{11}^{01}=Q$ or, equivalently, $Q_{1,11}=Q$ and $Q_{3,1}=0$. Moreover, since $p_{11}^{*} \in[0,1]$, we have

$$
p_{11}^{*} \leq \min \left\{\frac{r_{11}+Q}{p_{1}}, 1\right\}
$$

For $p_{12}^{*}$, the lower bound is obtained when $\theta_{12}^{10}=\theta_{11}^{10}=\theta_{11}^{11}=0$ and $\theta_{11}^{01}=Q$ or, equivalently, $Q_{1,12}=Q_{3,1}=0$ and $Q_{2,12}=Q$. Moreover, since $p_{12}^{*} \in[0,1]$, we have

$$
p_{12}^{*} \geq \max \left\{\frac{r_{12}-Q}{p_{1}}, 0\right\}
$$

If $p_{1}+Q \leq 1$, then $r_{12}+Q \leq 1$ as well since $r_{12} \leq p_{1}$. In this case, the upper bound for $p_{12}^{*}$ is obtained when $\theta_{11}^{10}=\theta_{11}^{01}=\theta_{11}^{11}=0$ and $\theta_{12}^{10}=Q$ or, equivalently, $Q_{1,12}=Q_{3,1}=Q$ and $Q_{2,12}=0$, so that

$$
p_{12}^{*} \leq \frac{r_{12}+Q}{p_{1}+Q}
$$

If $p_{1}+Q \geq 1$, the upper bound for $p_{12}^{*}$ is obtained when $\theta_{11}^{10}=\theta_{11}^{01}=\theta_{11}^{11}=0$ and $\theta_{12}^{10}=1-p_{1}$ since $p_{1}+Q_{3,1}=p_{1}^{*} \in[0,1]$. Therefore,

$$
p_{12}^{*} \leq \min \left\{\frac{r_{12}+\min \left\{Q, 1-p_{1}\right\}}{p_{1}+\min \left\{Q, 1-p_{1}\right\}}, 1\right\} .
$$

For $p_{21}^{*}$, the lower bound is obtained when $\theta_{21}^{10}=\theta_{12}^{10}=\theta_{11}^{11}=0$ and $\theta_{11}^{10}=Q$ or, equivalently, $Q_{1,21}=Q_{4,2}=Q$ and $Q_{2,21}=0$. This yields the lower bound since $r_{21} \leq p_{2}$. Moreover, since $r_{21}^{*}, p_{2}^{*} \in[0,1]$, we have

$$
p_{21}^{*} \geq \begin{cases}0 & \text { if } p_{2}=r_{21} \text { and } r_{21} \leq Q \\ \frac{r_{21}-\min \left\{r_{21}, Q\right\}}{p_{2}-\min \left\{r_{21}, Q\right\}} & \text { otherwise }\end{cases}
$$

where the two cases are necessary to explicitly handle the possibility that the bound entails 
division by zero. The upper bound is obtained when $\theta_{21}^{01}=Q$ and $\theta_{11}^{10}=\theta_{12}^{10}=\theta_{11}^{11}=0$ or, equivalently, $Q_{1,21}=Q$ and $Q_{2,21}=Q_{3,2}=Q_{4,2}=0$. Moreover, since $p_{21}^{*} \in[0,1]$, we have $\frac{r_{21}+Q}{p_{2}} \leq 1$, yielding

$$
p_{21}^{*} \leq \min \left\{\frac{r_{21}+Q}{p_{2}}, 1\right\}
$$

For $p_{22}^{*}$, the lower bound is obtained when $\theta_{12}^{10}=\theta_{11}^{10}=\theta_{11}^{11}=0$ and $\theta_{21}^{01}=Q$ or, equivalently, $Q_{4,2}=0$ and $Q_{2,22}=Q$. Moreover, since $p_{22}^{*} \in[0,1]$, we have

$$
p_{22}^{*} \geq \max \left\{\frac{r_{22}-Q}{p_{2}}, 0\right\}
$$

The upper bound is obtained when $\theta_{11}^{11}=\theta_{12}^{10}=\theta_{21}^{01}=0$ and $\theta_{11}^{10}=Q$ or, equivalently, $Q_{4,2}=Q$ and $Q_{2,22}=0$. However, since $p_{2}-Q_{4,2}=p_{2}^{*} \in[0,1]$, we have

$$
p_{22}^{*} \leq \min \left\{\frac{r_{22}}{p_{2}-\min \left\{Q, p_{2}\right\}}, 1\right\}
$$

Finally, note that the additional constraints on the bounds arise from the fact that each row must sum to one. For example, for $p_{11}^{*}$, we have $p_{11}^{*} \geq 1-U B_{12}$ and $p_{11}^{*} \leq 1-L B_{12}$.

Now, we show that the bounds in Proposition 4 are sharp. Following the proof of Proposition 3 , we will show that, given $Q, L B_{k l} \leq p_{k l}^{*}, k, l=1,2$, for all $p_{k l}^{*}$ consistent with Assumptions 2 and 3 and the data, and that there exists a $p_{k l}^{* \prime}$ such that $p_{k l}^{* \prime}=L B_{k l}$. To show the upper bounds are sharp, we will show that, given $Q, p_{k l}^{*} \leq U B_{k l}, k, l=1,2$, for all $p_{k l}^{*}$ given Assumptions 2 and 3 and the data, and that there exists a $p_{k l}^{* \prime}$ such that $p_{k l}^{* \prime}=U B_{k l}$.

Begin with the lower bound for $p_{11}^{*}$. Define

$$
L B_{11}= \begin{cases}\max \left\{r_{11}, 1-U B_{12}\right\} & \text { if } Q \geq 1-p_{1} \\ \max \left\{\frac{r_{11}}{p_{1}+Q}, 1-U B_{12}\right\} & \text { if } 0 \leq Q<1-p_{1}\end{cases}
$$

under Assumption 2(i). In this proof, we focus on Assumption 2(i); the proof under Assumption 2(ii) follows a similar argument, but replaces $Q$ with $Q / K$ as well as uses the correct representation of the upper bound for $p_{21}^{*}$.

Case 1: $Q \geq 1-p_{1}$. 
Under Assumption 3, $p_{11}^{*}$ is given by

$$
p_{11}^{*}=\frac{r_{11}+\left(\theta_{11}^{10}+\theta_{11}^{01}+\theta_{11}^{11}\right)}{p_{1}+\left(\theta_{11}^{10}+\theta_{11}^{11}+\theta_{12}^{10}\right)} .
$$

Since $\sum_{k l} \theta_{k l}^{k^{\prime}-k, l^{\prime}-l} \leq Q, k, l=1,2$, we have

$$
p_{11}^{*}=\frac{r_{11}+\left(\theta_{11}^{10}+\theta_{11}^{01}+\theta_{11}^{11}\right)}{p_{1}+\left(\theta_{11}^{10}+\theta_{11}^{11}+\theta_{12}^{10}\right)}=\frac{r_{11}}{p_{1}+\theta_{12}^{10}} \geq \frac{r_{11}}{p_{1}+Q} .
$$

If $p_{1}+Q>1$, then $p_{1}^{*}=p_{1}+Q>1$, which violates the property of probabilities. In this case, $\theta_{12}^{10}$ must be restricted to $1-p_{1}<Q$ and, hence, $p_{1}+\theta_{12}^{10}=1$. Therefore, $p_{11}^{*} \geq r_{11}$ and all values of $p_{11}^{*}$ consistent with the maintained assumptions and the data are at least as great as the lower bound. Furthermore, the lower bound is attained at $p_{11}^{* \prime}=r_{11}$. If $r_{11}<1-U B_{12}$, the lower bound is obtained at $p_{k l}^{* \prime}=1-U B_{12}$ since the row elements of the transition matrix has to sum to 1. $p_{k l}^{* \prime}=1-U B_{12}$ must be consistent with the maintained assumptions and the data since $p_{11}^{* \prime}=r_{11}$ is feasible and $r_{11}<1-U B_{12}$ in this event.

Case 2: $0 \leq Q<1-p_{1}$.

Here, we have

$$
p_{11}^{*}=\frac{r_{11}+\left(\theta_{11}^{10}+\theta_{11}^{01}+\theta_{11}^{11}\right)}{p_{1}+\left(\theta_{11}^{10}+\theta_{11}^{11}+\theta_{12}^{10}\right)}=\frac{r_{11}}{p_{1}+\theta_{12}^{10}} \geq \frac{r_{11}}{p_{1}+Q} .
$$

Therefore, all values of $p_{11}^{*}$ consistent with the maintained assumptions and the data are at least as great as the lower bound, and the lower bound is attained at $p_{11}^{* \prime}=r_{11} /\left(p_{k}+Q\right)$. If $r_{11} /\left(p_{k}+Q\right)<1-U B_{12}$, the lower bound is obtained at $p_{k l}^{* \prime}=1-U B_{12}$ since the row elements of the transition matrix has to sum to $1 . p_{k l}^{* \prime}=1-U B_{12}$ must be consistent with the maintained assumptions and the data since $p_{11}^{* \prime}=r_{11} /\left(p_{k}+Q\right)$ is feasible and $/\left(p_{k}+Q\right)<1-U B_{12}$ in this event.

The proofs for the sharpness of all other lower and upper bounds in Proposition 4 follow similar arguments..

Q.E.D.

Proposition 5. Denote the bounds on $p_{k l}^{*}$ under some combination of Assumptions 2 and 3 
as

$$
p_{k l}^{*} \in\left[L B_{k l}, U B_{k l}\right] .
$$

Adding Assumption 4 implies the following sharp bounds:

$$
\begin{aligned}
& p_{k l}^{*} \in\left[\max \left\{\sup _{l^{\prime}=1, \ldots, K} L B_{k l^{\prime}}, \sup _{k^{\prime}=1, \ldots, K} L B_{k^{\prime} l}\right\}, U B_{k l}\right] \text { if } k=l \\
& p_{k l}^{*} \in\left[\max \left\{\sup _{l^{\prime} \geq l} L B_{k l^{\prime}}, \sup _{k^{\prime} \leq k} L B_{k^{\prime} l}\right\}, \min \left\{\inf _{k \leq l^{\prime} \leq l} U B_{k l^{\prime}}, \inf _{k \leq k^{\prime} \leq l} U B_{k^{\prime} l}\right\}\right] \text { if } k<l \\
& p_{k l}^{*} \in\left[\max \left\{\sup _{l^{\prime} \leq l} L B_{k l^{\prime}}, \sup _{k^{\prime} \geq k} L B_{k^{\prime} l}\right\}, \min \left\{\inf _{l \leq l^{\prime} \leq k} U B_{k l^{\prime}}, \inf _{l \leq k^{\prime} \leq k} U B_{k^{\prime} l}\right\}\right] \text { if } k>l
\end{aligned}
$$

Proof Denote the bounds on $p_{k l}^{*}$ under some combination of Assumptions 2-3 as

$$
p_{k l}^{*} \in\left[L B_{k l}, U B_{k l}\right] .
$$

Under Assumption $4, p_{k l}^{*}$ is weakly decreasing in $|k-l|$. First, consider $p_{k l}^{*}$ when $k=l$. Since $|k-l|=0, p_{k l}^{*}$ must be at least as large as all other elements in the same row or column. Consequently,

$$
p_{k l}^{*} \geq L B_{k l^{\prime}} \forall l^{\prime}=1, \ldots, K \Longleftrightarrow p_{k l}^{*} \geq \sup _{l^{\prime}=1, \ldots, K} L B_{k l^{\prime}}
$$

and

$$
p_{k l}^{*} \geq L B_{k^{\prime} l} \forall k^{\prime}=1, \ldots, K \Longleftrightarrow p_{k l}^{*} \geq \sup _{k^{\prime}=1, \ldots, K} L B_{k^{\prime} l}
$$

Since assumption 4 provides no additional information regarding the upper bound for $p_{k l}^{*}$ when $k=l$, the bounds become

$$
p_{k l}^{*} \in\left[\max \left\{\sup _{l^{\prime}=1, \ldots, K} L B_{k l^{\prime}}, \sup _{k^{\prime}=1, \ldots, K} L B_{k^{\prime} l}\right\}, U B_{k l}\right] \text { if } k=l
$$

Second, consider $p_{k l}^{*}$ when $k<l$. For all $l^{\prime} \geq l$, so that $k<l \leq l^{\prime}$, we have $|k-l| \leq\left|k-l^{\prime}\right|$. Since $p_{k l}^{*}$ is weakly decreasing in $|k-l|$ under Assumption 4, it follows that

$$
p_{k l}^{*} \geq L B_{k l^{\prime}} \forall l^{\prime} \geq l \Longleftrightarrow p_{k l}^{*} \geq \sup _{l^{\prime} \geq l} L B_{k l^{\prime}}
$$

For all $k \leq l^{\prime} \leq l$, we have $\left|k-l^{\prime}\right| \leq|k-l|$. Under Assumption 4, since $p_{k l}^{*}$ is weakly 
decreasing in $|k-l|$, we have

$$
p_{k l}^{*} \leq U B_{k l^{\prime}} \forall k \leq l^{\prime} \leq l \Longleftrightarrow p_{k l}^{*} \leq \inf _{k \leq l^{\prime} \leq l} U B_{k l^{\prime}}
$$

For all $k^{\prime} \leq k$, so that $k^{\prime} \leq k<l$, we have $\left|k^{\prime}-l\right| \geq|k-l|$. Since $p_{k l}^{*}$ is weakly decreasing in $|k-l|$ under Assumption 4, it follows that

$$
p_{k l}^{*} \geq L B_{k^{\prime} l} \forall k^{\prime} \leq k \Longleftrightarrow p_{k l}^{*} \geq \sup _{k^{\prime} \leq k} L B_{k^{\prime} l}
$$

For all $k \leq k^{\prime} \leq l$, we have $\left|k^{\prime}-l\right| \leq|k-l|$. Since $p_{k l}^{*}$ is weakly decreasing in $|k-l|$ under Assumption 4, it follows that

$$
p_{k l}^{*} \leq U B_{k^{\prime} l} \forall k \leq k^{\prime} \leq l \Longleftrightarrow p_{k l}^{*} \leq \inf _{k \leq k^{\prime} \leq l} U B_{k^{\prime} l}
$$

Thus, the bounds are given by

$$
p_{k l}^{*} \in\left[\max \left\{\sup _{l^{\prime} \geq l} L B_{k l^{\prime}}, \sup _{k^{\prime} \leq k} L B_{k^{\prime} l}\right\}, \min \left\{\inf _{k \leq l^{\prime} \leq l} U B_{k l^{\prime}}, \inf _{k \leq k^{\prime} \leq l} U B_{k^{\prime} l}\right\}\right] \text { if } k<l .
$$

Third, consider $p_{k l}^{*}$ when $k>l$. For all $k^{\prime} \geq k$, so that $l<k \leq k^{\prime}$, then $|k-l| \leq\left|k-l^{\prime}\right|$. Since $p_{k l}^{*}$ is weakly decreasing in $|k-l|$ under Assumption 4, it follows that

$$
p_{k l}^{*} \geq L B_{k^{\prime} l} \forall k^{\prime} \geq k \Longleftrightarrow p_{k l}^{*} \geq \sup _{k^{\prime} \geq k} L B_{k^{\prime} l}
$$

For all $l \leq k^{\prime} \leq k$, then $\left|k^{\prime}-l\right| \leq|k-l|$. Since $p_{k l}^{*}$ is weakly decreasing in $|k-l|$ under Assumption 4, it follows that

$$
p_{k l}^{*} \leq U B_{k^{\prime} l} \forall l \leq k^{\prime} \leq k \Longleftrightarrow p_{k l}^{*} \leq \inf _{l \leq k^{\prime} \leq k} U B_{k^{\prime} l}
$$

For all $l^{\prime} \leq l$, so that $l^{\prime} \leq l<k$, then $\left|k-l^{\prime}\right| \leq|k-l|$. Since $p_{k l}^{*}$ is weakly decreasing in 
$|k-l|$ under Assumption 4, it follows that

$$
p_{k l}^{*} \geq L B_{k l^{\prime}} \forall l^{\prime} \leq l \Longleftrightarrow p_{k l}^{*} \geq \sup _{l^{\prime} \leq l} L B_{k l^{\prime}}
$$

For all $l \leq l^{\prime} \leq k$, then $|k-l| \leq\left|k-l^{\prime}\right|$. Since $p_{k l}^{*}$ is weakly decreasing in $|k-l|$ under Assumption 4, it follows that

$$
p_{k l}^{*} \leq U B_{k l^{\prime}} \forall l \leq l^{\prime} \leq k \Longleftrightarrow p_{k l}^{*} \leq \inf _{l \leq l^{\prime} \leq k} U B_{k l^{\prime}}
$$

Thus, the bounds are given by

$$
p_{k l}^{*} \in\left[\max \left\{\sup _{l^{\prime} \leq l} L B_{k l^{\prime}}, \sup _{k^{\prime} \geq k} L B_{k^{\prime} l}\right\}, \min \left\{\inf _{l \leq l^{\prime} \leq k} U B_{k l^{\prime}}, \inf _{l \leq k^{\prime} \leq k} U B_{k^{\prime} l}\right\}\right] \text { if } k>l .
$$

To prove the sharpness of the bounds in Proposition 5, it is sufficient to show that the bounds includes all values consistent with Assumptions 2, 3, and 4 and the data and the existence of $p_{k l}^{* \prime}$ so that the endpoints of the bounds may be attained. To show the bounds in Proposition 5 exhaust the information provided by the assumptions and the data, we use proof by contradiction.

First, if $k=l$, we have

$$
L B_{k l}=\max \left\{\sup _{l^{\prime}=1, \ldots, K} L B_{k l^{\prime}}, \sup _{k^{\prime}=1, \ldots, K} L B_{k^{\prime} l}\right\} .
$$

Suppose there exists a sufficiently small number $\epsilon>0$ such that $L B_{k l}^{\prime}=L B_{k l}-\epsilon$ satisfies Assumptions 2-4. It follows that

$$
\begin{aligned}
L B_{k l}^{\prime} & =\max \left\{\sup _{l^{\prime}=1, \ldots, K} L B_{k l^{\prime}}, \sup _{k^{\prime}=1, \ldots, K} L B_{k^{\prime} l}\right\}-\epsilon \\
& <\max \left\{\sup _{l^{\prime}=1, \ldots, K} L B_{k l^{\prime}}, \sup _{k^{\prime}=1, \ldots, K} L B_{k^{\prime} l}\right\} .
\end{aligned}
$$

However, the inequality implies that $L B_{k l}^{\prime}$ is smaller than at least one off-diagonal element in the same row or column. This contradicts Assumption 4; $L B_{k l}^{\prime}$ must lie outside the bounds. When $k=l$, Assumption 4 adds no new information to the upper bound of $p_{k l}^{*}$; thus, the 
proof of sharpness follows the proofs of Propositions 2, 3, and 4 .

Second, if $k<l$, we have

$$
p_{k l}^{*} \in\left[L B_{k l}, U B_{k l}\right] .
$$

where

$$
\begin{aligned}
L B_{k l} & =\max \left\{\sup _{l^{\prime} \geq l} L B_{k l^{\prime}}, \sup _{k^{\prime} \leq k} L B_{k^{\prime} l}\right\} \\
U B_{k l} & =\min \left\{\inf _{k \leq l^{\prime} \leq l} U B_{k l^{\prime}}, \inf _{k \leq k^{\prime} \leq l} U B_{k^{\prime} l}\right\} .
\end{aligned}
$$

For the lower bound, suppose there exists a sufficiently small number $\epsilon>0$ so that $L B_{k l}^{\prime}=$ $L B_{k l}-\epsilon$ satisfies Assumptions 2-4. It follows that

$$
\begin{aligned}
L B_{k l}^{\prime} & =\max \left\{\sup _{l^{\prime} \geq l} L B_{k l^{\prime}}, \sup _{k^{\prime} \leq k} L B_{k^{\prime} l}\right\}-\epsilon \\
& <\max \left\{\sup _{l^{\prime} \geq l} L B_{k l^{\prime}}, \sup _{k^{\prime} \leq k} L B_{k^{\prime} l}\right\} .
\end{aligned}
$$

However, the inequality implies that $L B_{k l}^{\prime}$ is smaller than at least one element in the same row or column that is further from the diagonal. This contradicts Assumption $4 ; L B_{k l}^{\prime}$ must lie outside the bounds. For the upper bound, suppose there exists a sufficiently small number $\epsilon>0$ so that $U B_{k l}^{\prime}=U B_{k l}+\epsilon$ satisfies Assumptions 2-4. It follows that

$$
\begin{aligned}
U B_{k l}^{\prime} & =\min \left\{\inf _{k \leq l^{\prime} \leq l} U B_{k l^{\prime}}, \inf _{k \leq k^{\prime} \leq l} U B_{k^{\prime} l}\right\}+\epsilon \\
& >\min \left\{\inf _{k \leq l^{\prime} \leq l} U B_{k l^{\prime}}, \inf _{k \leq k^{\prime} \leq l} U B_{k^{\prime} l}\right\} .
\end{aligned}
$$

However, the inequality implies that $U B_{k l}^{\prime}$ is larger than at least one element in the same row or column that is closer to the diagonal. This contradicts Assumption $4 ; U B_{k l}^{\prime}$ must lie outside the bounds.

Third, if $k>l$, we have

$$
p_{k l}^{*} \in\left[L B_{k l}, U B_{k l}\right] .
$$


where

$$
\begin{aligned}
L B_{k l} & =\max \left\{\sup _{l^{\prime} \leq l} L B_{k l^{\prime}}, \sup _{k^{\prime} \geq k} L B_{k^{\prime} l}\right\} \\
U B_{k l} & =\min \left\{\inf _{l \leq l^{\prime} \leq k} U B_{k l^{\prime}}, \inf _{l \leq k^{\prime} \leq k} U B_{k^{\prime} l}\right\} .
\end{aligned}
$$

For the lower bound, suppose there exists a sufficiently small number $\epsilon>0$ so that $L B_{k l}^{\prime}=$ $L B_{k l}-\epsilon$ satisfies Assumptions 2-4. It follows that

$$
\begin{aligned}
L B_{k l}^{\prime} & =\max \left\{\sup _{l^{\prime} \leq l} L B_{k l^{\prime}}, \sup _{k^{\prime} \geq k} L B_{k^{\prime} l}\right\}-\epsilon \\
& <\max \left\{\sup _{l^{\prime} \leq l} L B_{k l^{\prime}}, \sup _{k^{\prime} \geq k} L B_{k^{\prime} l}\right\} .
\end{aligned}
$$

However, the inequality implies that $L B_{k l}^{\prime}$ is smaller than at least one element in the same row or column that is further from the diagonal. This contradicts Assumption $4 ; L B_{k l}^{\prime}$ must lie outside the bounds. For the upper bound, suppose there exists a sufficiently small number $\epsilon>0$ so that $U B_{k l}^{\prime}=U B_{k l}+\epsilon$ satisfies Assumptions 2-4. It follows that

$$
\begin{aligned}
U B_{k l}^{\prime} & =\min \left\{\inf _{l \leq l^{\prime} \leq k} U B_{k l^{\prime}}, \inf _{l \leq k^{\prime} \leq k} U B_{k^{\prime} l}\right\}+\epsilon \\
& >\min \left\{\inf _{l \leq l^{\prime} \leq k} U B_{k l^{\prime}}, \inf _{l \leq k^{\prime} \leq k} U B_{k^{\prime} l}\right\} .
\end{aligned}
$$

However, the inequality implies that $U B_{k l}^{\prime}$ is larger than at least one element in the same row or column that is closer to the diagonal. This contradicts Assumption $4 ; U B_{k l}^{\prime}$ must lie outside the bounds.

Now, we show the existence of $p_{k l}^{* \prime}$ consistent with the maintained assumptions and the data so that the endpoints bounds may be attained. Since we have the following sharp bounds under Assumption 2 and 3,

$$
\begin{aligned}
L B_{k l} & \leq p_{k l}^{*} \leq U B_{k l} \\
L B_{k^{\prime} l} & \leq p_{k^{\prime} l}^{*} \leq U B_{k^{\prime} l} \\
L B_{k l^{\prime}} & \leq p_{k l^{\prime}}^{*} \leq U B_{k l^{\prime}}
\end{aligned}
$$


$L B_{k l}, U B_{k l}, L B_{k^{\prime} l}, U B_{k^{\prime} l}, L B_{k l^{\prime}}$ and $U B_{k l^{\prime}}$ are all obtainable values under Assumption 2 and 3. Then there must exist suprema of the lower bounds and infima of the upper bounds that are feasible given Assumptions 2 and 3 and the data. Adding Assumption 4, we simply bound $p_{k l}^{*}$ with the infima and suprema of feasible upper and lower bounds in the same row and column with the transition probabilities of interest. Therefore, there exists $p_{k l}^{* \prime}$ so that the endpoints bounds may be attained.

Q.E.D.

Corollary 6.1. Consider a transition matrix, $P_{0,1}^{*}$, with equal- or unequal-sized partitions. Under Assumption 2(i), $p_{k l}^{*}(x)$ is bounded sharply by

$p_{k l}^{*}(x) \in\left[\max \left\{\frac{r_{k l}(x)-\ddot{Q}}{p_{k}(x)}, 1-\sum_{\substack{l^{\prime}=1,2, \ldots, K \\ l^{\prime} \neq l}} U B_{k l^{\prime}}(x), 0\right\}, \min \left\{\frac{r_{k l}(x)+\ddot{Q}}{p_{k}(x)}, 1-\sum_{\substack{l^{\prime}=1,2, \ldots, K \\ l^{\prime} \neq l}} L B_{k l^{\prime}}(x), 1\right\}\right]$

where $L B_{k l}(x) \equiv \max \left\{\left(r_{k l}(x)-\ddot{Q}\right) / p_{k}(x)\right\}, U B_{k l} \equiv \min \left\{\left(r_{k l}(x)+\ddot{Q}\right) / p_{k}(x), 1\right\}$,

$$
\ddot{Q}=\left\{\begin{array}{cl}
\widetilde{Q} & \text { under Assumption } 6 \\
\widetilde{Q} / \operatorname{Pr}(X=x) & \text { otherwise }
\end{array}\right.
$$

and

$$
\widetilde{Q}=\left\{\begin{array}{cl}
Q / 2 & \text { for equal-sized partitions } \\
Q & \text { for unequal-sized partitions }
\end{array}\right.
$$

Proof First consider the bounds with equal-sized partitions. With equal-sized partitions, it must be that

$$
p_{k}^{*}=p_{k}+Q_{3, k}-Q_{4, k}=\frac{1}{K}
$$

implying that $Q_{3, k}=Q_{4, k}$. However, within the subsample with $X=x$, this is no longer the case in general. Now,

$$
p_{k}^{*}=\frac{r_{k l}(x)+Q_{1, k l}(x)-Q_{2, k l}(x)}{p_{k}(x)+Q_{3, k}(x)-Q_{4, k}(x)},
$$

where $p_{k}^{*}(x)=p_{k}(x)+Q_{3, k}(x)-Q_{4, k}(x)$ need not equal $1 / K$. Therefore, we must bound

$$
p_{k}^{*}=\frac{r_{k l}(x)+Q_{1, k l}(x)-Q_{2, k l}(x)}{p_{k}(x)+Q_{3, k}(x)-Q_{4, k}(x)} .
$$


With unequal-sized partitions, this turns out to be also be the formula for $p_{k}^{*}$ since it is already the case that the denominator is not in general equal to $1 / K$.

\section{Case 1: Bounds with equal-sized partitions under Assumptions 2(i) and 6.}

Assumption 6 implies

$$
\begin{aligned}
p_{k}^{*} & =\frac{r_{k l}(x)+Q_{1, k l}(x)-Q_{2, k l}(x)}{p_{k}(x)+Q_{3, k}(x)-Q_{4, k}(x)} \\
& =\frac{r_{k l}(x)+Q_{1, k l}-Q_{2, k l}}{p_{k}(x)+Q_{3, k}-Q_{4, k}} \\
& =\frac{r_{k l}(x)+Q_{1, k l}-Q_{2, k l}}{p_{k}(x)} .
\end{aligned}
$$

It then follows from Proposition 2 that

$$
\frac{r_{k l}(x)-Q / 2}{p_{k}(x)} \leq p_{k l}^{*}(x) \leq \frac{r_{k l}(x)+Q / 2}{p_{k}(x)}
$$

In addition, $p_{k l}^{*}(x) \in[0,1]$ since it is a probability and

$$
p_{k l}^{*}(x) \in\left[1-\sum_{\substack{l^{\prime}=1,2, \ldots, K \\ l^{\prime} \neq l}} U B_{k l^{\prime}}(x), 1-\sum_{\substack{l^{\prime}=1,2, \ldots, K \\ l^{\prime} \neq l}} L B_{k l^{\prime}}(x)\right]
$$

since the rows of $P_{0,1}^{*}(x)$ must sum to one.

Case 2: Bounds with equal-sized partitions under Assumption 2(i).

Under Assumption 2(i),

$$
\begin{aligned}
Q_{j, k l} & =\sum_{x} Q_{j, k l}(x) \operatorname{Pr}(X=x) \leq \frac{Q}{2}, j=1,2 \\
Q_{j, k} & =\sum_{x} Q_{j, k}(x) \operatorname{Pr}(X=x) \leq \frac{Q}{2}, j=3,4 .
\end{aligned}
$$

This implies

$$
Q_{1, k l}(x), Q_{2, k l}(x), Q_{3, k}(x), Q_{4, k}(x) \leq \min \left\{\frac{Q}{2 \operatorname{Pr}(X=x)}\right\}
$$


It then follows from Proposition 3 that

$$
\frac{r_{k l}(x)-Q / 2 \operatorname{Pr}(X=x)}{p_{k}(x)} \leq p_{k l}^{*}(x) \leq \frac{r_{k l}(x)+Q / 2 \operatorname{Pr}(X=x)}{p_{k}(x)}
$$

In addition, $p_{k l}^{*}(x) \in[0,1]$ since it is a probability and

$$
p_{k l}^{*}(x) \in\left[1-\sum_{\substack{l^{\prime}=1,2, \ldots, K \\ l^{\prime} \neq l}} U B_{k l^{\prime}}(x), 1-\sum_{\substack{l^{\prime}=1,2, \ldots, K \\ l^{\prime} \neq l}} L B_{k l^{\prime}}(x)\right]
$$

since the rows of $P_{0,1}^{*}(x)$ must sum to one.

Case 3: Bounds with unequal-sized partitions under Assumptions 2(i) and 6.

This is identical to Case 1 , with $Q / 2$ replaced by $Q$, since misclassification no longer must be bi-directional.

\section{Case 4: Bounds with unequal-sized partitions under Assumption 2(i).}

This is identical to Case 2 , with $Q / 2$ replaced by $Q$, since misclassification no longer must be bi-directional.

The proof of sharpness follows from the proof for Proposition 3, replacing the unconditional probabilities with conditional probabilities and utilizing the correct maximum misclassification rate.

Q.E.D.

Corollary 6.2. Consider a transition matrix, $P_{0,1}^{*}$, with equal- or unequal-sized partitions. 
Under Assumption 2(ii), $p_{k l}^{*}(x)$ is bounded sharply by

$$
\begin{aligned}
p_{k l}^{*}(x) \in & {\left[\max \left\{\frac{r_{k l}(x)-\widetilde{Q}}{p_{k}(x)}, 1-\sum_{\substack{l^{\prime}=1,2, \ldots, K \\
l^{\prime} \neq l}} U B_{k l^{\prime}}(x), 0\right\},\right.} \\
& \left.\min \left\{\frac{r_{k l}(x)+\widetilde{Q}}{p_{k}(x)-\min \left\{\widetilde{Q}, p_{k}(x)\right\}}, 1-\sum_{\substack{l^{\prime}=1,2, \ldots, K \\
l^{\prime} \neq l}} L B_{k l^{\prime}}(x), 1\right\}\right]
\end{aligned}
$$

where $L B_{k l}(x) \equiv \max \left\{\left(r_{k l}(x)-\widetilde{Q}\right) / p_{k}(x)\right\}, U B_{k l} \equiv \min \left\{\left(r_{k l}(x)+\widetilde{Q}\right) /\left(p_{k}(x)-\min \left\{\widetilde{Q}, p_{k}(x)\right\}\right), 1\right\}$, and

$$
\widetilde{Q}=\left\{\begin{array}{cl}
Q / K & \text { under Assumption } 6 \\
Q / K \operatorname{Pr}(X=x) & \text { otherwise }
\end{array}\right.
$$

Proof First consider the bounds with equal-sized partitions. As in Corollary 6.1, with equal-sized partitions, it must be that

$$
p_{k}^{*}=p_{k}+Q_{3, k}-Q_{4, k}=\frac{1}{K},
$$

implying that $Q_{3, k}=Q_{4, k}$. However, within the subsample with $X=x$, this is no longer the case in general. Now,

$$
p_{k}^{*}=\frac{r_{k l}(x)+Q_{1, k l}(x)-Q_{2, k l}(x)}{p_{k}(x)+Q_{3, k}(x)-Q_{4, k}(x)},
$$

where $p_{k}^{*}(x)=p_{k}(x)+Q_{3, k}(x)-Q_{4, k}(x)$ need not equal $1 / K$. Therefore, we must bound

$$
p_{k}^{*}=\frac{r_{k l}(x)+Q_{1, k l}(x)-Q_{2, k l}(x)}{p_{k}(x)+Q_{3, k}(x)-Q_{4, k}(x)} .
$$

With unequal-sized partitions, this turns out to be also be the formula for $p_{k}^{*}$ since it is already the case that the denominator is not in general equal to $1 / K$.

\section{Case 1: Bounds with equal-sized or unequal-sized partitions under Assumptions} 2 (ii) and 6. 
Assumption 6 implies

$$
\begin{aligned}
p_{k}^{*} & =\frac{r_{k l}(x)+Q_{1, k l}(x)-Q_{2, k l}(x)}{p_{k}(x)+Q_{3, k}(x)-Q_{4, k}(x)} \\
& =\frac{r_{k l}(x)+Q_{1, k l}-Q_{2, k l}}{p_{k}(x)+Q_{3, k}-Q_{4, k}} \\
& =\frac{r_{k l}(x)+Q_{1, k l}-Q_{2, k l}}{p_{k}(x)} .
\end{aligned}
$$

It then follows from Proposition 3 that

$$
\frac{r_{k l}(x)-Q / K}{p_{k}(x)} \leq p_{k l}^{*}(x) \leq \frac{r_{k l}(x)+Q / K}{p_{k}(x)-\min \left\{Q / K, p_{k}(x)\right\}} .
$$

In addition, $p_{k l}^{*}(x) \in[0,1]$ since it is a probability and

$$
p_{k l}^{*}(x) \in\left[1-\sum_{\substack{l^{\prime}=1,2, \ldots, K \\ l^{\prime} \neq l}} U B_{k l^{\prime}}(x), 1-\sum_{\substack{l^{\prime}=1,2, \ldots, K \\ l^{\prime} \neq l}} L B_{k l^{\prime}}(x)\right]
$$

since the rows of $P_{0,1}^{*}(x)$ must sum to one.

Case 2: Bounds with equal-sized or unequal-sized partitions under Assumption 2(ii).

Under Assumption 2(ii),

$$
\begin{aligned}
Q_{j, k l} & =\sum_{x} Q_{j, k l}(x) \operatorname{Pr}(X=x) \leq \frac{Q}{K}, j=1,2 \\
Q_{j, k} & =\sum_{x} Q_{j, k}(x) \operatorname{Pr}(X=x) \leq \frac{Q}{K}, j=3,4
\end{aligned}
$$

This implies

$$
Q_{1, k l}(x), Q_{2, k l}(x), Q_{3, k}(x), Q_{4, k}(x) \leq \min \left\{\frac{Q}{K \operatorname{Pr}(X=x)}\right\}
$$


It then follows from Proposition 3 that

$$
\frac{r_{k l}(x)-Q / K \operatorname{Pr}(X=x)}{p_{k}(x)} \leq p_{k l}^{*}(x) \leq \frac{r_{k l}(x)+Q / K \operatorname{Pr}(X=x)}{p_{k}(x)-\min \left\{Q / K \operatorname{Pr}(X=x), p_{k}(x)\right\}}
$$

In addition, $p_{k l}^{*}(x) \in[0,1]$ since it is a probability and

$$
p_{k l}^{*}(x) \in\left[1-\sum_{\substack{l^{\prime}=1,2, \ldots, K \\ l^{\prime} \neq l}} U B_{k l^{\prime}}(x), 1-\sum_{\substack{l^{\prime}=1,2, \ldots, K \\ l^{\prime} \neq l}} L B_{k l^{\prime}}(x)\right]
$$

since the rows of $P_{0,1}^{*}(x)$ must sum to one.

The proof of sharpness follows from the proof for Proposition 3, replacing the unconditional probabilities with conditional probabilities and utilizing the correct maximum misclassification rate.

\section{Q.E.D.}

Corollary 6.3. Consider a $2 \times 2$ transition matrix, $P_{0,1}^{*}$, with unequal-sized partitions. Under Assumption 3, the four elements are bounded sharply by

$$
\begin{aligned}
& p_{11}^{*}(x) \in \max \left\{\frac{r_{11}(x)}{\min \left\{p_{1}(x)+\widetilde{Q}, 1\right\}}, 1-U B_{12}(x), 0\right\}, \min \left\{\frac{r_{11}(x)+\widetilde{Q}}{p_{1}(x)}, 1-L B_{12}(x), 1\right\} \\
& p_{12}^{*}(x) \in \max \left\{\frac{r_{12}(x)-\widetilde{Q}}{p_{1}(x)}, 1-U B_{11}(x), 0\right\}, \min \left\{\frac{r_{12}(x)+\min \left\{\widetilde{Q}, 1-p_{1}(x)\right\}}{p_{1}(x)+\min \left\{\widetilde{Q}, 1-p_{1}(x)\right\}}, 1-L B_{11}(x), 1\right\} \\
& p_{21}^{*}(x) \in \max \left\{\frac{r_{21}(x)-\min \left\{\widetilde{Q}, p_{2}(x)\right\}}{p_{2}(x)-\min \left\{\widetilde{Q}, p_{2}(x)\right\}}, 1-U B_{22}(x), 0\right\}, \min \left\{\frac{r_{21}(x)+\widetilde{Q}}{p_{2}(x)-\widetilde{\widetilde{Q}}}, 1-L B_{22}(x), 1\right\} \\
& p_{22}^{*}(x) \in \max \left\{\frac{r_{22}(x)-\widetilde{Q}}{p_{2}(x)}, 1-U B_{21}(x), 0\right\}, \min \left\{\frac{r_{22}(x)}{p_{2}(x)-\min \left\{\widetilde{Q}, p_{2}(x)\right\}}, 1-L B_{21}(x), 1\right\}
\end{aligned}
$$

where $L B_{k l}(x)$ and $U B_{k l}(x)$ denote the lower and upper bounds of $p_{k l}^{*}(x)$, respectively,

$$
\widetilde{\widetilde{Q}}=\left\{\begin{array}{cl}
0 & \text { under Assumption } 2(i) \\
\min \left\{\widetilde{Q}, p_{2}(x)\right\} & \text { under Assumption } 2(i i)
\end{array}\right.
$$


and

$$
\widetilde{Q}=\left\{\begin{array}{cl}
Q & \text { under Assumptions 2(i) and } 6 \\
Q / \operatorname{Pr}(X=x) & \text { under Assumption 2(i) } \\
Q / 2 & \text { under Assumptions 2(ii) and } 6 \\
Q / 2 \operatorname{Pr}(X=x) & \text { under Assumption 2(ii) }
\end{array}\right.
$$

Proof Following the proof of Proposition 4, we have

$$
\begin{aligned}
p_{11}^{*}(x) & =\frac{r_{22}(x)+\left[\theta_{11}^{10}(x)+\theta_{11}^{01}(x)+\theta_{11}^{11}(x)\right]}{p_{2}(x)+\left[\theta_{11}^{10}(x)+\theta_{11}^{11}(x)+\theta_{12}^{10}(x)\right]} \\
p_{12}^{*}(x) & =\frac{r_{12}(x)+\theta_{12}^{10}(x)-\theta_{11}^{01}(x)}{p_{1}(x)+\left[\theta_{11}^{10}(x)+\theta_{11}^{11}(x)+\theta_{12}^{10}(x)\right]} \\
p_{21}^{*}(x) & =\frac{r_{21}(x)+\theta_{21}^{01}(x)-\theta_{11}^{10}(x)}{p_{2}(x)-\left[\theta_{11}^{10}(x)+\theta_{11}^{11}(x)+\theta_{12}^{10}(x)\right]} \\
p_{22}^{*}(x) & =\frac{r_{22}(x)-\left[\theta_{11}^{11}(x)+\theta_{12}^{10}(x)+\theta_{21}^{01}(x)\right]}{p_{2}(x)-\left[\theta_{11}^{10}(x)+\theta_{11}^{11}(x)+\theta_{12}^{10}(x)\right]} .
\end{aligned}
$$

Under Assumption 6, $\theta_{k, l}^{k^{\prime}-k, l^{\prime}-l}(x)=\theta_{k, l}^{k^{\prime}-k, l^{\prime}-l} \forall k, l, k^{\prime}, l^{\prime}$. Thus, we have

$$
\begin{aligned}
p_{11}^{*}(x) & =\frac{r_{22}(x)+\left(\theta_{11}^{10}+\theta_{11}^{01}+\theta_{11}^{11}\right)}{p_{2}(x)+\left(\theta_{11}^{10}+\theta_{11}^{11}+\theta_{12}^{10}\right)} \\
p_{12}^{*}(x) & =\frac{r_{12}(x)+\theta_{12}^{10}-\theta_{11}^{01}}{p_{1}(x)+\left(\theta_{11}^{10}+\theta_{11}^{11}+\theta_{12}^{10}\right)} \\
p_{21}^{*}(x) & =\frac{r_{21}(x)+\theta_{21}^{01}-\theta_{11}^{10}}{p_{2}(x)-\left(\theta_{11}^{10}+\theta_{11}^{11}+\theta_{12}^{10}\right)} \\
p_{22}^{*}(x) & =\frac{r_{22}(x)-\left(\theta_{11}^{11}+\theta_{12}^{10}+\theta_{21}^{01}\right)}{p_{2}(x)-\left(\theta_{11}^{10}+\theta_{11}^{11}+\theta_{12}^{10}\right)} .
\end{aligned}
$$

\section{Case 1: Bounds under Assumptions 2(i) and 6.}

Following Proposition 4, we have

$$
\begin{aligned}
\frac{r_{11}(x)}{\min \left\{p_{1}(x)+Q, 1\right\}} \leq p_{11}^{*}(x) & \leq \min \left\{\frac{r_{11}(x)+Q}{p_{1}(x)}, 1\right\} \\
\max \left\{\frac{r_{12}(x)-Q}{p_{1}(x)}, 0\right\} & \leq p_{12}^{*}(x) \leq \min \left\{\frac{r_{12}(x)+\min \left\{Q, 1-p_{1}(x)\right\}}{p_{1}(x)+\min \left\{Q, 1-p_{1}(x)\right\}}, 1\right\} \\
\max \left\{\frac{r_{21}(x)-\min \left\{Q, p_{2}(x)\right\}}{p_{2}(x)-\min \left\{Q, p_{2}(x)\right\}}, 0\right\} & \leq p_{21}^{*}(x) \leq \min \left\{\frac{r_{21}(x)+Q}{p_{2}(x)}, 1\right\} \\
\max \left\{\frac{r_{22}(x)-Q}{p_{2}(x)}, 0\right\} & \leq p_{22}^{*}(x) \leq \min \left\{\frac{r_{22}(x)}{p_{2}(x)-\min \left\{Q, p_{2}(x)\right\}}, 1\right\} .
\end{aligned}
$$




\section{Case 2: Bounds under Assumptions 2(ii) and 6.}

Following Proposition 4, we have

$$
\begin{aligned}
\frac{r_{11}(x)}{\min \left\{p_{1}(x)+Q / 2,1\right\}} \leq p_{11}^{*}(x) & \leq \min \left\{\frac{r_{11}(x)+Q / 2}{p_{1}(x)}, 1\right\} \\
\max \left\{\frac{r_{12}(x)-Q / 2}{p_{1}(x)}, 0\right\} \leq p_{12}^{*}(x) & \leq \min \left\{\frac{r_{12}(x)+\min \left\{Q / 2,1-p_{1}(x)\right\}}{p_{1}(x)+\min \left\{Q / 2,1-p_{1}(x)\right\}}, 1\right\} \\
\max \left\{\frac{r_{21}(x)-\min \left\{Q / 2, p_{2}(x)\right\}}{p_{2}(x)-\min \left\{Q / 2, p_{2}(x)\right\}}, 0\right\} \leq p_{21}^{*}(x) & \leq \min \left\{\frac{r_{21}(x)+Q / 2}{p_{2}(x)-\min \left\{Q / 2, p_{2}(x)\right\}}, 1\right\} \\
\max \left\{\frac{r_{22}(x)-Q / 2}{p_{2}(x)}, 0\right\} \leq p_{22}^{*}(x) & \leq \min \left\{\frac{r_{22}(x)}{p_{2}(x)-\min \left\{Q / 2, p_{2}(x)\right\}}, 1\right\} .
\end{aligned}
$$

\section{Case 3: Bounds under Assumptions 2(i).}

This is identical to Case 1 , with $Q$ replaced by $Q / \operatorname{Pr}(X=x)$, following Corollary 6.1 and 6.2 .

\section{Case 4: Bounds under Assumptions 2(ii).}

This is identical to Case 1 , with $Q / K$ replaced by $Q / K \operatorname{Pr}(X=x)$, following Corollary 6.1 and 6.2 .

The proof of sharpness follows from the proof for Proposition 4, replacing the unconditional probabilities with conditional probabilities and utilizing the correct maximum misclassification rate.

Q.E.D. 


\section{Simulated Misclassification Rates}

The total misclassification rate in income data, $Q$, is unknown, but figures prominently in the width of the bounds. While one of the advantages of our approach is that the transition probabilities may be bounded under various assumptions concerning $Q$, having some prior knowledge concerning plausible values of $Q$ is important. Here, we discuss one possible way to obtain reasonable choices of $Q$.

Prior to discussing our approach, it is worth noting that some previous studies have attempted to quantify the measurement error rate in income data by matching self-reported income to administrative data. For example, Pedace and Bates (2000) compare self-reported earnings for over 50,000 respondents in the 1992 SIPP longitudinal file to respondents' earnings documented in the Social Security Administration's Summary Earnings Record (SER). The authors find that $3.6 \%(6.4 \%)$ of the final sample report no (positive) earnings in the SIPP despite having positive (no) earnings in the SER. A similar exercise in Kapteyn and Ypma (2007) using Swedish data on older individuals finds that 18.2\% (4.6\%) report no (pos-

itive) earnings in the survey data despite having positive (no) earnings in the administrative records. While interesting, such results are of limited usefulness in the current context of estimating transition probabilities since rates of measurement error in self-reported earnings do not directly translate into misclassification rates when examining transition matrices. Moreover, the amount of misclassification generated by a given amount of measurement error will depend on the dimensionality of the transition matrix.

In light of this, we employ a simulation-based approach to quantify $Q$ when the data is discretized into a different number of equal-sized partitions. To proceed, we utilize a structural model for income dynamics and measurement error based on an $A R(1)$ model as in Lee et al. (2017). Note, while Lee et al. (2017) rely on the model to assess mobility, we only rely on the model to suggest plausible values of the misclassification rate, $Q$.

The model for income dynamics is given by

$$
\ln \left(y_{i t}^{*}\right)=\gamma \ln \left(y_{i, t-1}^{*}\right)+\alpha_{i}+\varepsilon_{i t}, \quad t=1, \ldots, T
$$

where $y_{i t}^{*}$ is the true income of household $i$ at time $t, y_{i, t-1}^{*}$ is the true income at time $t-1$, 
$\alpha_{i}$ is a time invariant household-specific intercept, and $\varepsilon_{i t}$ is an idiosyncratic error term.

The initial condition is drawn from the stationary distribution of $y$, given by

$$
\ln \left(y_{i 0}^{*}\right) \stackrel{\mathrm{iid}}{\sim} \mathbb{N}\left(\frac{\alpha_{i}}{1-\gamma}, \frac{\sigma_{\alpha}^{2}}{(1-\gamma)^{2}}+\frac{\sigma_{\varepsilon}^{2}}{1-\gamma^{2}}\right)
$$

where $\sigma_{\alpha}^{2}$ and $\sigma_{\varepsilon}^{2}$ are the variances of $\alpha$ and $\varepsilon$, respectively. The fixed effect, $\alpha$, is drawn from a uniform distribution, given by

$$
\alpha_{i} \stackrel{\text { iid }}{\sim} \mathbb{U}\left(\alpha_{l}, \alpha_{u}\right)
$$

The mean of $\alpha, \bar{\alpha}=0.5\left(\alpha_{l}+\alpha_{u}\right)$, is chosen to pin down the expected value of $\ln \left(y^{*}\right)$ for a given choice of $\gamma$ as

$$
\mathrm{E}\left[\ln \left(y^{*}\right)\right]=\frac{\bar{\alpha}}{1-\gamma}
$$

assuming $\mathrm{E}\left[\varepsilon_{i t}\right]=0$. The bounds on $\alpha$ are given by

$$
\begin{aligned}
\alpha_{l} & =\bar{\alpha}-\kappa \\
\alpha_{u} & =\bar{\alpha}+\kappa .
\end{aligned}
$$

implying that $\sigma_{\alpha}^{2}=\kappa^{2} / 3$. The idiosyncratic error, $\varepsilon$, is drawn from a normal distribution, given by

$$
\varepsilon_{i t} \stackrel{\operatorname{iid}}{\sim} \mathbb{N}\left(0, \sigma_{\varepsilon}^{2}\right)
$$

The variance of $\varepsilon$ is chosen to pin down the variance of $\ln \left(y^{*}\right)$ for a given choice of $\gamma$ as given in $(\mathrm{D} .2)$.

We do not observe true income, $y_{i t}^{*}$, but rather mismeasured income, $y_{i t}$. Given that there is substantial evidence that measurement error is mean-reverting and serially correlated (Bound and Krueger 1991; Bound et al. 1994; Bollinger and Chandra 2005; Kim and Solon 2005), we express observed income as

$$
\ln \left(y_{i t}\right)=\rho \ln \left(y_{i t}^{*}\right)+\eta_{i t},
$$


where $\rho$ denotes the mean reversion parameter, $\rho \in(0,1)$. The error, $\eta_{i t}$, is assumed to be indepedent of $y^{*}$ and is decomposed into a time invariant and time-varying component as follows

$$
\eta_{i t}=e_{i}+v_{i t}
$$

where $\operatorname{Cov}\left(e_{i}, v_{i t}\right)=0$ and $e$ and $v$ are each normally distributed.

Define the realized measurement error as

$$
\begin{aligned}
\mu_{i t} & =\ln \left(y_{i t}\right)-\ln \left(y_{i t}^{*}\right) \\
& =(\rho-1) \ln \left(y_{i t}^{*}\right)+\eta_{i t},
\end{aligned}
$$

where

$$
\begin{aligned}
\mathrm{E}\left[\mu_{i t}\right] & =(\rho-1) \mathrm{E}\left[\ln \left(y^{*}\right)\right]+\mathrm{E}\left[\eta_{i t}\right] \\
\operatorname{Var}\left(\mu_{i t}\right) & =(\rho-1)^{2} \operatorname{Var}\left[\ln \left(y^{*}\right)\right]+\operatorname{Var}\left[\eta_{i t}\right]
\end{aligned}
$$

The mean and variance of $\eta$ are chosen to pin down the mean and the variance of $\mu$ for a given choice of $\rho$. Finally, we set

$$
\begin{aligned}
\mathrm{E}\left[e_{i}\right] & =0 \\
\mathrm{E}\left[v_{i t}\right] & =\mathrm{E}\left[\mu_{i t}\right]-(\rho-1) \mathrm{E}\left[\ln \left(y^{*}\right)\right] \\
\sigma_{e}^{2} & =\zeta\left(\operatorname{Var}\left(\mu_{i t}\right)-(\rho-1)^{2} \operatorname{Var}\left[\ln \left(y^{*}\right)\right]\right) \\
\sigma_{v}^{2} & =(1-\zeta)\left(\operatorname{Var}\left(\mu_{i t}\right)-(\rho-1)^{2} \operatorname{Var}\left[\ln \left(y^{*}\right)\right]\right)
\end{aligned}
$$

where $\sigma_{e}^{2}$ and $\sigma_{v}^{2}$ are the variances of $e$ and $v$, respectively.

In sum, we need to choose values for $\gamma$ (auto-regressive parameter in (D.1)), $\rho$ (meanreverting parameter in (D.4) $), \kappa$ (variance of fixed effect in $(\mathrm{D} .3)$ ) $\zeta$ (share of measurement error variance due to time invariant component in $(\mathrm{D} .9)$ ), and $\mathrm{E}\left[\mu_{i t}\right]$ and $\operatorname{Var}\left(\mu_{i t}\right)$ (mean and variance of measurement error in (D.6)). However, instead of directly choosing values for $\operatorname{Var}\left(\mu_{i t}\right)$, we instead choose values for the reliability statistic (Gottschalk and Huynh 2010; 
Abowd and Stinson 2013), given as

$$
R=\frac{\operatorname{Var}\left[\ln \left(y^{*}\right)\right]}{\operatorname{Var}\left[\ln \left(y^{*}\right)\right]+\operatorname{Var}\left(\mu_{i t}\right)} .
$$

The remaining parameters are chosen to pin down $\mathrm{E}\left[\ln \left(y^{*}\right)\right]$ and $\operatorname{Var}\left[\ln \left(y^{*}\right)\right]$ (mean and variance of true income in (D.2) $)$.

We consider the following cases

$$
\begin{aligned}
\mathrm{E}\left[\ln \left(y^{*}\right)\right] & =10 \\
\operatorname{Var}\left[\ln \left(y^{*}\right)\right] & =2 \\
\mathrm{E}\left[\mu_{i t}\right] & =-0.15 \\
(\gamma, \kappa) & =\{(0.9,0.2),(0.8,0.45),(0.7,0.7),(0.6,0.95)\} \\
(R, \rho) & =\{(0.95,0.8),(0.9,0.7),(0.85,0.6),(0.8,0.55)\} \\
\zeta & =\{0.25,0.50,0.75\}
\end{aligned}
$$

Note, $\gamma$ and $\kappa$ are chosen together to enure that $\sigma_{\varepsilon}^{2}>0$ and $R$ and $\rho$ are chosen together to ensure that $\sigma_{e}^{2}, \sigma_{v}^{2}>0$.

The mean and the variance of $\ln \left(y^{*}\right)$ are set at values a bit above those observed in our self-reported income data. The mean measurement error, $\mathrm{E}\left[\mu_{i t}\right]=-0.15$, is from Gottschalk and Huynh (2010) and is based on annual earnings of males in the 1996 SIPP panel matched to tax records and assumes the tax records are correct. In contrast, Gibson and Kim (2010) use the Panel Study of Income Dynamics Validation Study (PSIDVS) based on 1981-1986 data and find the mean measurement error to range from -0.22 to 0.22 across the different years. However, in results not shown, $Q$ is not particularly sensitive to this value.

For the reliability statistic, $R$, Gottschalk and Huynh (2010) and Abowd and Stinson (2013) both report values around 0.7 when assuming the adminstrative data are correct. However, when Abowd and Stinson (2013) give equal probability to the SIPP and administrative data being correct, the reliability statistic of the SIPP data rises to above 0.9. Hyslop and Townsend (2016) perform a similar analysis using survey and administrative data from New Zealand and report reliability statistics between 0.83 and 0.85 . Thus, we consider values 
of $R$ from 0.8 to 0.95 . These values for $R$ then allow us to consider values of $\rho$ from 0.55 to 0.8. This is a reasonable range based on the literature. Specifically, Bound and Krueger (1991) and Bollinger (1998) examine the structure of response error when income is the natural log of annual labor market earnings using the Social Security income data matched to the 1977 and 1978 Current Population Survey. The authors obtain estimates of $\rho$ roughly equal to 0.90. However, using the PSIDVS, Gibson and Kim (2010) find much lower values, with $\rho$ ranging from 0.3 to 0.7 . Kim and Solon (2005) discuss a number of estimates from prior studies, citing values between roughly 0.6 and 0.8 .

For the autoregressive parameter, $\gamma$, we consider a range from 0.6 to 0.9. Gustavsson and Österholm (2014) use Swedish longitudinal data on males observed from 1968-2005 to estimate individual-level time series models of income dynamics. The authors obtain median values of $\gamma$ ranging from 0.6 to 0.8 across samples and specifications. Finally, without any prior information, we consider a range of values of $\zeta$ from 0.25 to 0.75 .

With four values of $(\gamma, \kappa)$, four values of $(R, \rho)$, and three values of $\zeta$, we end up with 48 total cases. For each case, we simulate 1000 data sets with $N=10,000$ and $T=100$. We then retain periods $t=97,100$ in order compute transition matrices over four periods. The choice of $N$ is comparable to the data in our application. The choice of $T$ ensures that initial conditions play a limited role. The choice of a four-year transition matrix follows from our application. Finally, we compute the misclassification rate in each of the 1000 data sets for different dimensions of the transition matrix, $K=2, \ldots, 5$. Denote this misclassification rate as $Q(K)$.

Table D1 reports the mean misclassification rates. The following findings stand out. First, for any value of the parameters, $Q(K)$ unsurprisingly increases significantly with $K$. Second, for any given combination of $\gamma, \kappa$, and $\zeta, Q(K)$ increases significantly as $R$ and $\rho$ decrease. This is not surprising since $R$ directly controls the salience of the measurement error. Howvever, the misclassification rate does not increase linearly across the columns (for a given $\zeta$ ). In particular, the misclassification rate increases by roughly $50 \%$ as $R$ falls from 0.95 to 0.9 , but then only rises marginally as $R$ falls from 0.9 to 0.85 . The misclassification rate then increases by roughly $70 \%$ as $R$ falls from 0.85 to 0.80 . Third, for any given $K$, $Q(K)$ is marginally decreasing with $\zeta$. This holds for all values of $\gamma$ and $\rho$. This implies that 
misclassification is less likely to occur when the time invariant component of the measurement error, $\eta$, is more salient. Finally, for any given combination of $R, \rho$, and $\zeta, Q(K)$ decreases as one moves from Panel A to D. As one moves from Panel A to D, $\gamma$ declines and $\kappa$ increases. As $\gamma$ declines, $\operatorname{Var}\left[\ln \left(y^{*}\right)\right]$ declines as shown in (D.2). Because we wish to hold $\operatorname{Var}\left[\ln \left(y^{*}\right)\right]$ constant (and equal to two), $\sigma_{\alpha}^{2}$ and/or $\sigma_{\varepsilon}^{2}$ must increase. The corresponding rise in $\kappa$ ensures that some of the increase is due to the fixed effect, $\alpha$. The fall in the misclassification rate across the panels of the table implies that misclassification is less likely to occur when income shocks are less permanent.

As it relates to our application, the assumption of $Q=0.20$ seems reasonable in the case of tercile transition matrices. The corresponds to roughly the maximum misclassification rate when $K=3$ and the reliability statistic is at least 0.85 . In the case of poverty transition matrices, we use $Q=0.10$. This corresponds to roughly the maximum misclassification rate when $K=2$ and the reliability statistic is at least 0.85 .

\section{References}

[1] Abowd, J.M. and M.H. Stinson (2013), "Estimating Measurement Error in Annual Job Earnings: A Comparison of Survey and Administrative Data," Review of Economics and Statistics, 95, 1451-1467.

[2] Bollinger, C.R. (1998), "Measurement error in the current population survey: A nonparametric look," Journal of Labor Economics, 16, 57-71.

[3] Bollinger, C.R. and A. Chandra (2005), "Iatrogenic Specification Error: A Cautionary Tale of Cleaning Data," Journal of Labor Economics, 23, 235-257.

[4] Bound, J., C. Brown, G.J. Duncan, and W.L. Rodgers (1994), "Evidence on the Validity of Cross-Sectional and Longitudinal Labor Market Data," Journal of Labor Economics, $12,345-368$.

[5] Bound, J. and A.B. Krueger (1991), "The Extent of Measurement Error in Longitudinal Earnings data: Do Two Wrongs Make a Right?," Journal of Labor Economics, 9, 1-24. 
[6] Gibson, J. and B. Kim (2010), "Non-Classical Measurement Error in Long-Term Retrospective Recall Surveys," Oxford Bulletin of Economics and Statistics, 72, 687-695.

[7] Gottschalk, P. and M. Huynh (2010), “Are Earnings Inequality and Mobility Overstated? The Impact of Nonclassical Measurement Error," Review of Economics and Statistics, 92, 302-315.

[8] Hyslop, D. and W. Townsend (2016), "Earnings Dynamics and Measurement Error in Matched Survey and Administrative Data," Motu Working Paper 16-18, October.

[9] Kapteyn, A. and J.Y. Ypma (2007), "Measurement Error and Misclassification: A Comparison of Survey and Administrative Data," Journal of Labor Economics, 25, 513-551.

[10] Kim, B. and G. Solon (2005), "Implications of Mean-Reverting Measurement Error for Longitudinal Studies of Wages and Employment," Review of Economics and Statistics, 87, 193-196.

[11] Lee, N., G. Ridder, and J. Strauss (2017), "Estimation of Poverty Transition Matrices with Noisy Data," Journal of Applied Econometrics, 32, 37-55.

[12] Pedace, R. and N. Bates (2000), "Using Administrative Records to Assess Earnings Reporting Error in the Survey of Income and Program Participation," Journal of Economic and Social Measurement, 26, 173-192. 
Panel A. $(\gamma, \kappa)=(0.90,0.20)$

$(\mathbf{R}, \rho)=(0.95,0.80)$

\begin{tabular}{ccc}
\hline$\zeta=\mathbf{0 . 2 5}$ & $\zeta=\mathbf{0 . 5}$ & $\zeta=\mathbf{0 . 7 5}$ \\
\hline 0.074 & 0.073 & 0.073
\end{tabular}

$\mathrm{Q}(3)$

$\mathrm{Q}(4)$

$\mathrm{Q}(5)$

$0.141 \quad 0.140 \quad 0.138$

\begin{tabular}{ccc}
\multicolumn{3}{c}{$(\mathbf{R , \rho )}=(\mathbf{0 . 9 0 , 0 . 7 0 )}$} \\
\hline$\zeta=\mathbf{0 . 2 5}$ & $\boldsymbol{\zeta = 0 . 5}$ & $\boldsymbol{\zeta = 0 . 7 5}$ \\
\hline 0.106 & 0.105 & 0.104 \\
0.201 & 0.199 & 0.196 \\
0.285 & 0.282 & 0.276 \\
0.360 & 0.355 & 0.346
\end{tabular}

\begin{tabular}{ccc}
\multicolumn{3}{c}{$(\mathbf{R}, \boldsymbol{\rho})=(\mathbf{0 . 8 5 , 0 . 6 0 )}$} \\
\hline$\zeta=\mathbf{0 . 2 5}$ & $\boldsymbol{\zeta}=\mathbf{0 . 5}$ & $\boldsymbol{\zeta}=\mathbf{0 . 7 5}$ \\
\hline 0.109 & 0.108 & 0.107 \\
0.207 & 0.205 & 0.201 \\
0.293 & 0.289 & 0.283 \\
0.369 & 0.364 & 0.355
\end{tabular}

\begin{tabular}{ccc}
\multicolumn{3}{c}{$(\mathbf{R , \rho})=(\mathbf{0 . 8 0 , 0 . 5 5 )}$} \\
\hline$\zeta=\mathbf{0 . 2 5}$ & $\zeta=\mathbf{0 . 5}$ & $\zeta=\mathbf{0 . 7 5}$ \\
\hline 0.191 & 0.187 & 0.182 \\
0.350 & 0.343 & 0.332 \\
0.474 & 0.464 & 0.448 \\
0.570 & 0.559 & 0.538
\end{tabular}

Panel B. $(\gamma, \kappa)=(0.80,0.45)$

\begin{tabular}{|c|c|c|c|c|c|c|c|c|c|c|c|c|}
\hline & \multicolumn{3}{|c|}{$(R, \rho)=(0.95,0.80)$} & \multicolumn{3}{|c|}{$(R, \rho)=(0.90,0.70)$} & \multicolumn{3}{|c|}{$(R, \rho)=(0.85,0.60)$} & \multicolumn{3}{|c|}{$(R, \rho)=(0.80,0.55)$} \\
\hline & $\zeta=0.25$ & $\zeta=0.5$ & $\zeta=0.75$ & $\zeta=0.25$ & $\zeta=0.5$ & $\zeta=0.75$ & $\zeta=0.25$ & $\zeta=0.5$ & $\zeta=0.75$ & $\zeta=0.25$ & $\zeta=0.5$ & $\zeta=0.75$ \\
\hline $\mathrm{Q}(2)$ & 0.067 & 0.066 & 0.065 & 0.096 & 0.095 & 0.094 & 0.099 & 0.098 & 0.096 & 0.174 & 0.170 & 0.165 \\
\hline $\mathrm{Q}(3)$ & 0.132 & 0.131 & 0.130 & 0.190 & 0.188 & 0.185 & 0.195 & 0.193 & 0.190 & 0.335 & 0.328 & 0.317 \\
\hline $\mathrm{Q}(4)$ & 0.194 & 0.192 & 0.190 & 0.275 & 0.272 & 0.267 & 0.283 & 0.280 & 0.274 & 0.465 & 0.455 & 0.439 \\
\hline $\mathrm{Q}(5)$ & 0.250 & 0.248 & 0.244 & 0.352 & 0.347 & 0.339 & 0.361 & 0.356 & 0.348 & 0.566 & 0.554 & 0.534 \\
\hline
\end{tabular}

Panel C. $(\gamma, \kappa)=(0.70,0.70)$

$\mathrm{Q}(2)$

$$
(\mathbf{R}, \rho)=(0.95,0.80)
$$

\begin{tabular}{ccc}
\hline$\zeta=\mathbf{0 . 2 5}$ & $\zeta=\mathbf{0 . 5}$ & $\zeta=\mathbf{0 . 7 5}$ \\
\hline 0.064 & 0.063 & 0.063
\end{tabular}

\begin{tabular}{ccc}
\multicolumn{3}{c}{$(\mathbf{R , \rho})=(\mathbf{0 . 9 0 , 0 . 7 0 )}$} \\
\hline$\zeta=\mathbf{0 . 2 5}$ & $\zeta=\mathbf{0 . 5}$ & $\zeta=\mathbf{0 . 7 5}$ \\
\hline 0.092 & 0.091 & 0.089 \\
0.183 & 0.181 & 0.178 \\
0.270 & 0.267 & 0.261 \\
0.349 & 0.344 & 0.336
\end{tabular}

\begin{tabular}{ccc}
\multicolumn{3}{c}{$(\mathbf{R , \rho})=(\mathbf{0 . 8 5 , 0 . 6 0 )}$} \\
\hline$\zeta=\mathbf{0 . 2 5}$ & $\boldsymbol{\zeta}=\mathbf{0 . 5}$ & $\boldsymbol{\zeta}=\mathbf{0 . 7 5}$ \\
\hline 0.095 & 0.093 & 0.092 \\
0.188 & 0.186 & 0.183 \\
0.277 & 0.274 & 0.268 \\
0.358 & 0.352 & 0.344
\end{tabular}

\begin{tabular}{ccc}
\multicolumn{3}{c}{$(\mathbf{R}, \boldsymbol{\rho})=(\mathbf{0 . 8 0 , 0 . 5 5 )}$} \\
\hline$\zeta=\mathbf{0 . 2 5}$ & $\zeta=\mathbf{0 . 5}$ & $\zeta=\mathbf{0 . 7 5}$ \\
\hline 0.167 & 0.163 & 0.157 \\
0.326 & 0.319 & 0.308 \\
0.460 & 0.450 & 0.433 \\
0.564 & 0.552 & 0.531
\end{tabular}

Panel D. $(\gamma, \kappa)=(0.60,0.95)$

\begin{tabular}{|c|c|c|c|c|c|c|c|c|c|c|c|c|}
\hline & \multicolumn{3}{|c|}{$(R, \rho)=(0.95,0.80)$} & \multicolumn{3}{|c|}{$(R, \rho)=(0.90,0.70)$} & \multicolumn{3}{|c|}{$(R, \rho)=(0.85,0.60)$} & \multicolumn{3}{|c|}{$(R, \rho)=(0.80,0.55)$} \\
\hline & $\zeta=0.25$ & $\zeta=0.5$ & $\zeta=0.75$ & $\zeta=0.25$ & $\zeta=0.5$ & $\zeta=0.75$ & $\zeta=0.25$ & $\zeta=0.5$ & $\zeta=0.75$ & $\zeta=0.25$ & $\zeta=0.5$ & $\zeta=0.75$ \\
\hline $\mathrm{Q}(2)$ & 0.062 & 0.062 & 0.061 & 0.089 & 0.088 & 0.086 & 0.092 & 0.091 & 0.089 & 0.163 & 0.158 & 0.152 \\
\hline $\mathrm{Q}(3)$ & 0.124 & 0.123 & 0.122 & 0.179 & 0.176 & 0.173 & 0.184 & 0.181 & 0.177 & 0.320 & 0.312 & 0.300 \\
\hline $\mathrm{Q}(4)$ & 0.186 & 0.184 & 0.182 & 0.265 & 0.262 & 0.256 & 0.272 & 0.269 & 0.263 & 0.455 & 0.445 & 0.427 \\
\hline $\mathrm{Q}(5)$ & 0.244 & 0.242 & 0.238 & 0.345 & 0.340 & 0.332 & 0.354 & 0.349 & 0.340 & 0.562 & 0.549 & 0.528 \\
\hline
\end{tabular}

Notes: Each cell gives the mean misclassification rate, $\mathrm{Q}(\cdot)$, across 1000 simulations with $\mathrm{N}=10,000$ for different combinations of $\gamma$ (autoregressive parameter), $\kappa$ (variance of fixed effect), $R$ (reliability ratio), $\rho$ (mean reversion parameter), $\zeta$ (share of measurement error variance due to time invariant component), and dimension of the transition matrix. See Appendix D for further details. 


\section{E Supplemental Tables}



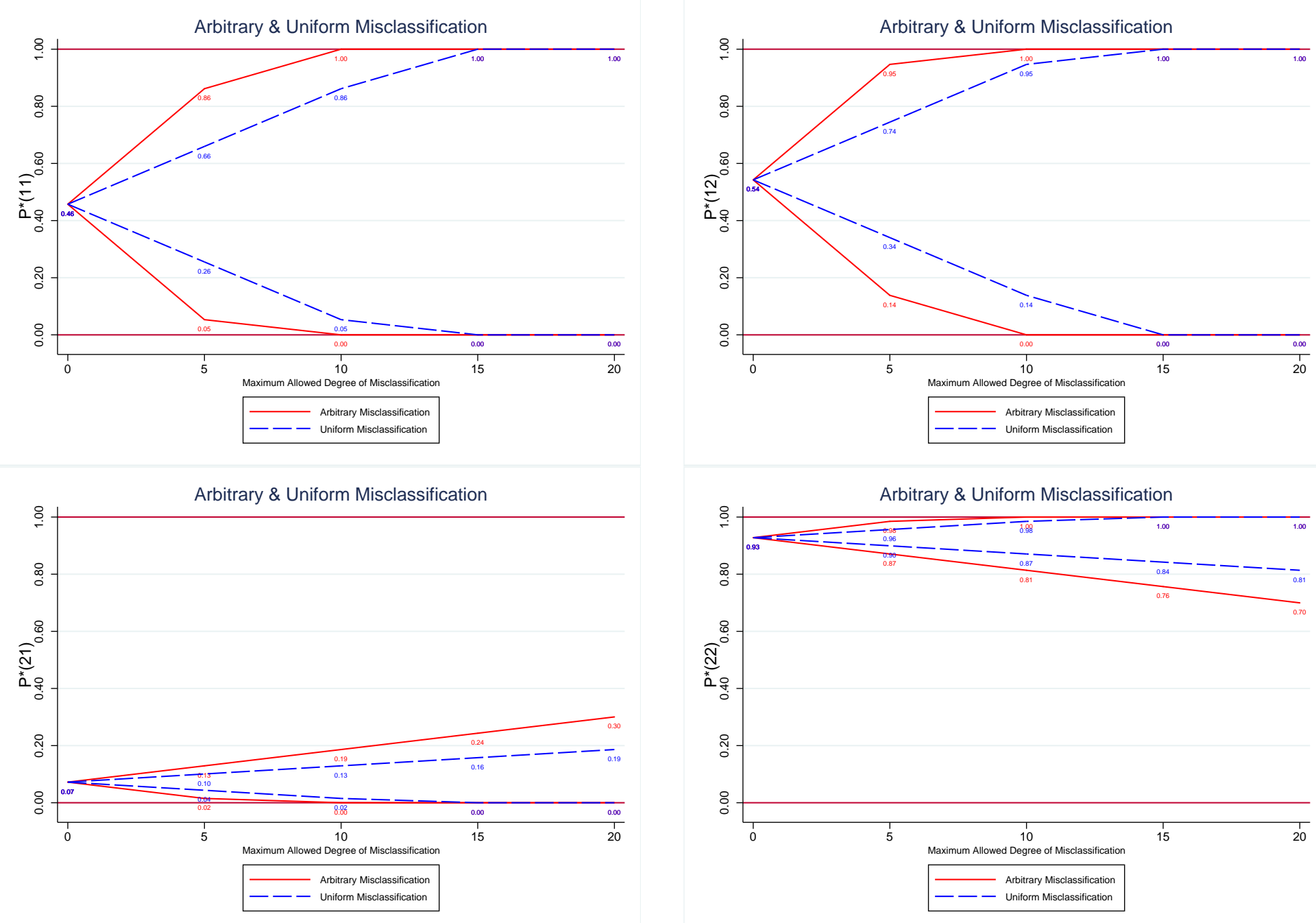

Figure E1. Poverty Transition Matrices Bounds: Sensitivity to $Q$.

Notes: Sample based on pooled 2004-2008 and 2008-2012 panels. See text for further details. 

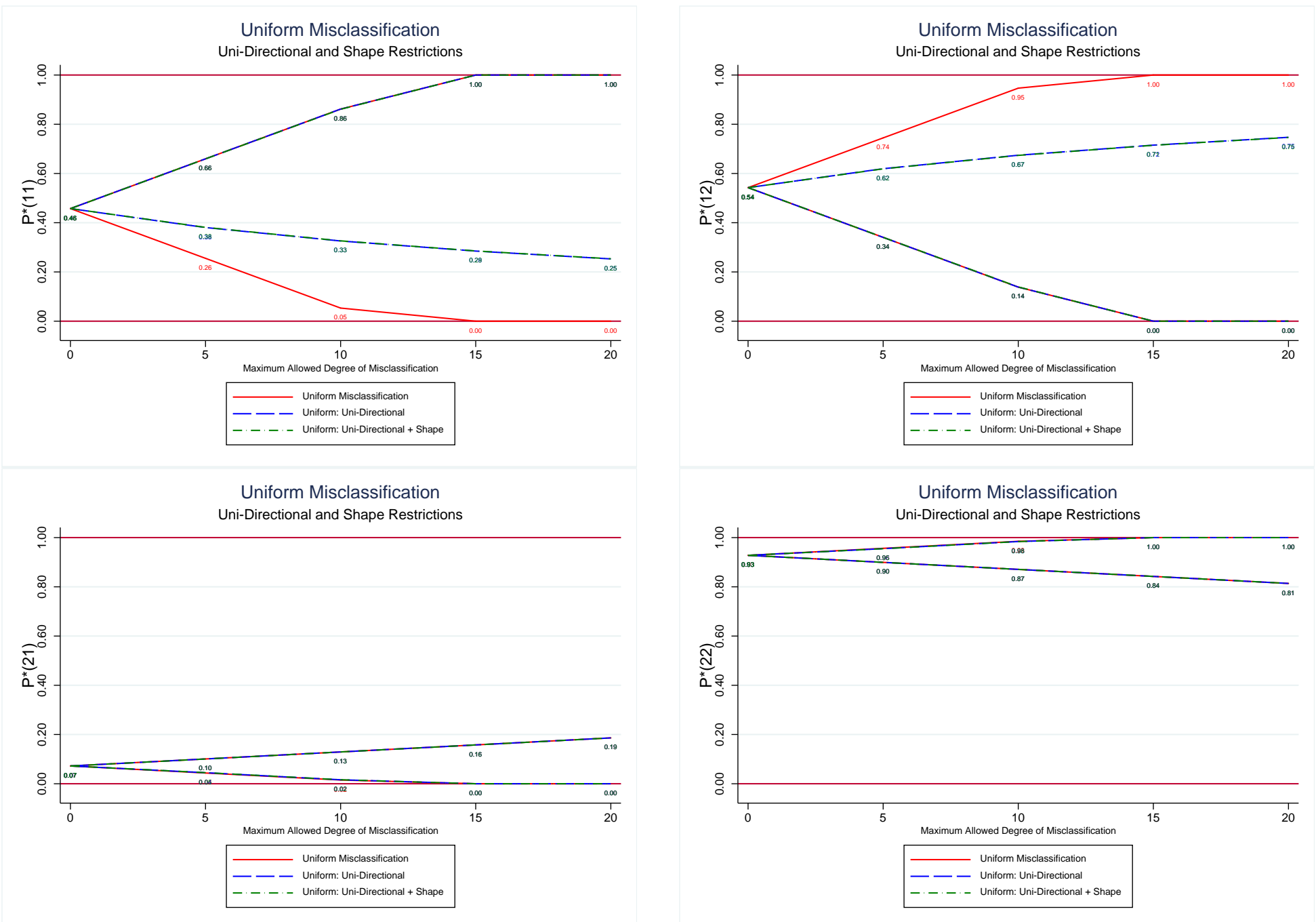

Figure E2. Poverty Transition Matrices Bounds: Sensitivity to $Q$.

Notes: Sample based on pooled 2004-2008 and 2008-2012 panels. Point estimates for bounds obtained using 100 subsamples of size N/2 for bias correction. See text for further details. 

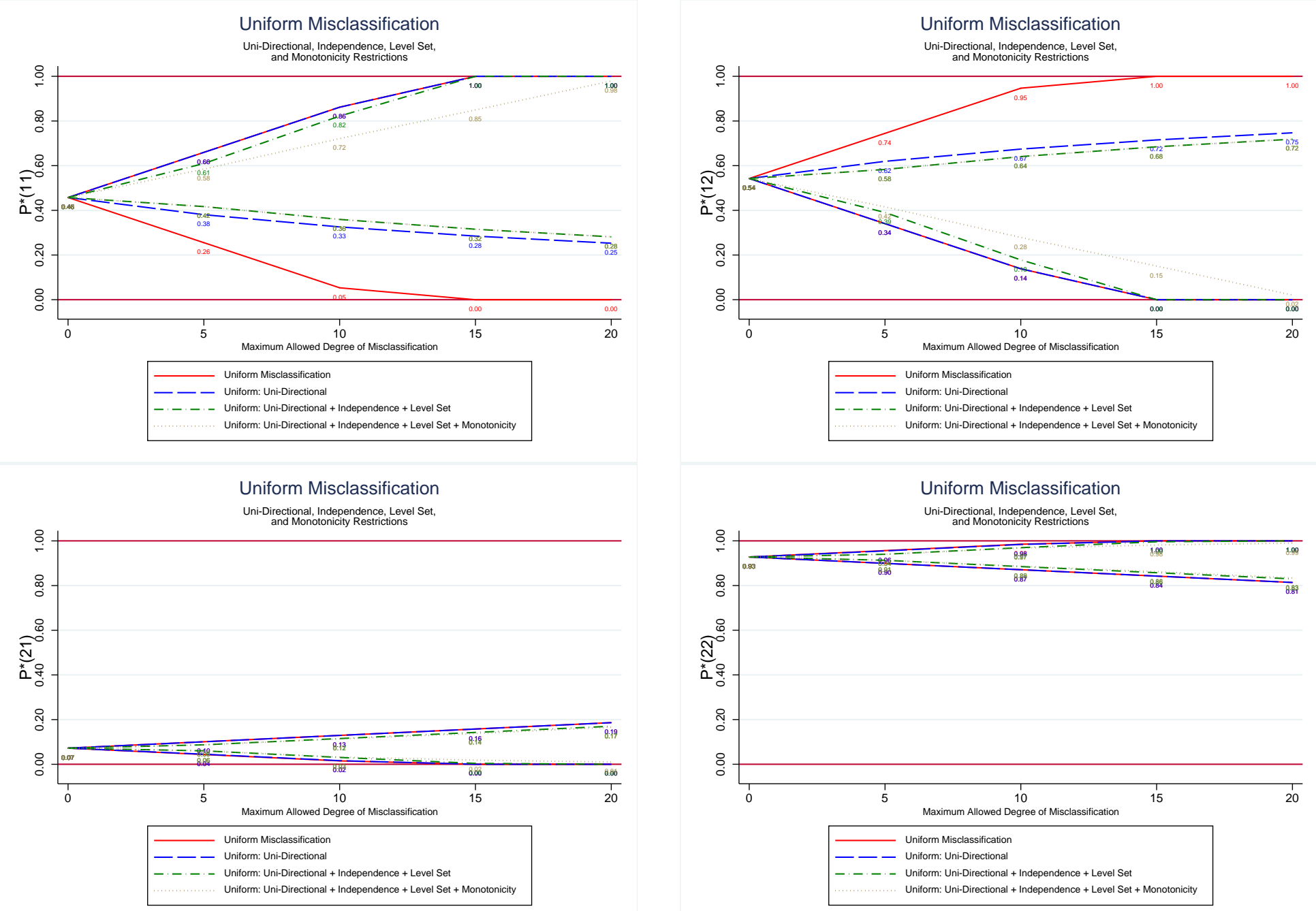

Figure E3. Poverty Transition Matrices Bounds: Sensitivity to $Q$.

Notes: Sample based on pooled 2004-2008 and 2008-2012 panels. Point estimates for bounds obtained using 100 subsamples of size N/2 for bias correction. Level set restrictions based on age of household held using 10-year age intervals and rolling windows of plus/minus one interval both within and across panels. Monotonicity restrictions based on education level of household held using three categories (high school degree and below, some college, and four-year college degree or more). See text for further details. 

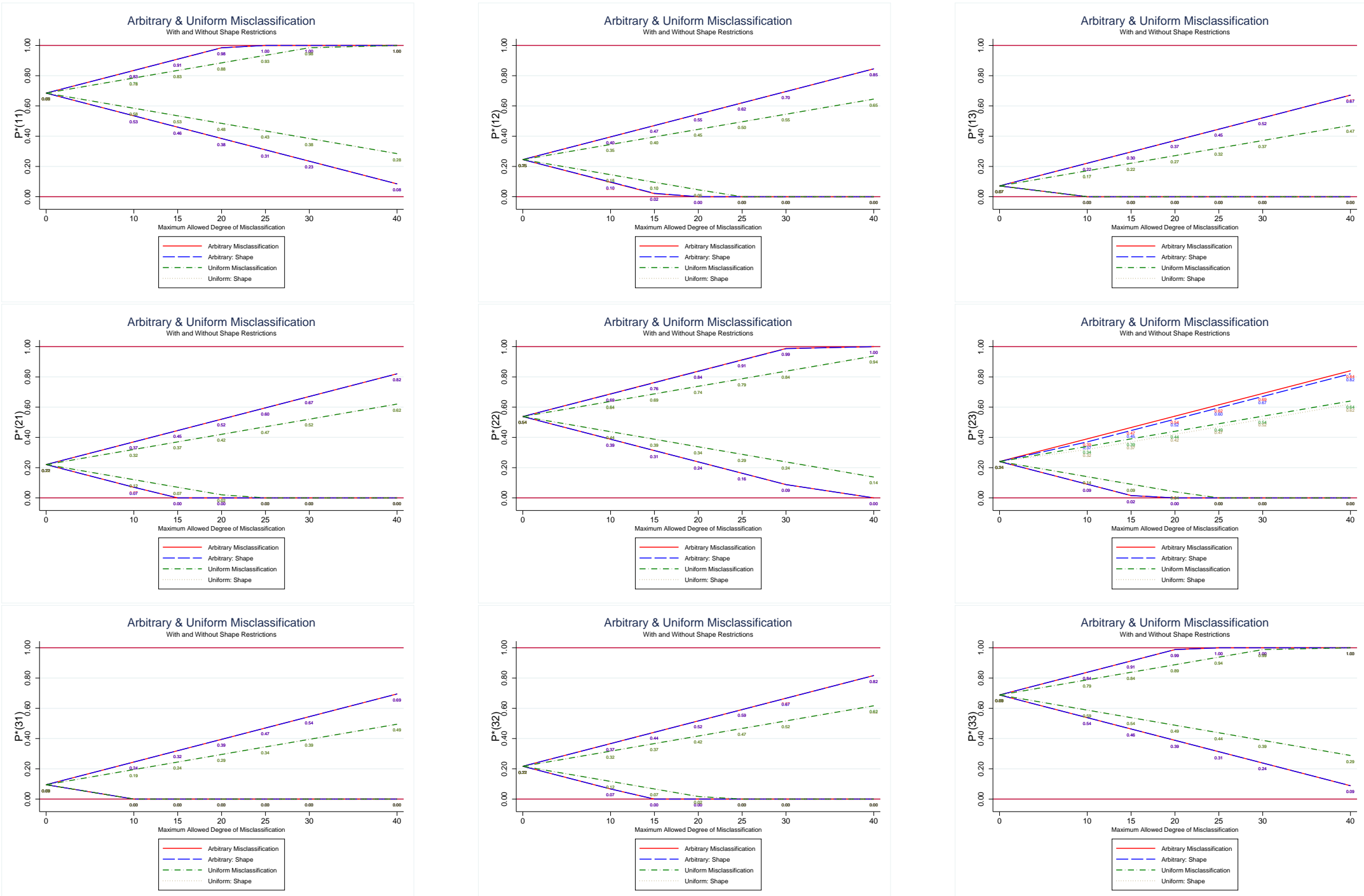

Figure E4. Tercile Transition Matrices Bounds: Sensitivity to $Q$.

Notes: Sample based on pooled 2004-2008 and 2008-2012 panels. Point estimates for bounds obtained using 100 subsamples of size N/2 for bias correction. See text for further details. 

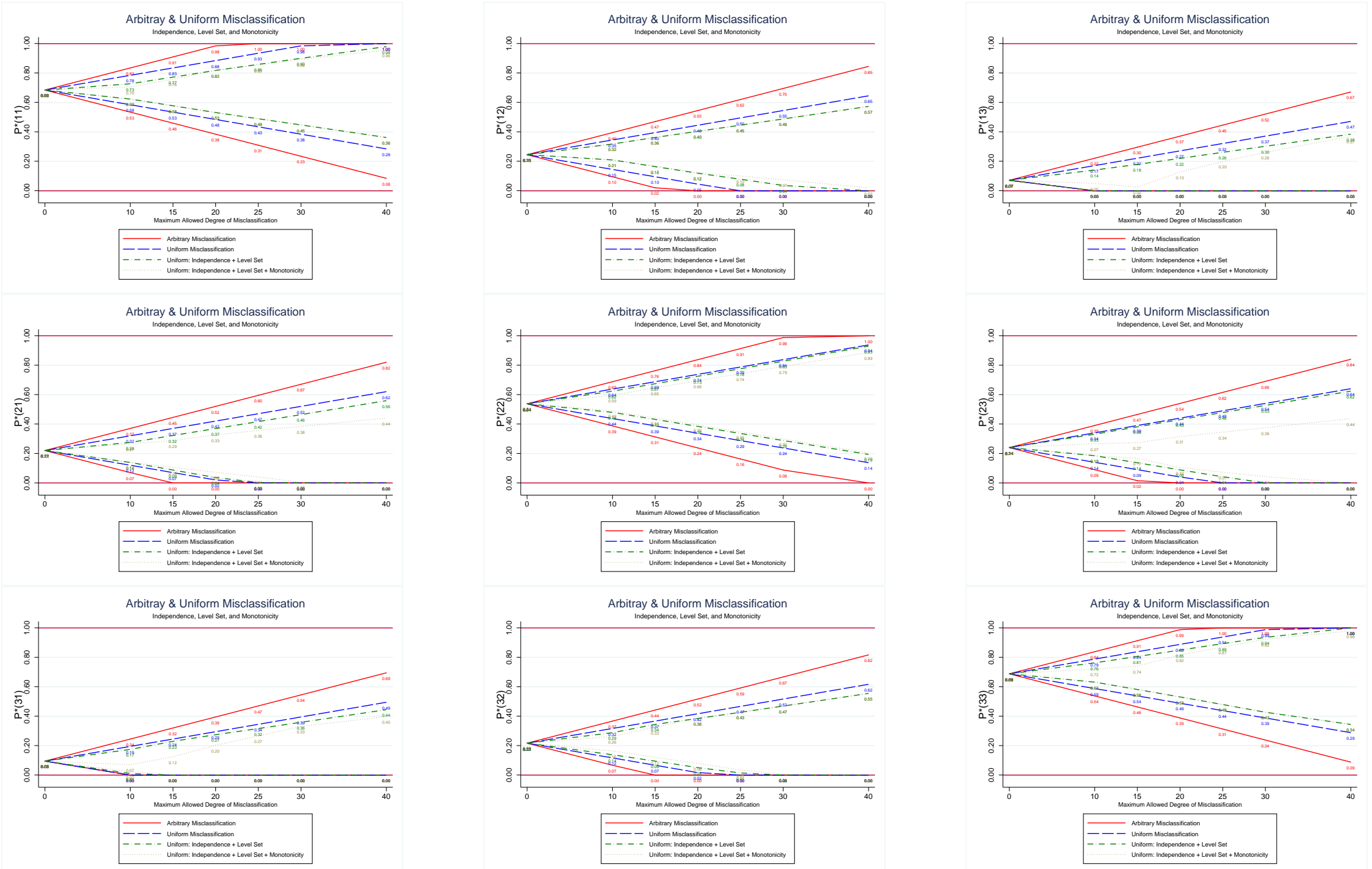

Figure E5. Tercile Transition Matrices Bounds: Sensitivity to $Q$.

Notes: Sample based on pooled 2004-2008 and 2008-2012 panels. Point estimates for bounds obtained using 100 subsamples of size N/2 for bias correction. Level set restrictions based on age of household held using 10-year age intervals and rolling windows of plus/minus one interval both within and across panels. Monotonicity restrictions based on education level of household held using three categories (high school degree and below, some college, and four-year college degree or more). See text for further details. 
Table E1. Tercile Transition Matrices: Misclassification Assumptions.

\section{4-2008 Panel}

I. Classification-Preserving Measurement Error

1

2

3

$1 \quad[0.688,0.688] \quad[0.243,0.243] \quad[0.070,0.070]$ $\begin{array}{lll}(0.676,0.700) & (0.231,0.255) \quad(0.061,0.078)\end{array}$

$2 \quad[0.221,0.221] \quad[0.540,0.540] \quad[0.239,0.239]$ $\begin{array}{lll}(0.210,0.233) & (0.527,0.552) & (0.227,0.250)\end{array}$

$\begin{array}{lllll}3 & {[0.091,0.091]} & {[0.218,0.218]} & {[0.692,0.692]}\end{array}$ $\begin{array}{lll}(0.082,0.100) & (0.207,0.228) \quad(0.680,0.703)\end{array}$

II. Arbitrary Misclassification $(\mathrm{Q}=0.20)$

$$
1
$$$$
2
$$

$1 \quad[0.388,0.988] \quad[0.000,0.543] \quad[0.000,0.370]$ $\begin{array}{lll}(0.379,0.997) & (0.000,0.552) & (0.000,0.376)\end{array}$

$2[0.000,0.521] \quad[0.240,0.840] \quad[0.000,0.539]$ $\begin{array}{llll}(0.000,0.530) & (0.230,0.849) & (0.000,0.548)\end{array}$

$3 \quad[0.000,0.391] \quad[0.000,0.518] \quad[0.392,0.992]$ $\begin{array}{lll}(0.000,0.398) & (0.000,0.526) \quad(0.383,1.000)\end{array}$

III. Uniform Misclassification $(\mathrm{Q}=0.20)$$$
2
$$

3

$1 \quad[0.488,0.888] \quad[0.043,0.443] \quad[0.000,0.270]$ $\begin{array}{llll}(0.479,0.898) & (0.034,0.452) & (0.000,0.276)\end{array}$

$2[0.021,0.421] \quad[0.340,0.740] \quad[0.039,0.439]$ $\begin{array}{llll}(0.012,0.430) & (0.330,0.749) & (0.030,0.448)\end{array}$

$3[0.000,0.291] \quad[0.018,0.418] \quad[0.492,0.892]$ $\begin{array}{lll}(0.000,0.298) & (0.009,0.426) & (0.483,0.900)\end{array}$
$1 \quad[0.687,0.687] \quad[0.241,0.241] \quad[0.076,0.076]$ $\begin{array}{llll}(0.679,0.695) & (0.234,0.249) & (0.070,0.082)\end{array}$

$2 \quad[0.218,0.218] \quad[0.536,0.536] \quad[0.242,0.242]$ $\begin{array}{lll}(0.210,0.225) & (0.528,0.545) & (0.234,0.250)\end{array}$

$3 \quad[0.095,0.095] \quad[0.222,0.222] \quad[0.682,0.682]$ $(0.089,0.102) \quad(0.215,0.230) \quad(0.674,0.691)$

\section{1}

$1[0.387,0.987] \quad[0.000,0.541] \quad[0.000,0.376]$ $\begin{array}{lll}(0.381,0.993) & (0.000,0.547) & (0.000,0.380)\end{array}$

$2[0.000,0.518] \quad[0.236,0.836] \quad[0.000,0.542]$ $\begin{array}{lll}(0.000,0.524) & (0.229,0.843) & (0.000,0.548)\end{array}$

$3 \quad[0.000,0.395] \quad[0.000,0.522] \quad[0.382,0.982]$ $\begin{array}{lll}(0.000,0.400) \quad(0.000,0.528) & (0.376,0.989)\end{array}$
$1[0.487,0.887] \quad[0.041,0.441] \quad[0.000,0.276]$ $\begin{array}{llll}(0.481,0.893) & (0.036,0.447) \quad(0.000,0.280)\end{array}$

$2[0.018,0.418] \quad[0.336,0.736] \quad[0.042,0.442]$ $\begin{array}{lll}(0.012,0.424) & (0.329,0.743) & (0.036,0.448)\end{array}$

$3 \quad[0.000,0.295] \quad[0.022,0.422] \quad[0.482,0.882]$ $\begin{array}{lll}(0.000,0.300) & (0.016,0.428) & (0.476,0.889)\end{array}$
$[0.685,0.685] \quad[0.243,0.243] \quad[0.073,0.073]$ $\begin{array}{lll}(0.677,0.692) & (0.236,0.250) \quad(0.068,0.077)\end{array}$

$2[0.221,0.221] \quad[0.539,0.539] \quad[0.240,0.240]$ $\begin{array}{lll}(0.214,0.227) & (0.532,0.546) & (0.234,0.246)\end{array}$

$3 \quad[0.095,0.095] \quad[0.218,0.218] \quad[0.687,0.687]$ $(0.090,0.100) \quad(0.211,0.224) \quad(0.681,0.694)$

1

$1 \quad[0.385,0.985] \quad[0.000,0.543] \quad[0.000,0.373]$ $\begin{array}{lll}(0.379,0.990) & (0.000,0.548) & (0.000,0.376)\end{array}$

$2[0.000,0.521] \quad[0.239,0.839] \quad[0.000,0.540]$ $\begin{array}{lll}(0.000,0.526) & (0.234,0.845) & (0.000,0.545)\end{array}$

$3 \quad[0.000,0.395] \quad[0.000,0.518] \quad[0.387,0.987]$ $(0.000,0.399) \quad(0.000,0.523) \quad(0.382,0.992)$

\section{1}

2

$1 \quad[0.485,0.885] \quad[0.043,0.443] \quad[0.000,0.273]$ $\begin{array}{llll}(0.479,0.890) & (0.037,0.448) & (0.000,0.276)\end{array}$

$2[0.021,0.421] \quad[0.339,0.739] \quad[0.040,0.440]$ $\begin{array}{llll}(0.016,0.426) & (0.334,0.745) & (0.035,0.445)\end{array}$

$3[0.000,0.295][0.018,0.418] \quad[0.487,0.887]$ $\begin{array}{lll}(0.000,0.299) & (0.013,0.423) & (0.482,0.892)\end{array}$

for the bounds provided in parentheses underneath point estimates obtained using 250 subsamples of size N/2. See text for further details. 
I. No Shape Restrictions

A. Arbitrary, Independent Misclassification $(Q=0.20)$$$
1
$$

2

3

1

2

$1 \quad[0.449,0.968] \quad[0.000,0.490] \quad[0.000,0.331]$ $\begin{array}{lll}(0.433,0.984) & (0.000,0.506) & (0.000,0.342)\end{array}$

$2 \quad[0.000,0.503] \quad[0.273,0.831] \quad[0.000,0.505]$ $\begin{array}{lll}(0.000,0.516) & (0.257,0.847) \quad(0.000,0.520)\end{array}$

$3 \quad[0.000,0.340] \quad[0.000,0.460] \quad[0.462,0.977]$ $\begin{array}{lll}(0.000,0.351) \quad(0.000,0.473) & (0.448,0.989)\end{array}$

B. Uniform, Independent Misclassification $(Q=0.20)$

$$
1
$$

$$
2
$$$$
3
$$

$1 \quad[0.539,0.870] \quad[0.060,0.400] \quad[0.000,0.241]$ $\begin{array}{llll}(0.524,0.886) & (0.046,0.415) & (0.000,0.250)\end{array}$

$2[0.036,0.406] \quad[0.370,0.731] \quad[0.067,0.408]$ $\begin{array}{lll}(0.023,0.418) & (0.354,0.747) & (0.051,0.422)\end{array}$

$3[0.000,0.253][0.018,0.373] \quad[0.549,0.879]$ $(0.000,0.263) \quad(0.009,0.385) \quad(0.536,0.893)$

II. With Shape Restrictions

A. Arbitrary, Independent Misclassification $(Q=0.20)$$$
1
$$$$
2
$$$$
3
$$

$1 \quad[0.449,0.968] \quad[0.000,0.490] \quad[0.000,0.331]$ $[0.433,0.984] \quad[0.000,0.506] \quad[0.000,0.342]$

$2[0.000,0.503][0.273,0.831] \quad[0.000,0.505]$ $[0.000,0.516] \quad[0.257,0.847] \quad[0.000,0.520]$

$3[0.000,0.340] \quad[0.000,0.460] \quad[0.462,0.977]$ $[0.000,0.351] \quad[0.000,0.473] \quad[0.448,0.989]$
$1[0.413,0.971] \quad[0.000,0.512] \quad[0.000,0.350]$ $\begin{array}{lll}(0.405,0.981) & (0.000,0.521) & (0.000,0.356)\end{array}$

$2[0.000,0.512] \quad[0.256,0.825] \quad[0.000,0.534]$ $\begin{array}{lll}(0.000,0.521) & (0.245,0.833) & (0.000,0.543)\end{array}$

$3[0.000,0.369][0.000,0.491] \quad[0.414,0.944]$ $\begin{array}{lll}(0.000,0.377) & (0.000,0.501) & (0.404,0.953)\end{array}$
$10.454,0.000$

$1 \quad[0.454,0.913] \quad[0.019,0.478] \quad[0.000,0.310]$ $\begin{array}{lll}(0.437,0.928) & (0.007,0.493) & (0.000,0.319)\end{array}$

$2[0.000,0.478] \quad[0.266,0.832] \quad[0.000,0.518]$ $\begin{array}{lll}(0.000,0.489) & (0.254,0.841) & (0.000,0.528)\end{array}$

$3[0.000,0.359] \quad[0.000,0.478] \quad[0.435,0.940]$ $\begin{array}{lll}(0.000,0.366) & (0.000,0.486) \quad(0.425,0.948)\end{array}$

B. Uniform, Independent Misclassification $(\mathrm{Q}=0.20)$

$1 \quad[0.539,0.870] \quad[0.060,0.400] \quad[0.000,0.241]$ $\begin{array}{lll}(0.524,0.886) & (0.046,0.415) \quad(0.000,0.250)\end{array}$

$2[0.036,0.406] \quad[0.370,0.731] \quad[0.067,0.408]$ $\begin{array}{llll}(0.023,0.418) & (0.354,0.747) & (0.051,0.422)\end{array}$

$3 \quad[0.000,0.253][0.018,0.373] \quad[0.549,0.879]$ $(0.000,0.263) \quad(0.009,0.385) \quad(0.536,0.893)$



$1 \quad[0.506,0.872] \quad[0.056,0.419] \quad[0.000,0.257]$ $\begin{array}{llll}(0.498,0.882) & (0.046,0.427) & (0.000,0.262)\end{array}$

$2[0.021,0.411] \quad[0.356,0.723] \quad[0.052,0.433]$ $\begin{array}{lll}(0.013,0.420) & (0.346,0.732) & (0.044,0.442)\end{array}$

$3[0.000,0.277][0.047,0.399] \quad[0.515,0.851]$

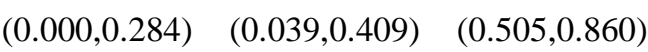

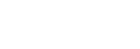

$1[0.538,0.829][0.105,0.394] \quad[0.000,0.228]$ $\begin{array}{llll}(0.522,0.842) & (0.092,0.408) & (0.000,0.236)\end{array}$

$2[0.029,0.381] \quad[0.363,0.730] \quad[0.072,0.419]$ $\begin{array}{llll}(0.021,0.392) & (0.350,0.739) & (0.060,0.429)\end{array}$

$3[0.000,0.272] \quad[0.046,0.389] \quad[0.533,0.853]$ $\begin{array}{lll}(0.000,0.279) \quad(0.039,0.398) & (0.522,0.862)\end{array}$

$\begin{array}{llll} & (0.000,0.284) & (0.039,0.409) & (0.505,0.860)\end{array}$

$1[0.413,0.971][0.000,0.512] \quad[0.000,0.350]$ $[0.405,0.981] \quad[0.000,0.521] \quad[0.000,0.356]$

$2[0.000,0.512] \quad[0.256,0.825] \quad[0.000,0.513]$ $[0.000,0.521] \quad[0.245,0.833] \quad[0.000,0.522]$

$3 \quad[0.000,0.369] \quad[0.000,0.491] \quad[0.414,0.944]$ $[0.000,0.377] \quad[0.000,0.501] \quad[0.404,0.953]$

Notes: Outcome = modified OECD equivalized income. Point estimates for bounds provided in brackets obtained using 100 subsamples of size N/2 for bias correction $90 \%$ Imbens-Manski confidence intervals for the bounds provided in parentheses underneath point estimates obtained using 250 subsamples of size N/2. See Table 3 and text for further details. 
Table E3. Tercile Transition Matrices: Monotonicity + Level Set Restrictions.

I. No Shape Restrictions

A. Arbitrary, Independent Misclassification $(Q=0.20)$$$
1
$$

2

3

1

$1 \quad[0.449,0.948] \quad[0.021,0.490] \quad[0.000,0.283]$ $\begin{array}{llll}(0.433,0.963) & (0.003,0.506) & (0.000,0.306)\end{array}$

$2[0.000,0.390] \quad[0.273,0.815] \quad[0.009,0.362]$ $\begin{array}{llll}(0.000,0.409) \quad(0.257,0.831) & (0.000,0.381)\end{array}$

$3[0.000,0.340] \quad[0.001,0.460] \quad[0.462,0.889]$ $(0.000,0.351) \quad(0.000,0.473) \quad(0.448,0.914)$

B. Uniform, Independent Misclassification $(\mathrm{Q}=0.20)$$$
1
$$$$
2
$$$$
3
$$

$1 \quad[0.539,0.855] \quad[0.080,0.400] \quad[0.000,0.152]$ $\begin{array}{llll}(0.524,0.871) & (0.064,0.415) & (0.000,0.184)\end{array}$

$2 \quad[0.058,0.333] \quad[0.370,0.715] \quad[0.075,0.309]$ $\begin{array}{lll}(0.045,0.350) & (0.354,0.730) & (0.062,0.327)\end{array}$

$3[0.000,0.223][0.056,0.373] \quad[0.549,0.832]$ $\begin{array}{lll}(0.000,0.244) & (0.040,0.385) & (0.536,0.857)\end{array}$
$1 \quad[0.413,0.933] \quad[0.037,0.512] \quad[0.000,0.313]$ $\begin{array}{llll}(0.405,0.946) & (0.022,0.521) & (0.000,0.324)\end{array}$

$2[0.000,0.400] \quad[0.256,0.824] \quad[0.001,0.380]$ $\begin{array}{llll}(0.000,0.412) & (0.245,0.833) & (0.000,0.393)\end{array}$

$3[0.000,0.354][0.013,0.491] \quad[0.414,0.913]$ $(0.000,0.365) \quad(0.003,0.501) \quad(0.404,0.926)$

II. With Shape Restrictions

A. Arbitrary, Independent Misclassification $(\mathrm{Q}=0.20)$

$$
\begin{array}{lll}
1 & 2 & 3
\end{array}
$$

$1 \quad[0.449,0.948] \quad[0.021,0.490] \quad[0.000,0.283]$ $\begin{array}{lll}(0.433,0.963) & (0.003,0.506) & (0.000,0.306)\end{array}$

$2[0.000,0.390] \quad[0.273,0.815] \quad[0.009,0.362]$ $\begin{array}{lll}(0.000,0.409) & (0.257,0.831) & (0.000,0.381)\end{array}$

$3[0.000,0.340] \quad[0.001,0.460] \quad[0.462,0.889]$ $(0.000,0.351) \quad(0.000,0.473) \quad(0.448,0.914)$

B. Uniform, Independent Misclassification $(\mathrm{Q}=0.20)$

$1 \quad[0.539,0.855] \quad[0.080,0.400] \quad[0.000,0.152]$ $\begin{array}{lll}(0.524,0.871) & (0.064,0.415) \quad(0.000,0.184)\end{array}$

$2[0.058,0.333] \quad[0.370,0.715] \quad[0.075,0.305]$ $\begin{array}{llll}(0.045,0.350) & (0.354,0.730) & (0.062,0.322)\end{array}$

$3[0.000,0.223][0.056,0.373] \quad[0.549,0.832]$ $(0.000,0.244) \quad(0.040,0.385) \quad(0.536,0.857)$

\section{1}

$1 \quad[0.506,0.872] \quad[0.096,0.419] \quad[0.000,0.180]$ $\begin{array}{lll}(0.498,0.882) & (0.082,0.427) \quad(0.000,0.196)\end{array}$

$2[0.041,0.342] \quad[0.356,0.722] \quad[0.067,0.322]$ $\begin{array}{lll}(0.034,0.354) & (0.346,0.732) & (0.057,0.335)\end{array}$

$3[0.000,0.239][0.075,0.399] \quad[0.515,0.822]$ $(0.000,0.254) \quad(0.063,0.409) \quad(0.505,0.837)$
$1[0.454,0.890] \quad[0.063,0.478] \quad[0.000,0.287]$ $\begin{array}{llll}(0.437,0.907) & (0.048,0.493) & (0.000,0.308)\end{array}$

$2[0.000,0.394] \quad[0.283,0.808] \quad[0.024,0.372]$ $\begin{array}{lll}(0.000,0.407) & (0.271,0.820) & (0.013,0.386)\end{array}$

$3[0.000,0.340] \quad[0.007,0.478] \quad[0.435,0.917]$ $\begin{array}{lll}(0.000,0.356) & (0.000,0.486) \quad(0.425,0.932)\end{array}$

$1 \quad[0.538,0.810] \quad[0.126,0.394] \quad[0.000,0.164]$ $\begin{array}{llll}(0.522,0.828) & (0.111,0.408) & (0.000,0.194)\end{array}$

$2[0.061,0.336] \quad[0.379,0.708] \quad[0.092,0.315]$ $\begin{array}{lll}(0.051,0.346) & (0.367,0.718) & (0.080,0.328)\end{array}$

$3[0.000,0.213][0.080,0.389] \quad[0.533,0.821]$ $(0.000,0.230) \quad(0.068,0.398) \quad(0.522,0.838)$

$1 \quad[0.413,0.933] \quad[0.037,0.512] \quad[0.000,0.313]$ $\begin{array}{lll}(0.405,0.946) & (0.022,0.521) & (0.000,0.324)\end{array}$

$2[0.000,0.400] \quad[0.256,0.824] \quad[0.001,0.380]$ $\begin{array}{lll}(0.000,0.412) & (0.245,0.833) \quad(0.000,0.393)\end{array}$

$3 \quad[0.000,0.354][0.013,0.491] \quad[0.414,0.913]$ $(0.000,0.365) \quad(0.003,0.501) \quad(0.404,0.926)$

$1 \quad[0.454,0.890] \quad[0.063,0.478] \quad[0.000,0.287]$ $\begin{array}{lll}(0.437,0.907) & (0.048,0.493) & (0.000,0.308)\end{array}$

$2[0.000,0.394] \quad[0.283,0.808] \quad[0.024,0.372]$ $\begin{array}{lll}(0.000,0.407) & (0.271,0.820) & (0.013,0.385)\end{array}$

$3 \quad[0.000,0.340] \quad[0.007,0.478] \quad[0.435,0.917]$ $\begin{array}{lll}(0.000,0.355) & (0.000,0.486) & (0.425,0.932)\end{array}$
$1[0.506,0.872] \quad[0.096,0.419] \quad[0.000,0.180]$ $(0.498,0.882) \quad(0.082,0.427) \quad(0.000,0.196)$

$2[0.041,0.342] \quad[0.356,0.722] \quad[0.067,0.322]$ $\begin{array}{lll}(0.034,0.354) \quad(0.346,0.732) & (0.057,0.335)\end{array}$

$3[0.000,0.239][0.075,0.399] \quad[0.515,0.822]$
1

$1[0.538,0.810][0.126,0.394]$ [0.000,0.164] $\begin{array}{llll}(0.522,0.828) & (0.111,0.408) & (0.000,0.194)\end{array}$

$2 \quad[0.061,0.336] \quad[0.379,0.708] \quad[0.092,0.311]$ $\begin{array}{llll}(0.051,0.346) & (0.367,0.718) & (0.080,0.324)\end{array}$

$3[0.000,0.213] \quad[0.080,0.389] \quad[0.533,0.821]$ $\begin{array}{lll}(0.000,0.230) \quad(0.068,0.398) & (0.522,0.838)\end{array}$ 
Table E4. Tercile Transition Matrices: Summary Mobility Measures.

2004-2008 Panel

I. Expected Exit Time: Q1
CPME
AM
$\mathrm{UM}$
LSR + Shape + AIM
LSR + Shape + UIM
$\mathrm{M}+\mathrm{LSR}+$ Shape + AIM
$\mathrm{M}+\mathrm{LSR}+$ Shape + UIM
$[3.085,3.339]$
$[1.609,396.539]$
$[1.918,9.781]$
$[1.764,63.573]$
$[2.100,8.758]$
[1.764,26.880]
[2.100,7.750]

II. Expected Exit Time: Q3 CPME

[3.128,3.366]

AM

$\mathrm{UM}$

LSR + Shape + AIM

LSR + Shape + UIM

M + LSR + Shape + AIM

M + LSR + Shape + UIM

III. Upward Mobility

$\begin{array}{ll}\text { CPME } & {[0.449,0.486]} \\ \text { AM } & {[0.004,0.932]} \\ \text { UM } & {[0.153,0.782]} \\ \text { LSR + Shape + AIM } & {[0.024,0.851]} \\ \text { LSR + Shape + UIM } & {[0.171,0.714]} \\ \text { M + LSR + Shape + AIM } & {[0.056,0.851]} \\ \text { M + LSR + Shape + UIM } & {[0.194,0.714]}\end{array}$

IV. Downward Mobility

CPME
AM
UM
LSR + Shape + AIM
LSR + Shape + UIM
M + LSR + Shape + AIM
M + LSR + Shape + UIM

$[1.620,2783.745]$

[1.933,10.041]

[1.811,95.001]

[2.154,9.371]

[1.811,11.690]

[2.154,6.988]

[0.449,0.486]

[0.153,0.782]

$[0.024,0.851]$

$[0.056,0.851]$

$[0.446,0.480]$

[0.001,0.926]

$[0.149,0.776]$

[0.016,0.828]

[0.160,0.696]

$[0.128,0.828]$

$[0.215,0.696]$
2008-2012 Panel

I. Expected Exit Time: Q1

CPME

AM

[3.117,3.275]

$[1.615,141.850]$

[1.926,9.341]

[1.680,53.118]

[1.991,8.469]

$[1.680,18.456]$

[1.991,8.469]

II. Expected Exit Time: Q3

CPME

[3.064,3.234]

AM

[1.601,90.336]

[1.907,9.003]

$\mathrm{LSR}+$ Shape + AIM

$[1.677,21.409]$

LSR + Shape + UIM

$[2.019,7.143]$

$\mathrm{M}+$ LSR + Shape + AIM

$[1.677,13.438]$

M + LSR + Shape + UIM

$[2.019,6.119]$

III. Upward Mobility

CPME

$[0.458,0.481]$

AM

[0.011,0.929]

$\mathrm{UM}$

[0.161,0.779]

LSR + Shape + AIM

$[0.028,0.893]$

LSR + Shape + UIM

$[0.177,0.753]$

[0.081,0.893]

$[0.177,0.753]$

M + LSR + Shape + UIM

$[0.464,0.490]$

$[0.017,0.937]$

[0.167,0.787]

[0.070,0.894]

$[0.210,0.743]$

[0.112,0.894]

$[0.245,0.743]$

Notes: Outcome $=$ modified OECD equivalized income. CPME $=$ classification-preserving measurement error. $\mathrm{AM}=$ arbitrary misclassification. $\mathrm{UM}=$ uniform misclassification. $\mathrm{I}$ = independence. $\mathrm{LSR}=$ level set restrictions. $\mathrm{M}=$ monotonicity. $90 \%$ confidence intervals for bounds provided in brackets based on estimates in Tables E1-E3. See text for further details. 
Table E5. Tercile Transition Matrices: Misclassification Assumptions.

\section{4-2008 Panel}

I. Classification-Preserving Measurement Error

2

$1 \quad[0.689,0.689] \quad[0.244,0.244] \quad[0.068,0.068]$ $\begin{array}{lll}(0.677,0.701) & (0.232,0.255) \quad(0.061,0.075)\end{array}$

$2 \quad[0.221,0.221] \quad[0.550,0.550] \quad[0.229,0.229]$ $\begin{array}{lll}(0.209,0.233) & (0.536,0.563) & (0.218,0.240)\end{array}$

$3 \quad[0.090,0.090] \quad[0.207,0.207] \quad[0.703,0.703]$ $(0.082,0.099) \quad(0.196,0.218) \quad(0.692,0.714)$

II. Arbitrary Misclassification $(\mathrm{Q}=0.20)$

$$
2
$$

$1 \quad[0.389,0.989] \quad[0.000,0.544] \quad[0.000,0.368]$ $\begin{array}{llll}(0.379,0.997) & (0.000,0.553) & (0.000,0.374)\end{array}$

$2[0.000,0.521] \quad[0.250,0.850] \quad[0.000,0.529]$ $\begin{array}{llll}(0.000,0.531) & (0.239,0.860) & (0.000,0.538)\end{array}$

$3 \quad[0.000,0.390] \quad[0.000,0.507] \quad[0.403,1.000]$ $\begin{array}{lll}(0.000,0.397) & (0.000,0.515) \quad(0.394,1.000)\end{array}$

III. Uniform Misclassification $(\mathrm{Q}=0.20)$

3

$1 \quad[0.489,0.889] \quad[0.044,0.444] \quad[0.000,0.268]$ $\begin{array}{lll}(0.479,0.898) & (0.034,0.453) & (0.000,0.274)\end{array}$

$2[0.021,0.421] \quad[0.350,0.750] \quad[0.029,0.429]$ $\begin{array}{llll}(0.012,0.431) & (0.339,0.760) & (0.020,0.438)\end{array}$

$3 \quad[0.000,0.290] \quad[0.007,0.407] \quad[0.503,0.903]$ $(0.000,0.297) \quad(0.000,0.415) \quad(0.494,0.911)$ $(0.678,0.692)$

$2[0.216,0.216] \quad[0.535,0.535] \quad[0.248,0.248]$



$3[0.099,0.099] \quad[0.222,0.222] \quad[0.679,0.679]$ $(0.093,0.104) \quad(0.214,0.231) \quad(0.670,0.688)$

1

$1 \quad[0.385,0.985] \quad[0.000,0.542] \quad[0.000,0.373]$ $\begin{array}{llll}(0.380,0.990) & (0.000,0.548) & (0.000,0.377)\end{array}$

$2[0.000,0.516] \quad[0.235,0.835] \quad[0.000,0.548]$ $\begin{array}{llll}(0.000,0.522) & (0.228,0.842) & (0.000,0.555)\end{array}$

$3 \quad[0.000,0.399][0.000,0.522] \quad[0.379,0.979]$ $(0.000,0.403) \quad(0.000,0.529) \quad(0.372,0.986)$
$1 \quad[0.485,0.885] \quad[0.042,0.442] \quad[0.000,0.273]$ $\begin{array}{llll}(0.480,0.890) & (0.036,0.448) & (0.000,0.277)\end{array}$

$2[0.016,0.416] \quad[0.335,0.735] \quad[0.048,0.448]$ $\begin{array}{lll}(0.011,0.422) & (0.328,0.742) & (0.042,0.455)\end{array}$

$3 \quad[0.000,0.299] \quad[0.022,0.422] \quad[0.479,0.879]$ \\ Pooled Panels}

$\left[\begin{array}{lll}0.689,0.689] & {[0.241,0.241]} & {[0.070,0.070]}\end{array}\right.$ $\begin{array}{lll}(0.682,0.696) & (0.235,0.248) \quad(0.065,0.075)\end{array}$

$2 \quad[0.217,0.217] \quad[0.540,0.540] \quad[0.243,0.243]$ $\begin{array}{lll}(0.211,0.224) & (0.532,0.547) \quad(0.237,0.248)\end{array}$

$3[0.094,0.094] \quad[0.219,0.219] \quad[0.687,0.687]$ $(0.089,0.098) \quad(0.213,0.225) \quad(0.681,0.693)$

$1 \quad[0.389,0.989] \quad[0.000,0.541] \quad[0.000,0.370]$ $\begin{array}{lll}(0.384,0.994) & (0.000,0.546) & (0.000,0.374)\end{array}$

$2[0.000,0.517] \quad[0.240,0.840] \quad[0.000,0.543]$ $\begin{array}{lll}(0.000,0.522) & (0.234,0.846) & (0.000,0.547)\end{array}$

$3 \quad[0.000,0.394] \quad[0.000,0.519] \quad[0.387,0.987]$ $\begin{array}{lll}(0.000,0.397) \quad(0.000,0.524) & (0.382,0.992)\end{array}$

$$
1
$$

2

$1[0.489,0.889] \quad[0.041,0.441] \quad[0.000,0.270]$ $\begin{array}{llll}(0.484,0.894) & (0.036,0.446) & (0.000,0.274)\end{array}$

$2[0.017,0.417] \quad[0.340,0.740] \quad[0.043,0.443]$ $\begin{array}{llll}(0.012,0.422) & (0.334,0.746) & (0.038,0.447)\end{array}$

$3[0.000,0.294][0.019,0.419] \quad[0.487,0.887]$

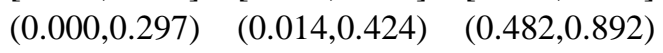

Notes: Outcome = per capita income. Point estimates for bounds provided in brackets obtained using 100 subsamples of size N/2 for bias correction. 90\% Imbens-Manski confidence intervals for the bounds provided in parentheses underneath point estimates obtained using 250 subsamples of size N/2. See text for further details. 
I. No Shape Restrictions

A. Arbitrary, Independent Misclassification $(Q=0.20)$$$
1
$$

2

3

1

2

3

$1 \quad[0.451,0.958] \quad[0.000,0.495] \quad[0.000,0.323]$ $\begin{array}{lll}(0.440,0.973) & (0.000,0.506) & (0.000,0.331)\end{array}$

$2[0.000,0.489] \quad[0.293,0.833] \quad[0.000,0.483]$ $\begin{array}{lll}(0.000,0.503) & (0.275,0.850) \quad(0.000,0.498)\end{array}$

$3[0.000,0.348][0.000,0.445] \quad[0.479,0.962]$ $\begin{array}{lll}(0.000,0.359) & (0.000,0.459) & (0.464,0.976)\end{array}$

B. Uniform, Independent Misclassification $(Q=0.20)$

$$
1
$$

$$
2
$$$$
3
$$

$1 \quad[0.541,0.869] \quad[0.069,0.405]$ [0.000,0.234] $\begin{array}{llll}(0.530,0.883) & (0.055,0.417) & (0.000,0.241)\end{array}$

$2[0.033,0.397] \quad[0.392,0.732] \quad[0.055,0.386]$ $\begin{array}{lll}(0.020,0.411) & (0.375,0.749) & (0.041,0.400)\end{array}$

$3[0.000,0.254][0.032,0.355] \quad[0.569,0.867]$ $(0.000,0.266) \quad(0.019,0.368) \quad(0.555,0.884)$

II. With Shape Restrictions

A. Arbitrary, Independent Misclassification $(Q=0.20)$

$$
1
$$

$$
2
$$

3

$1 \quad[0.451,0.958] \quad[0.000,0.495] \quad[0.000,0.323]$ $\begin{array}{lll}(0.440,0.973) & (0.000,0.506) & (0.000,0.331)\end{array}$

$2[0.000,0.489] \quad[0.293,0.833]$ [0.000,0.483] $\begin{array}{lll}(0.000,0.503) & (0.275,0.850) & (0.000,0.498)\end{array}$

$3 \quad[0.000,0.348] \quad[0.000,0.445] \quad[0.479,0.962]$ $\begin{array}{lll}(0.000,0.359) & (0.000,0.459) & (0.464,0.976)\end{array}$
$1[0.453,0.975] \quad[0.000,0.490] \quad[0.000,0.327]$ $\begin{array}{lll}(0.445,0.985) & (0.000,0.498) & (0.000,0.332)\end{array}$

$2[0.000,0.480] \quad[0.264,0.826] \quad[0.000,0.535]$ $\begin{array}{lll}(0.000,0.489) & (0.252,0.836) & (0.000,0.544)\end{array}$

$3[0.000,0.375] \quad[0.000,0.482] \quad[0.414,0.946]$ $\begin{array}{lll}(0.000,0.382) & (0.000,0.493) \quad(0.402,0.955)\end{array}$

\section{$10+\frac{10}{10}$}

$1[0.544,0.864] \quad[0.049,0.400] \quad[0.000,0.236]$ $\begin{array}{llll}(0.536,0.874) & (0.040,0.408) & (0.000,0.242)\end{array}$

$2[0.024,0.390] \quad[0.361,0.727] \quad[0.069,0.430]$ $\begin{array}{lll}(0.014,0.399) & (0.349,0.736) & (0.060,0.438)\end{array}$

$3[0.000,0.282][0.044,0.386] \quad[0.509,0.858]$ $\begin{array}{lll}(0.000,0.289) & (0.035,0.397) \quad(0.498,0.868)\end{array}$
$1 \quad[0.453,0.975] \quad[0.000,0.490] \quad[0.000,0.327]$ $\begin{array}{lll}(0.445,0.985) & (0.000,0.498) & (0.000,0.332)\end{array}$

$2[0.000,0.480] \quad[0.264,0.826] \quad[0.000,0.480]$ $\begin{array}{llll}(0.000,0.489) & (0.252,0.836) & (0.000,0.489)\end{array}$

$3[0.000,0.375][0.000,0.482] \quad[0.414,0.946]$ $\begin{array}{lll}(0.000,0.382) & (0.000,0.493) & (0.402,0.955)\end{array}$
10.467 .0

$1 \quad[0.467,0.920] \quad[0.021,0.474] \quad[0.000,0.313]$ $\begin{array}{llll}(0.457,0.934) & (0.011,0.484) \quad(0.000,0.320)\end{array}$

$2[0.000,0.461] \quad[0.285,0.819] \quad[0.011,0.507]$ $\begin{array}{lll}(0.000,0.471) & (0.272,0.832) & (0.000,0.517)\end{array}$

$3[0.000,0.362] \quad[0.001,0.464] \quad[0.438,0.938]$ $\begin{array}{lll}(0.000,0.367) & (0.000,0.474) \quad(0.428,0.946)\end{array}$

B. Uniform, Independent Misclassification $(\mathrm{Q}=0.20)$

$1 \quad[0.541,0.869] \quad[0.069,0.405] \quad[0.000,0.234]$ $\begin{array}{lll}(0.530,0.883) & (0.055,0.417) & (0.000,0.241)\end{array}$

$2 \quad[0.033,0.397] \quad[0.392,0.732] \quad[0.055,0.386]$ $\begin{array}{llll}(0.020,0.411) & (0.375,0.749) & (0.041,0.400)\end{array}$

$3 \quad[0.000,0.254] \quad[0.032,0.355] \quad[0.569,0.867]$ $\begin{array}{lll}(0.000,0.266) \quad(0.019,0.368) & (0.555,0.884)\end{array}$

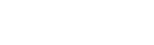

$1 \quad[0.556,0.835] \quad[0.100,0.386] \quad[0.000,0.228]$ $\begin{array}{llll}(0.546,0.847) & (0.088,0.395) & (0.000,0.234)\end{array}$

$2[0.037,0.373] \quad[0.381,0.724] \quad[0.090,0.410]$ $\begin{array}{llll}(0.027,0.383) & (0.368,0.735) & (0.077,0.420)\end{array}$

$3[0.000,0.272] \quad[0.053,0.373] \quad[0.530,0.851]$

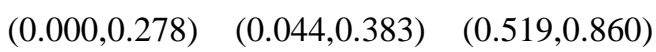

$\begin{array}{llll}(0.000,0.289) & (0.035,0.397) & (0.498,0.868)\end{array}$

Notes: Outcome = per capita income. Point estimates for bounds provided in brackets obtained using 100 subsamples of size N/2 for bias provided in parentheses underneath point estimates obtained using 250 subsamples of size N/2. See Table 3 and text for further details. 
Table E7. Tercile Transition Matrices: Monotonicity + Level Set Restrictions.

I. No Shape Restrictions

A. Arbitrary, Independent Misclassification $(Q=0.20)$$$
1
$$

2

3

1

2

3

$1 \quad[0.451,0.934] \quad[0.036,0.495] \quad[0.000,0.311]$ $\begin{array}{llll}(0.440,0.951) & (0.017,0.506) & (0.000,0.330)\end{array}$

$2 \quad[0.003,0.387] \quad[0.304,0.818] \quad[0.010,0.368]$ $\begin{array}{lll}(0.000,0.402) & (0.288,0.833) & (0.000,0.389)\end{array}$

$3 \quad[0.000,0.343] \quad[0.003,0.445] \quad[0.479,0.928]$ $\begin{array}{lll}(0.000,0.354) & (0.000,0.459) \quad(0.464,0.949)\end{array}$

$1 \quad[0.453,0.936] \quad[0.011,0.490] \quad[0.000,0.318]$ $\begin{array}{lll}(0.445,0.949) & (0.000,0.498) & (0.000,0.325)\end{array}$

$2[0.002,0.370] \quad[0.271,0.823] \quad[0.011,0.426]$ $\begin{array}{llll}(0.000,0.381) & (0.260,0.833) & (0.003,0.439)\end{array}$

$3[0.000,0.335] \quad[0.029,0.482] \quad[0.414,0.899]$ $(0.000,0.345) \quad(0.015,0.493) \quad(0.402,0.913)$

B. Uniform, Independent Misclassification $(\mathrm{Q}=0.20)$

$$
1
$$$$
2
$$$$
3
$$

\section{1}

$1 \quad[0.541,0.847][0.095,0.405] \quad[0.000,0.172]$ $\begin{array}{lll}(0.530,0.864) & (0.078,0.417) & (0.000,0.200)\end{array}$

$2[0.054,0.330] \quad[0.403,0.716] \quad[0.071,0.312]$ $\begin{array}{lll}(0.036,0.345) & (0.387,0.731) & (0.058,0.332)\end{array}$

$3[0.000,0.254][0.067,0.355] \quad[0.569,0.820]$ $(0.000,0.266) \quad(0.047,0.368) \quad(0.555,0.846)$
$1 \quad[0.544,0.843] \quad[0.084,0.400] \quad[0.000,0.212]$ $\begin{array}{lll}(0.536,0.858) & (0.073,0.408) & (0.000,0.224)\end{array}$

$2[0.051,0.313] \quad[0.365,0.719] \quad[0.080,0.365]$ $\begin{array}{lll}(0.043,0.324) & (0.355,0.729) \quad(0.071,0.377)\end{array}$

$3[0.000,0.210][0.086,0.386] \quad[0.509,0.841]$ $\begin{array}{lll}(0.000,0.225) & (0.073,0.397) \quad(0.498,0.853)\end{array}$

II. With Shape Restrictions

A. Arbitrary, Independent Misclassification $(Q=0.20)$

$$
1
$$

2

3

$1 \quad[0.451,0.934] \quad[0.036,0.495] \quad[0.000,0.311]$ $\begin{array}{lll}(0.440,0.951) \quad(0.017,0.506) & (0.000,0.330)\end{array}$

$2[0.003,0.387] \quad[0.304,0.818] \quad[0.010,0.368]$ $\begin{array}{llll}(0.000,0.402) & (0.288,0.833) & (0.000,0.389)\end{array}$

$3 \quad[0.000,0.343] \quad[0.003,0.445] \quad[0.479,0.928]$ $\begin{array}{lll}(0.000,0.354) \quad(0.000,0.459) & (0.464,0.949)\end{array}$
1

$1 \quad[0.453,0.936] \quad[0.011,0.490] \quad[0.000,0.315]$ $\begin{array}{lll}(0.445,0.949) & (0.000,0.498) \quad(0.000,0.322)\end{array}$

$2[0.002,0.370] \quad[0.271,0.823] \quad[0.011,0.401]$ $\begin{array}{lll}(0.000,0.381) & (0.260,0.833) \quad(0.003,0.411)\end{array}$

$3[0.000,0.335] \quad[0.029,0.482] \quad[0.414,0.899]$ $(0.000,0.345) \quad(0.015,0.493) \quad(0.402,0.913)$

B. Uniform, Independent Misclassification $(\mathrm{Q}=0.20)$

$1 \quad[0.541,0.847] \quad[0.095,0.405] \quad[0.000,0.172]$ $\begin{array}{lll}(0.530,0.864) \quad(0.078,0.417) & (0.000,0.200)\end{array}$

$2[0.054,0.330] \quad[0.403,0.716] \quad[0.071,0.311]$ $\begin{array}{lll}(0.036,0.345) & (0.387,0.731) & (0.058,0.330)\end{array}$

$3[0.000,0.254][0.067,0.355] \quad[0.569,0.820]$ $(0.000,0.266) \quad(0.047,0.368) \quad(0.555,0.846)$
1

2

$1[0.544,0.843][0.084,0.400] \quad[0.000,0.212]$ $\begin{array}{lll}(0.536,0.858) & (0.073,0.408) \quad(0.000,0.224)\end{array}$

$2[0.051,0.313] \quad[0.365,0.719] \quad[0.080,0.326]$ $\begin{array}{lll}(0.043,0.324) & (0.355,0.729) & (0.071,0.337)\end{array}$

$3[0.000,0.210] \quad[0.086,0.386] \quad[0.509,0.841]$ $(0.000,0.225) \quad(0.073,0.397) \quad(0.498,0.853)$
$1 \quad[0.467,0.892] \quad[0.060,0.474] \quad[0.000,0.263]$ $\begin{array}{llll}(0.457,0.909) & (0.044,0.484) & (0.000,0.285)\end{array}$

$2[0.000,0.362] \quad[0.293,0.789] \quad[0.042,0.397]$ $\begin{array}{lll}(0.000,0.374) & (0.279,0.802) & (0.031,0.412)\end{array}$

$3[0.000,0.324][0.029,0.464] \quad[0.438,0.888]$ $\begin{array}{lll}(0.000,0.339) & (0.014,0.474) \quad(0.428,0.911)\end{array}$

$\begin{array}{lll}1 & 2 & 3\end{array}$

$1[0.556,0.807] \quad[0.131,0.386] \quad[0.000,0.132]$ $\begin{array}{lll}(0.546,0.825) & (0.114,0.395) & (0.000,0.162)\end{array}$

$2[0.064,0.304] \quad[0.388,0.691] \quad[0.105,0.333]$ $\begin{array}{llll}(0.054,0.317) & (0.376,0.704) & (0.094,0.348)\end{array}$

$3[0.000,0.192] \quad[0.083,0.373] \quad[0.530,0.792]$ $\begin{array}{lll}(0.000,0.209) \quad(0.068,0.383) & (0.519,0.817)\end{array}$

Notes: Outcome = per capita income. Point estimates for bounds provided in brackets obtained using 100 subsamples of size N/2 for bias correction. $90 \%$ Imbens-Manski confidence intervals for the bounds provided in parentheses underneath point estimates obtained using 250 subsamples of size N/2. See Table 4 and text for further details. 
Table E8. Tercile Transition Matrices: Summary Mobility Measures.

2004-2008 Panel

I. Expected Exit Time: Q1
CPME
AM
$\mathrm{UM}$
LSR + Shape + AIM
LSR + Shape + UIM
$\mathrm{M}+\mathrm{LSR}+$ Shape + AIM
$\mathrm{M}+\mathrm{LSR}+$ Shape + UIM
$[3.092,3.339]$
$[1.611,396.058]$
$[1.920,9.792]$
$[1.784,37.238]$
[2.126,8.533]
[1.784,20.419]
[2.126,7.341]

II. Expected Exit Time: Q3 CPME

[3.243,3.494]

$[1.650,$.

AM

$\mathrm{UM}$

LSR + Shape + AIM

LSR + Shape + UIM

$\mathrm{M}+\mathrm{LSR}+$ Shape + AIM

M + LSR + Shape + UIM

$1.977,11.277]$

[1.867,41.500]

[2.248,8.605]

$[1.867,19.575]$

[2.248,6.493]

III. Upward Mobility

$\begin{array}{ll}\text { CPME } & {[0.449,0.485]} \\ \text { AM } & {[0.004,0.931]} \\ \text { UM } & {[0.153,0.781]} \\ \text { LSR + Shape + AIM } & {[0.040,0.841]} \\ \text { LSR + Shape + UIM } & {[0.176,0.706]} \\ \text { M + LSR + Shape + AIM } & {[0.073,0.841]} \\ \text { M + LSR + Shape + UIM } & {[0.204,0.706]}\end{array}$

IV. Downward Mobility

$\begin{array}{ll}\text { CPME } & {[0.429,0.463]} \\ \text { AM } & {[0.000,0.909]} \\ \text { UM } & {[0.133,0.759]} \\ \text { LSR + Shape + AIM } & {[0.036,0.803]} \\ \text { LSR + Shape + UIM } & {[0.174,0.667]} \\ \text { M + LSR + Shape + AIM } & {[0.077,0.803]} \\ \text { M + LSR + Shape + UIM } & {[0.231,0.667]}\end{array}$

2008-2012 Panel

I. Expected Exit Time: Q1

CPME

AM

[3.107,3.246]

[1.612,104.290]

[1.922,9.125]

$[1.803,67.704]$

[2.154,7.924]

[1.803,19.763]

[2.154,7.039]

II. Expected Exit Time: Q3

CPME

[3.029,3.204]

AM

[1.592,70.962]

[1.893,8.765]

$\mathrm{LSR}+$ Shape + AIM

$[1.673,22.294]$

LSR + Shape + UIM

[1.991,7.560]

$\mathrm{M}+$ LSR + Shape + AIM

[1.673,11.502]

M + LSR + Shape + UIM

[1.991,6.819]

III. Upward Mobility

CPME

[0.462,0.483]

$\mathrm{AM}$

[0.014,0.930]

$\mathrm{UM}$

[0.164,0.780]

LSR + Shape + AIM

[0.022,0.832]

LSR + Shape + UIM

$[0.189,0.696]$

[0.076,0.832]

$[0.213,0.696]$

IV. Downward Mobility

CPME

[0.468,0.495]

AM

[0.021,0.942]

UM

[0.171,0.792]

LSR + Shape + AIM

$[0.067,0.896]$

LSR + Shape + UIM

[0.198,0.753]

M + LSR + Shape + AIM

$[0.130,0.896]$

M + LSR + Shape + UIM

$[0.220,0.753]$

Notes: Outcome $=$ per capita income. CPME $=$ classification-preserving measurement error. AM $=$ arbitrary misclassification. UM = uniform misclassification. I = independence. LSR = level set restrictions. $\mathrm{M}=$ monotonicity. $90 \%$ confidence intervals for bounds provided in brackets based on estimates in Tables E5-E7. See text for further details. 